\title{
Optical Excitation in Scanning Tunneling Microscopy: From Surface Photovoltages to Charge Dynamics on the Atomic Scale
}

\author{
Dissertation \\ zur Erlangung des mathematisch-naturwissenschaftlichen Doktorgrades \\ "Doctor rerum naturalium" \\ der Georg-August-Universität Göttingen \\ im Promotionsprogramm ProPhys \\ der Georg-August-University School of Science (GAUSS)
}

vorgelegt von

\section{Philipp Kloth}

aus Hildesheim

Göttingen

2016 

Betreuungsausschuss

Dr. Martin Wenderoth,

IV. Physikalisches Institut, Georg-August-Universität Göttingen

Prof. Dr. Claus Ropers,

IV. Physikalisches Institut, Georg-August-Universität Göttingen

Prof. Dr. Rainer G. Ulbrich,

IV. Physikalisches Institut, Georg-August-Universität Göttingen

Mitglieder der Prüfungskommission

Referent: Dr. Martin Wenderoth,

IV. Physikalisches Institut, Georg-August-Universität Göttingen

Korreferent: Prof. Dr. Stefan Mathias,

I. Physikalisches Institut, Georg-August-Universität Göttingen

Korreferent: Prof. Dr. Jascha Repp,

Institut für Experimentelle und Angewandte Physik, Universität Regensburg

Weitere Mitglieder der Prüfungskommission

Prof. Dr. Claus Ropers,

IV. Physikalisches Institut, Georg-August-Universität Göttingen

Prof. Dr. Christian Jooß,

Institut für Materialphysik, Georg-August-Universität Göttingen

Prof. Dr. Cynthia Volkert,

Institut für Materialphysik, Georg-August-Universität Göttingen

Prof. Dr. Hans Hofsäss,

II. Physikalisches Institut, Georg-August-Universität Göttingen

Tag der Disputation: 15.12.2016 



\section{Abstract}

In this thesis, the successful implementation of optical excitation for time-resolved Scanning Tunneling Microscopy (STM) is presented. The fruitful combination of these two experimental methods allows investigating photo-induced dynamic processes on the nanosecond time scale with atomic resolution. The optical setup provides a great versatility regarding the adjustment of excitation parameters such as optical pulse height, pulse width or pulse repetition rate to the experimental needs. Moreover, for the first time, it is possible to disentangle and quantify thermally induced effects, e.g. thermal expansion of the STM tip, from the originally inquired signals, resulting from photo-triggered charge dynamics at the sample surface.

Using continuous wave optical illumination at the Gallium-Arsenide(110) (GaAs) surface, it is proven in this thesis, that the presence of excited holes, accumulating in the tip-induced Space Charge Region (SCR) beneath the STM tip not only creates a Surface Photovoltage (SPV) but also opens an additional tunneling channel. Current dependent studies show that this extra tunneling process exhibits a high impact on the charge concentration at the surface. Assuming a steady state between charge generation via optical excitation and charge annihilation via the tunneling process, parameters such as the diffusional or field driven transport rate towards the surface are determined.

The build-up and relaxation of an SPV is connected to various microscopic processes, e.g., charge transport or carrier recombination. With pulsed optical excitation, these mechanisms can be probed and disentangled. Whereas for low tunnel rates the decay of the system after optical excitation is mainly determined by the charge annihilation via the tunneling process, for high rates the recharging of dopants becomes visible. Studies of this ionization process for donors, positioned at different depths beneath the surface, reveal a significant local inhomogeneity. By applying a field driven ionization mechanism, it turns out, that the dopants cannot be treated independently from each other. Instead, the ionization dynamics have to be treated as an interacting network of coupled, locally fixed and randomly distributed charge centers.

\section{Keywords}

Time-Resolved Scanning Tunneling Microscopy, Nanotechnology, Gallium-Arsenide (GaAs), Semiconductor Surfaces, Optical Excitation, Surface Photovoltage (SPV), Charge Transport, Donor Charging Dynamics 



\section{Introduction}

For semiconductor-based electronic devices, the accomplishment of nanometer-sized structures requests for a precise characterization of the electronic properties on the atomic scale ${ }^{1}$. Fig. i shows the 3D potential landscape of a field effect transistor in its classical source-gate-drain geometry,

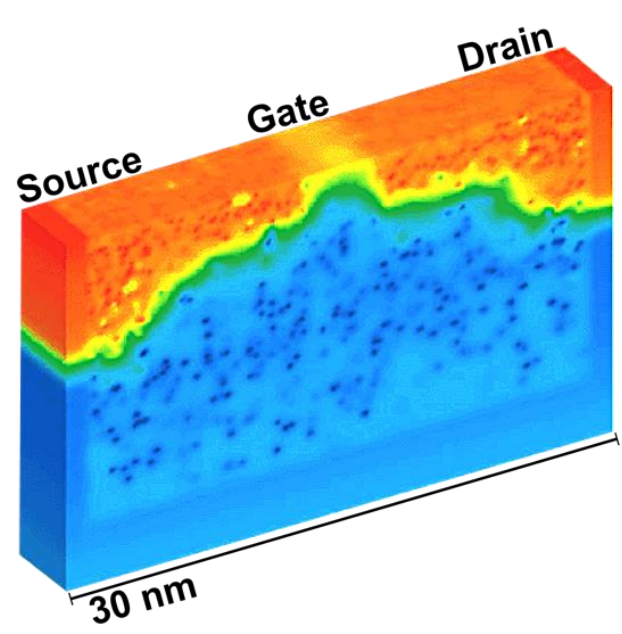

Fig. i 3D simulation of the potential landscape of a $30.30 \mathrm{~nm}^{2}$ large field effect transistor. Plotted in color code is the electric potential. Already at this length scale the discreetness of single doping atoms becomes visible. Picture taken from ${ }^{2}$. including randomly distributed doping atoms ${ }^{2}$. Though it still has a size of $30 \cdot 30 \mathrm{~nm}^{2}$, the discreteness of single dopants as localized charge centers already becomes visible. Further improvement of such structures demands for an exact understanding of structural and electronic properties on the smallest scale.

For a long period of time, the most popular method to characterize electronic properties of semiconductor materials was based on light interaction with the material. An important observable in this context is the so-called Surface Photovoltage (SPV). Firstly described by Brattain et $\mathrm{al}^{3}{ }^{3}$, it is defined as the change in surface potentials under optical illumination. Microscopically, the build-up of a SPV is connected to various processes, e.g., electron-hole generation, charge transfer, ionization/neutralization of local defects and intrinsic electron-hole pair recombination. With the help of the SPV it was possible to extract sample properties such as the carrier diffusion length or carrier mobility ${ }^{4,5}$. However, due to the diffraction limit of light, nanoscopic information are lacking.

An outstanding tool for determining the morphological but at the same time electronic properties on the atomic scale is the Scanning Tunneling Microscope (STM). In fact, the very first investigated sample system by the STM was a semiconductor surface, namely the $7 \times 7$ reconstructed Silicon(111) surface ${ }^{6}$. Since then, this method has proven its great versatility for classifying nanometer-sized heterostructures, quantum-dots or even single atom systems ${ }^{7-9}$.

Already shortly after the invention of the STM, several groups have invested substantial effort ${ }^{10-12}$ to combine this technique with simultaneous optical excitation (Fig. ii). Resolving the SPV on the atomic scale was firstly done by Hamers et al. ${ }^{10}$. Their results indicate a great inhomogeneity depending on the surface morphology of the silicon substrate. Other research groups followed this idea and performed local SPV studies on other substrates, as well as at single defects or small hetero/nanostructures ${ }^{13-16}$. 
A standard method employed in all these publications was the extraction of the SPV via comparing currentvoltage characteristics at different optical excitation intensities. However, other studies have shown that the tunnel current from the STM tip can have a significant influence on the SPV ${ }^{13,17,18}$. Especially at surfaces with a low surface charge density this can modify the local charge configuration considerably. A part of this thesis (chapter 3) focuses on the detailed study of the interplay between charge generation via continuous optical excitation and the charge annihilation via the tunnel current and its impact onto local fields - in this case the nanometersized tip-induced space charge region (SCR) at the GaAs(110) surface. For the first time, we were able to directly visualize the contribution of the photo-

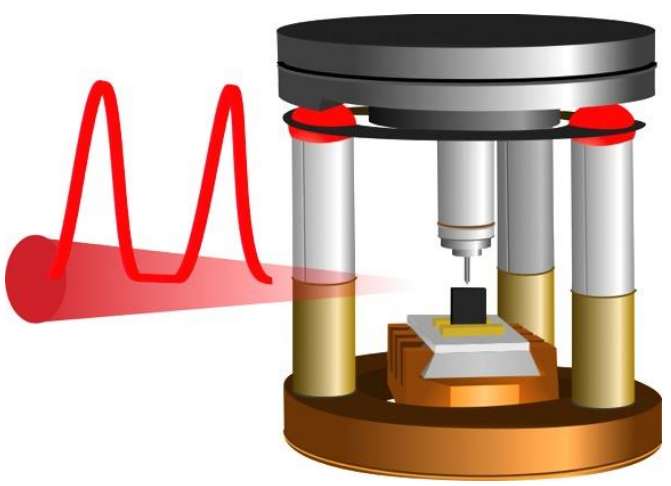

Fig. ii Sketch of optical excitation in Scanning Tunneling Microscopy. Pictured is an STM head (beetle design), used in our research group. Additional pulsed optical excitation at the tunnel junction triggers dynamic processes. A sophisticated pump-probe technique allows resolving these triggered processes on the atomic scale. generated charge in the tunneling process. Moreover, by tuning the tunnel current, the actual concentration of free charge carriers and thereby the size of the tip-induced SCR can be adjusted. Assuming a steady state between charge generation via optical excitation and charge annihilation via the tunnel process, the diffusion and drift rates of minority carriers have been extracted.

Surprisingly, a significant change of the current noise characteristics is found under optical excitation. Typically it is assumed that continuous charging or discharging of surface-near dopants, as already reported ${ }^{19,20}$, are responsible for this observation. However, a clarification of this assumption can only be given by accessing the time-resolved dynamics of the charge interplay at the surface.

Several research groups already attempted to complement the optical excitation with a pulsed operation in $\mathrm{STM}^{21-23}$ in order to investigate the dynamics of the excitation and relaxation processes. Focusing a pulsed laser beam onto the tunnel junction can induce major thermal issues, compromising a stable operation of the STM. In 2004, the research group of Hidemi Shigekawa from the University of Tsukuba presented an elegant modulation techniques of the optical pulses, which allowed to identify charge dynamics at the GaAs surface with an $\mathrm{STM}^{24}$.

Up to now, several improvements of their method enabled the investigation of this charge dynamics in a wide temporal range and also in the presence of local defects ${ }^{25}$. In a recent implementation the setup consists of two femtosecond laser oscillators and several high bandwidth Pockels cells for pulse picking. A major disadvantage for such a setup is the lack of flexibility in adjusting the excitation parameters to the needs of the experiment. A task of this thesis was to adapt 
a similar concept of the pulse generation but at the same time, to add a greater flexibility to the setup (chapter 2). By shaping a continuous wave, low noise laser beam into nanosecond pulses with the help of a high bandwidth electro-optical modulator and an arbitrary wave generator, excitation parameters like pulse amplitude, width or repetition cycle can be easily and seamlessly adjusted to the diverse experimental needs.

This new technique allows for investigating the carrier dynamics for the build-up and relaxation of the SPV in detail (chapter 4). Unlike previous observations ${ }^{25}$, significant dependencies between the relaxation dynamics, the set point current of the tunneling process and the applied laser intensity are observed. These results can be understood by different charge configurations at the surface for altering tunneling rates into the photo-generated charge. Whereas for low set point currents the optically induced signal is determined by the charge annihilation process via tunneling electrons, at higher currents the decay of the optically induced signal describes the recharging process of surface-near dopants. By acquiring spatiotemporally resolved maps of single donors, situated at different depths beneath the surface, we can elucidate this process in more detail. We conclude, that the charging process of donors cannot be treated as an isolated event. Instead, dopants have to be considered as an ensemble of stochastically distributed defects for which charging events are highly correlated to each other.

\section{Outline}

In chapter 1, a summary of models and notions, used in this thesis, is given.

Chapter 2 discusses the time-resolved STM technique. First, former implementations of this method from the literature are discussed. Subsequently, a detailed description of the setup, which has been developed within the scope of this work, is provided. A part of this chapter is currently under peer-review for publication.

In chapter 3 studies of optical effects at the GaAs(110) surface under continuous optical excitation are presented. Own studies demonstrate for the first time that photo-generated charge carriers contribute to the tunneling process in STM. This last part of the chapter is published in the scientific journal "Nature Communications".

Time-resolved measurements of the dynamics of photo-generated charge at the GaAs surface are presented in chapter 4. In the beginning of the chapter, a review on the current literature is given. Afterwards own studies are presented, demonstrating a significant inhomogeneity of charge dynamics on the atomic scale. This part of the chapter is under peer-review for publication.

Finally, an outlook for the time-resolved STM technique is presented in chapter 5. Possible further questions and possibilities at the GaAs surface are discussed. 
Please note, that the subchapters 2.2, 3.3 and 4.4 of this thesis are already published in scientific journals. At the beginning of each section, a small paragraph unravels the respective contributions of collaborations. Furthermore, for the sake of an increased readability of the thesis, each publication is summarized briefly. At the end of each chapter also a more general discussion is given. 


\section{Table of contents}

1 Scientific background and experimental methods ............................... 13

$1.1 \quad$ Scanning Tunneling Microscopy at the GaAs(110) surface ..................................................... 13

$1.2 \quad$ Surface Photovoltage and Scanning Tunneling Microscopy ....................................................... 18

2 Implementation of time resolution in STM techniques..................... 21

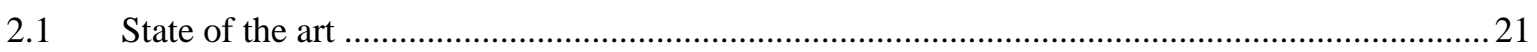

2.2 A versatile implementation of pulsed optical excitation in Scanning Tunneling Microscopy ....27

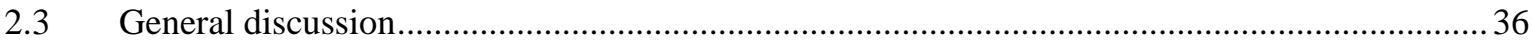

3 Optical excitation at semiconductor surfaces in STM experiments........ 39

3.1 How to extract the Surface Photovoltage in STM experiments ................................................... 39

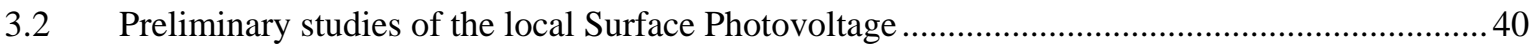

3.3 Controlling the screening process of a space charge region by minority carriers ........................ 43

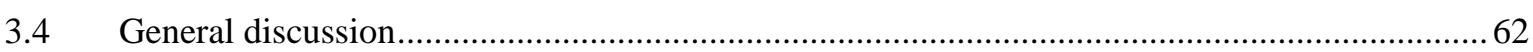

4 Pulsed optical excitation at the GaAs(110) surface ............................. 65

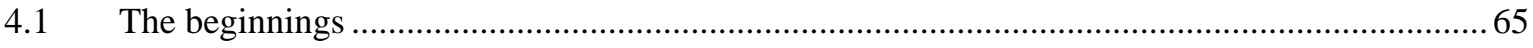

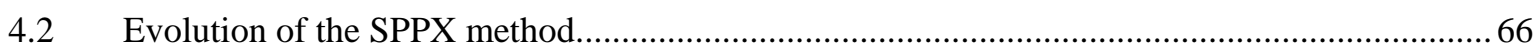

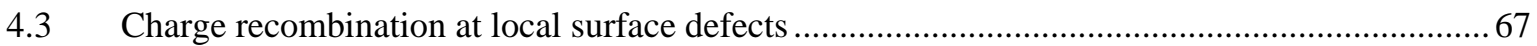

4.4 From time-resolved imaging of individual donors to their cooperative dynamics .......................70

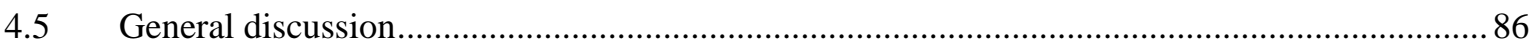

5 Outlook: The GaAs surface and its perspective ............................ 89

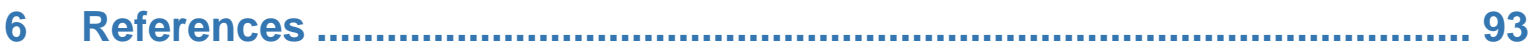





\section{Scientific background and experimental methods}

The following chapter gives a brief introduction to optical excitation in STM. It provides supplementary information on, e.g., the tunneling at the GaAs surface or the model of Surface Photovoltage (SPV), being important for the understanding of the subsequent chapters.

\subsection{Scanning Tunneling Microscopy at the GaAs(110) surface}

This subchapter presents an overview on Scanning Tunneling Microscopy at the GaAs(110) surface. It begins with a short summary on the sample/surface properties and the sample preparation method, used throughout this thesis. The following section is dedicated to the electrostatic interaction of the STM tip with the semiconductor surface, summarized as tip induced band bending (TIBB). A more general description on the STM method and details on the instrumentation and tip preparation were already described extensively elsewhere ${ }^{26-29}$. Parts of this chapter are adapted from ${ }^{26,27,29,30}$.

\section{Sample properties and sample preparation}

Gallium arsenide (GaAs) is a member of the group of III-V semiconductors. Consisting of Gallium and Arsenic, it crystallizes in the zinc-blende structure. An atomic sketch of the principle cleavage planes, namely the [110] surfaces, is shown in Fig. 1.1. Having the same amount of Gallium and Arsenic atoms, this surface carries no net charge. Due to the absence of bonding partners in this half space geometry, surface atoms relax ${ }^{31}$. This means that Gallium atoms are pulled down to their next nearest neighbors, whereas Arsenic atoms are tilted upwards.
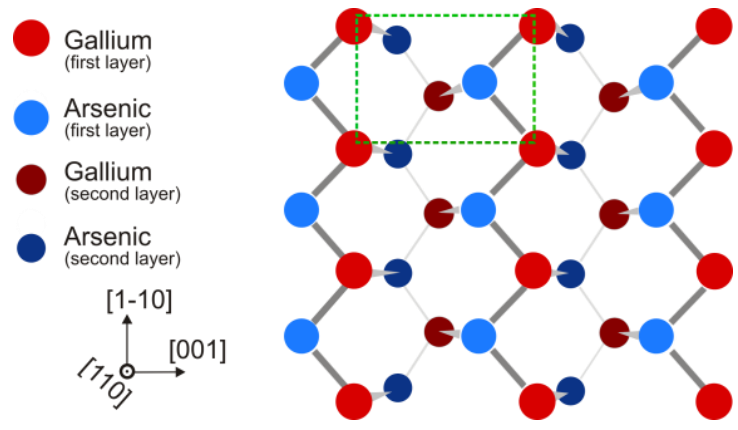

Fig. 1.1 Crystal structure at the GaAs(110) surface. Bulk GaAs is organized in the zinc-blende crystal structure. The top view on the $\mathrm{GaAs}(110)$ plane is shown. The green rectangle marks the surface unit cell. Adapted from ${ }^{27}$. 
Fig. 1.2 Resonant surface states at the $\mathrm{GaAs}(110)$ surface. Surface states can be directly mapped in STM topographies at different bias voltages, revealing different atomic corrugations. Taken from $^{26,34}$

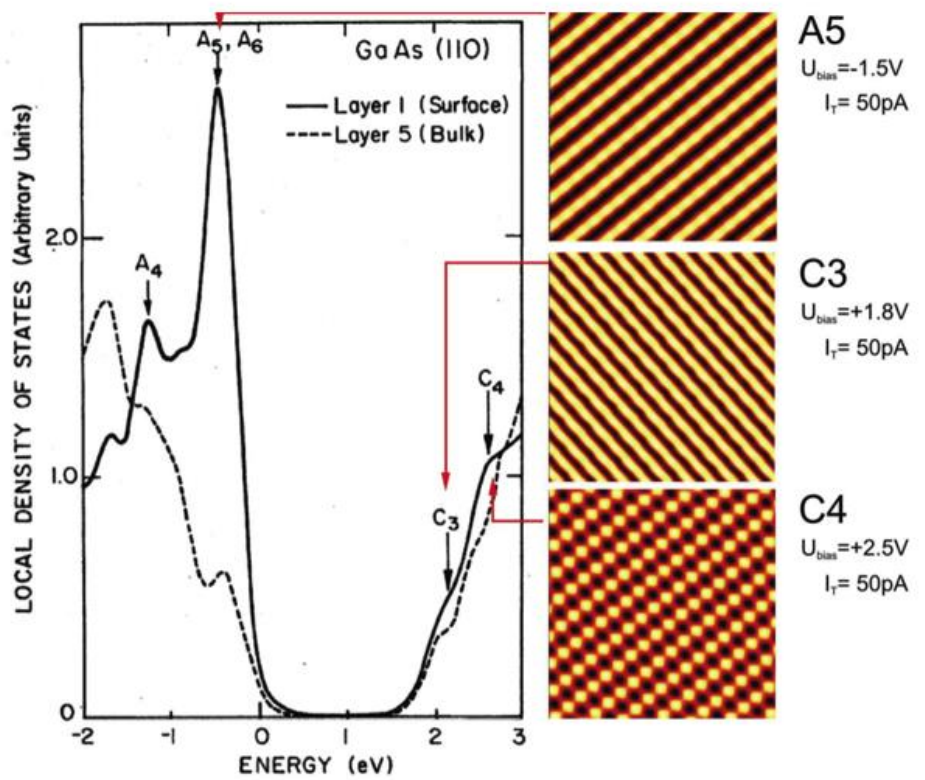

GaAs is a semiconductor with a direct band gap $\left(\mathrm{E}_{\text {gap }}=1.51 \mathrm{eV} \text { at } \mathrm{T}=77 \mathrm{~K}\right)^{32}$. Surface states for the (110)-surfaces appear as resonances in the valence and conduction band. A charge transfer during the surface relaxation results in filled states at the Arsenic atoms and empty states at the Gallium atoms ${ }^{33}$. Fig. 1.2 shows the calculated local density of states (LDoS) at the surface (solid) in comparison to the bulk LDoS ( $5^{\text {th }}$ layer). The surface resonances manifest as localized enhancements, energetically positioned near the band edges. These localized states at each atom, separately visible as different atomic corrugations at positive or negative bias voltages (Fig. 1.2), can be addressed in constant-current topographies. For positive sample voltages, empty states (conduction band) are addressed and the corrugation maxima are positioned at the dangling bonds above the Gallium atoms ( $\mathrm{C} 3$ and $\mathrm{C} 4$ resonant surface states). At negative voltages, the positions of the dangling bonds above the Arsenic atoms are mapped (A5 resonant surface states) ${ }^{33-35}$.

In order to obtain clean surfaces for STM investigations, GaAs is cleaved under ultra high vacuum (UHV) conditions. Starting with a commercial GaAs wafer having a doping density of $3 \cdot 10^{18} / \mathrm{cm}^{3}$ (n-doping with silicon), the preparation for the UHV cleavage process includes several previous processing steps. First, a $3.9 \mathrm{~mm}^{2}$ piece is cut from the wafer (Fig. 1.3a, b) and then thinned down to thicknesses of around $100 \mu \mathrm{m}$ by chemically etching in a bromine-ethanol solution.

To guarantee Ohmic contacts in STM experiments, metallic pads made of an alloy consisting of Gold and Germanium are evaporated at the [100] surface. The Gold serves as an inert capping layer. The Germanium, acting as doping atoms in GaAs, is used to generate a localized, highlydoped volume beneath the pad. This is achieved by annealing the GaAs sample under Nitrogen conditions up to $400{ }^{\circ} \mathrm{C}$ which leads to diffusion of Germanium into the surface. The resulting Ohmic resistance, measured from one pad to another, is in the range from 3 to $100 \Omega$. 
Fig. 1.3 Illustration of the sample preparation. (a) A $3.9 \mathrm{~mm}^{2}$ piece is cleaved from a [001] GaAs wafer. (b) After chemically etching to a thickness of $100 \mu \mathrm{m}$, Gold-Germanium pads were evaporated, working as Ohmic contacts. In the end, a $500 \mu \mathrm{m}$ long initial scratch, for the in-situ UHV cleavage process, is applied. Adapted from ${ }^{119}$.

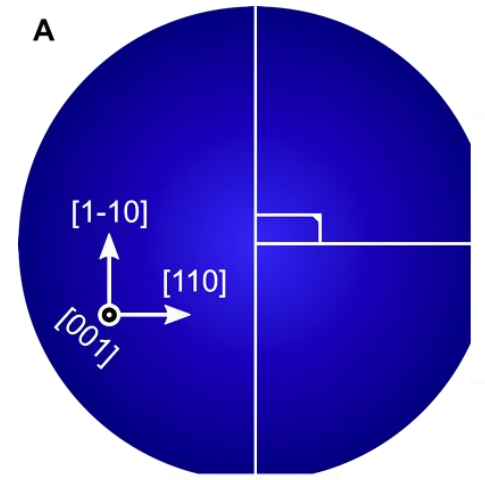

B

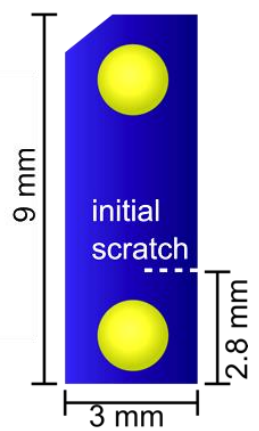

Finally, an initial scratch for the UHV cleavage process, is applied using a tungsten-carbide needle. This reduces the force needed to initialize the cleavage process, which promises an increased probability to generate defect free and large atomic flat terraces at the surface ${ }^{36,37}$. Also it defines the macroscopic height of the prepared GaAs surface, which is important for approaching the tip in STM experiments, having only a limited range of motion.

\section{Tip Induced Band Bending}

The induced potential of a metallic tip in tunnel contact (tip-sample distance $<1 \mathrm{~nm}$ ) at the GaAs surface is highly comparable to the system of a metal-insulator-semiconductor (MIS) junction, widely used, e.g., in field effect transistors. The potential drop between both subsystems results in a localized space charge region (SCR), which has considerable influence on the electronic properties of the system.

In standard STM theory, the sum of the applied bias voltage and the contact potential between tip and sample drops completely across the vacuum barrier. Free movable and high densities of charge carriers are assumed (e.g. metallic surfaces). Accordingly, the corresponding screening length of the tip potential inside the surface is small.

For semiconductor surfaces this assumption does not yield anymore. In comparison to metallic systems, locally fixed, ionized donors provide the charge, which screens the potential. Part of the potential leaks nanometers into the GaAs surface, forming a local SCR. Several parameters, e.g. doping density, potential between tip and sample and tip geometry, determine its shape and size. Fig. 1.4 shows the induced potential (color-coded) of a metallic tip, separated less than $1 \mathrm{~nm}$ from the sample, for several tip and sample parameters. The calculation is based on solving the Poisson equation (calculations were done with the commercial package COMSOL) in this half-space geometry $^{38}$. 


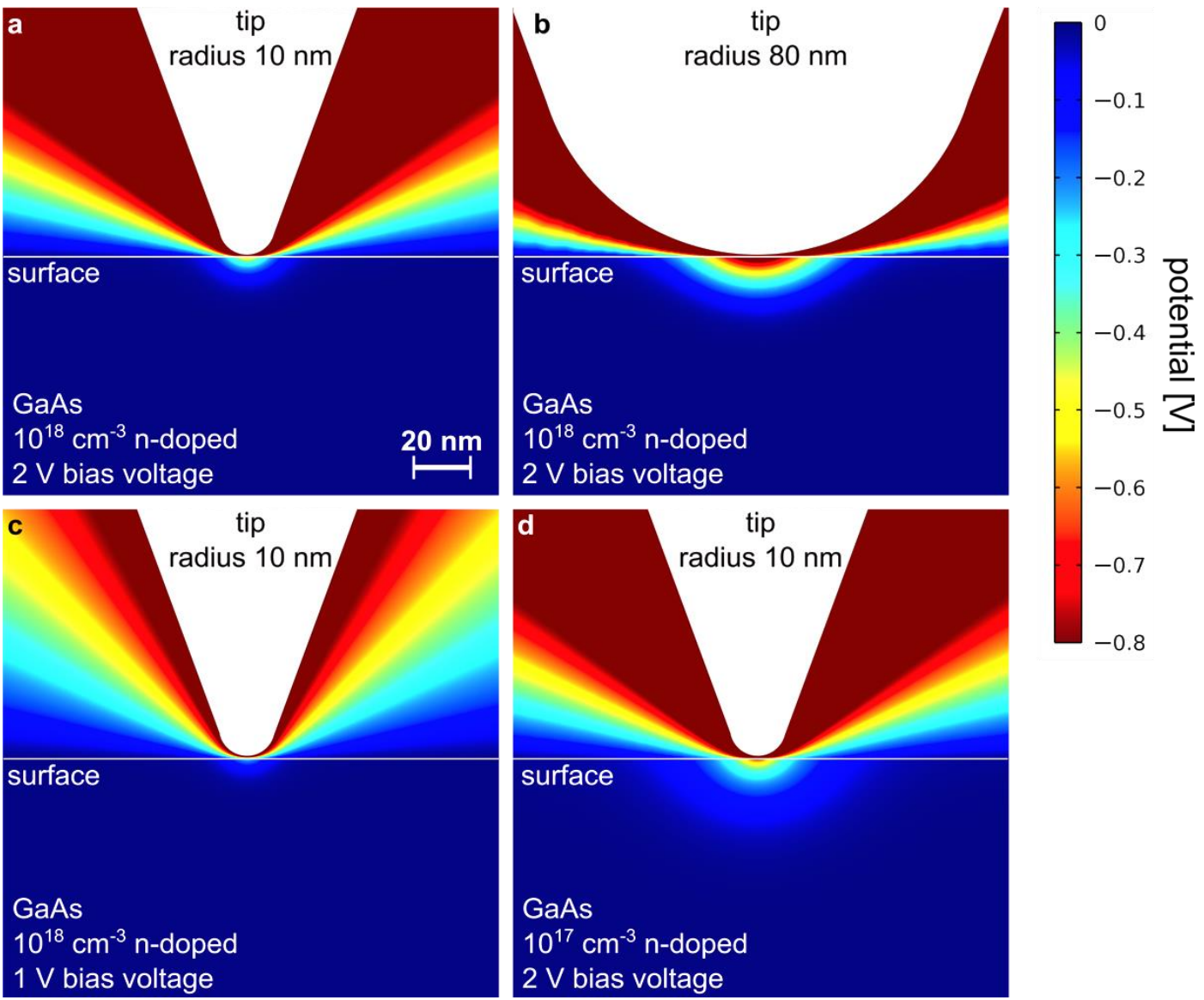

Fig. 1.4 Tip-induced potential at semiconductor surfaces with the STM tip in tunnel contact. The spatial extent of the induced depletion layer is very sensitive to the geometry of the STM tip, the doping density of the semiconductor and the potential between tip and sample. (a) Tip radius $10 \mathrm{~nm}$, doping $3 \cdot 10^{18} \mathrm{~cm}^{-3}, 2 \mathrm{~V}$ bias voltage. (b) Tip radius $80 \mathrm{~nm}$, doping $3 \cdot 10^{18} \mathrm{~cm}^{-3}, 2 \mathrm{~V}$ bias voltage. (c) Tip radius $10 \mathrm{~nm}$, doping $3 \cdot 10^{18} \mathrm{~cm}^{-3}, 1 \mathrm{~V}$ bias voltage. (d) Tip radius $10 \mathrm{~nm}$, doping $3 \cdot 10^{17} \mathrm{~cm}^{-3}, 2 \mathrm{~V}$ bias voltage. The color scale is cut at $-0.8 \mathrm{~V}$. Simulations: Courtesy of Dr. Tim Iffländer.

By defining a cut-off potential at $6 \mathrm{mV}(6 \mathrm{meV}$ : binding energy of electrons at bulk donor levels), the spatial extensions of the potential for each set of parameters is estimated. At a bias voltage of $2 \mathrm{~V}, 10 \mathrm{~nm}$ tip radius and a doping concentration of $3 \cdot 10^{18} \mathrm{~cm}^{-3}$ (Fig. 1.4a) the potential penetrates radially symmetric $12 \mathrm{~nm}$ into the surface. For this doping concentration, six dopants are positioned inside the SCR. By increasing the tip radius to $80 \mathrm{~nm}$ (Fig. 1.4b), the spatial extension of the SCR grows considerably, reaching now $30 \mathrm{~nm}$ into the sample. The total number of dopants rises nearly tenfold (55 ionized dopants). For a decrease of the potential between tip and sample (Fig. 1.4c, $1 \mathrm{~V}$ bias voltage, other parameters identical) the SCR shrinks. In this case it penetrates only $6 \mathrm{~nm}$ into the sample and only one ionized dopant is involved in the screening process. For a decrease of the doping density at a bias voltage of $2 \mathrm{~V}$ (Fig. 1.4d, $3 \cdot 10^{17} \mathrm{~cm}^{-3}$ ), the spatial extension of the potential run is increased as well (20 nm into the surface). However, analog to Fig. 1.4a the same amount of ionized dopants are positioned inside the SCR. These simulations give a good intuition for the SCR under different tunneling conditions. In this thesis, similar configurations as pictured in Fig. 1.4a (chapter 3) and Fig. 1.4c (chapter 4) are treated. 
In the rigid band model the influence of a surface potential is described by an energetic shift of the electronic states inside the semiconductor. In the context of STM experiments this method is summarized in the concepts of the tip-induced band bending (TIBB) ${ }^{39,40}$. Tunneling band schemes including TIBB effects are sketched in Fig. 1.5 for zero voltage and with tip and sample macroscopically separated (a), for positive (b) and negative (c) bias voltages (n-doped material). At zero voltage and with tip and sample isolated (Fig. 1.5a), both systems are in equilibrium. At the GaAs side, conduction band (CB) and valence band (VB) are undisturbed. The donor levels are positioned close to the conduction band edge (blue circles).

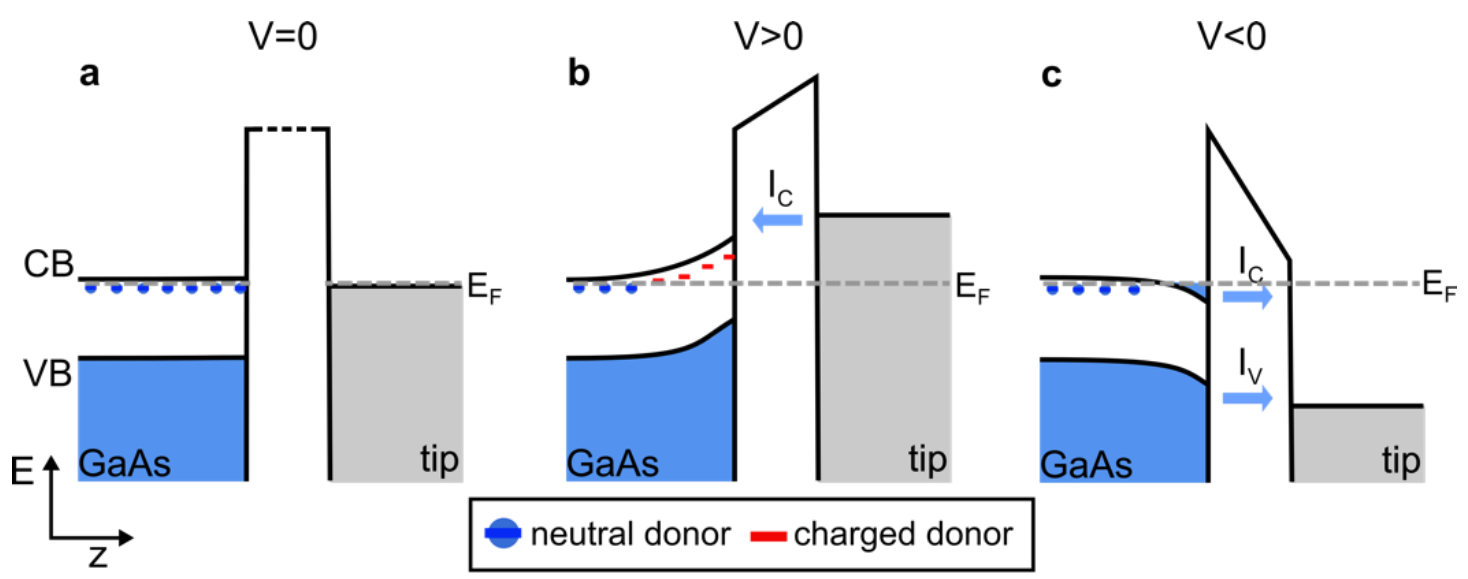

Fig. 1.5 Tip-induced band bending (TIBB) sketched in a tunneling band scheme for positive and negative bias voltage. (a) Band schemes of the STM tip and GaAs sample at zero voltage and macroscopically separated. (b) At positive voltages and in tunnel contact, surface-near dopants charge, resulting in a depletion layer at the GaAs surface. (c) At negative voltages the conduction band is pulled down below the Fermi energy, resulting in a confined electron gas at the surface.

At positive voltages (Fig. 1.5b), the potential, derived from the calculations in Fig. 1.4, has to be added. The bands and the donor levels beneath the CB are bent upwards, building up a localized depletion layer. Donors, which are pulled above the Fermi level, ionize, providing the charge for the screening of the tip potential. Regarding the tunneling process, empty states inside the $\mathrm{CB}$ are not directly addressable for voltages $\mathrm{V}>0$. Indeed one has to apply a certain voltage to overcome the TIBB and obtain the tunneling into the $\mathrm{CB} I_{\mathrm{C}}$. At lower positive voltages electrons have to additionally tunnel through the tip-induced space charge region. At negative voltages (Fig. 1.5c), $\mathrm{CB}$ and VB are bent downwards. Instead of depletion, an inversion layer at the surface is found. Already at small negative voltages the CB is pulled below the Fermi level, building a localized volume of filled states, in literature named as tip-induced quantum $\operatorname{dot}^{41}$. In this case the density of the accumulated electrons at the surface determines the screening length of the tip potential. Additional to the usual tunneling out of the $\mathrm{VB} I_{\mathrm{V}}$, the inversion layer allows the extra tunneling of electrons from the $\mathrm{CB} I_{\mathrm{C}}$. Please note that for p-type GaAs the voltage dependency reverses. 


\subsection{Surface Photovoltage and Scanning Tunneling Microscopy}

The Surface Photovoltage (SPV) describes the change in a surface potential due to the presence of photo-generated charge. Firstly reported in 1956 by Brattain et al. $^{42}$, it is widely used to characterize the electronic properties of photoactive materials in a non-contact method ${ }^{43}$. From the early days on, it found its application in extracting parameters like the minority carrier diffusion length, giving access to the defect density in the material ${ }^{4,44}$. This subchapter focuses on the SPV measured by local probe techniques.

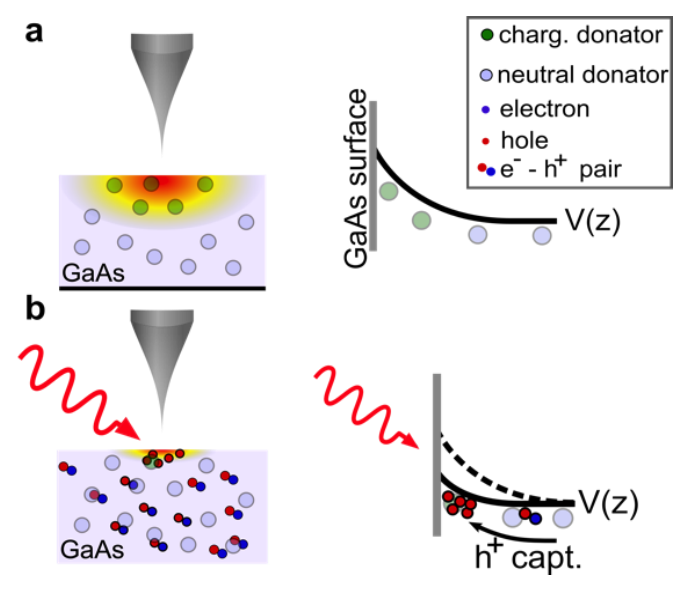

Fig. 1.6 Surface Photovoltage (SPV) in Scanning Tunneling Microscopy at the GaAs surface. (a) Under dark conditions the tip induces a localized potential at the surface. Surface near dopants are charged, screening the tip potential. (b) Optical excitation generates electron-hole pairs, which are separated by the tip-induced field. At positive bias voltages, holes accumulate beneath the tip, replacing the function of ionized dopants. The higher density of holes results in a more efficient screening of the tip potential. The change in the potential (dashed to solid line) is defined as the SPV.

Fig. 1.6 summarizes the main effects of the SPV generation in STM experiments at the GaAs(110) surface. A special hallmark of this surface is the lack of surface states, positioned inside the band gap. Consequently, the tip-induced potential (plotted color coded in Fig. 1.6a), is very sensitive to the total potential drop across the vacuum barrier ${ }^{40,45}$. It is important to note, that in the absence of the STM tip, the GaAs(110) surface is field-free. Therefore, without an approached tip closely to this surface no SPV effects are expected.

Under dark conditions and at positive bias voltages, the tip potential induces a depletion layer inside the GaAs surface. Surface-near dopants are ionized, providing the charge for the local SCR. Inter-band-gap-excitation at this surface results in the generation of electron-hole pairs. Analogue to a pn-junction, the photo-generated charges are separated inside the SCR. Holes accumulate at the surface beneath the STM tip whereas electrons drift back into the bulk-material (Fig. 1.6b). Being able to arrange freely and also having a higher charge concentration in comparison to the locally fixed dopants, the holes screen the tip-induced potential more efficiently. The holes at the surface take over the screening of the tip potential and the formerly ionized dopants discharge, leading to a reduction of the surface potential. This reduction in comparison to the potential under dark conditions is denoted as SPV. 
Another change of the surface potential, caused by optical generation of charge, is the Dember effect. In contrast to the SPV, the generated surface potential is a result of different diffusion characteristics of electrons/holes and is not induced by a surface field. Causing only minor potential changes ${ }^{5}$ in the order of only a few $\mathrm{mV}$, this effect is neglected within the scope of this thesis.

Two mechanisms define the absolute value of the SPV in Fig. 1.6b. On the one hand, it is determined by the tip-induced SCR, dependent on the applied bias voltage and contact-potential (including tip geometry) between STM tip and sample. At the GaAs surface this number is connected to the TIBB, which limits the maximum in downwards band bending during photoexcitation, before, so-called, flat band conditions for VB and CB are reached.

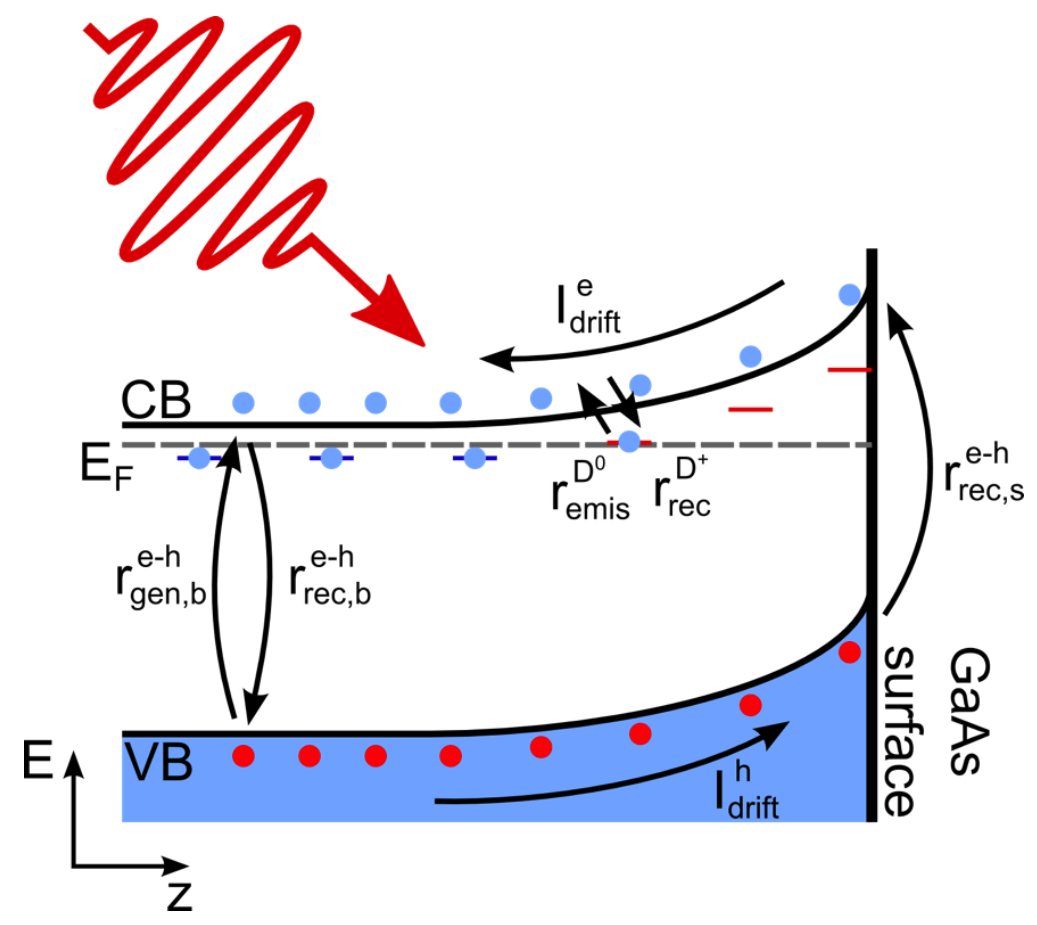

Fig. 1.7 Microscopic processes for the SPV. Optical excitation at the GaAs surface in the presence of an electric field results in complex dynamic processes. Besides electron-hole generation (recombination) in the bulk defined by the rates $r_{g e n, b}^{e-h}\left(r_{\text {rec,b }}^{e-h}\right)$, it includes hole (electron) transport via $I_{d r i f t}^{h}\left(I_{d r i f t}^{e}\right)$ to (from) the surface, recombination at the surface $r_{r e c, s}^{e-h}$ and local donor charging dynamics $\left(r_{e m i s}^{D^{0}}\right.$ and $\left.r_{r e c}^{D^{+}}\right)$.

One the other hand, the SPV is linked to the excitation density and intrinsic surface and bulk properties of the semiconductor. Optical excitation at a surface in the presence of a SCR may result in complex carrier dynamics (Fig. 1.7). Far from the surface, a steady state between electron-hole pair generation and recombination is expected, determined by the rates $r_{g e n, b}^{e-h}$ and $r_{\text {rec,b. }}^{e-h}$. The surface field induces charge transport of holes (electrons) to (from) the surface via the values $I_{d r i f t}^{h}$ $\left(I_{d r i f t}^{e}\right)$, determined, e.g., by the carrier diffusion length. Directly at the surface the concentration of holes is limited by the surface-site recombination $r_{r e c, S}^{e-h}$ mediated by surface or defect states. On top of that, charging dynamics of localized donors $\left(r_{e m i s}^{D^{0}}\right.$ and $\left.r_{\text {rec }}^{D^{+}}\right)$in the SCR play a crucial role. Under continuous excitation the quasi-equilibrium of the SPV at the surface is given by a steady 
state of all these microscopic processes. For defect-free GaAs, having long minority carrier diffusion lengths and no surface or defect states, it is known that already little charge generation rates are sufficient in order to discharge all ionized dopants inside the $\mathrm{SCR}^{11,46}$, leading to maximum downward band bending and thus to flat band conditions. More details on the concepts of SPV and resulting experimental methods can be found in, e.g., these two reviews ${ }^{4,47}$. The concepts of SPV will play a crucial role in the following main chapters. 


\section{Implementation of time resolution in STM techniques}

This chapter gives a detailed description on the implementation of time resolution in STM. After a short introduction to previous work, three prominent and already established realizations are presented. The pulsed optical excitation is discussed in more detail. At last, the setup, which was developed within the scope of the $\mathrm{PhD}$ period, is described and discussed.

\subsection{State of the art}

The prospect to access dynamic processes on the nanoscopic scale tempted several researchers to combine Scanning Probe Methods with pulsed excitation. Already in the early nineties, Nunez et al. proposed the use of optically triggered switches on the sample surface in order to control the tunnel current on the picosecond time scale ${ }^{48}$. A similar idea was made by Weiss et al. using photoconductive switches to generate fast voltage transients ${ }^{21,49}$. Hamers et al. followed a more conservative idea and implemented pulsed optical excitation directly at the STM tip to investigate dynamics of the local Surface Photovoltage (SPV) at surfaces with temporal resolution in the nanosecond scale ${ }^{50}$.

After these early attempts, progress was a long time coming. Worth mentioning is the effort of Grafström et al. ${ }^{51}$, trying to adapt and evolve the method of Hamers et al. However, in their work ${ }^{22}$, it was never possible to resolve processes via pump-probe excitation in STM due to optical-induced dominating thermal influences at the tip.

A renaissance of this topic started in 2004 and is based on the results of Shigekawa et al. With improvements in modern laser technology and the application of an elegant pump-probe excitation, they were able to resolve SPV dynamics at different semiconductor surfaces ${ }^{24,52-54}$.

This subchapter gives a brief overview of established setups and techniques allowing to access the evolution of dynamic processes on the nanoscopic scale. It starts with the method of pulsed electrical excitation, used in the research group of Andreas Heinrich (IBM research center, Almaden), followed by a recent technique, which uses Terahertz radiation. The end of the chapter focuses on the use of pulsed optical excitation and STM.

\section{Electrical excitation}

Firstly presented by Loth et al., the use of voltage pulses at the tunnel junction opened up a complete new method to investigate dynamic processes on the atomic scale ${ }^{55,56}$. This technique is 
based on the additional feeding of nanosecond voltage pulses to the dc bias voltage (Fig. 2.1). A pump pulse excites and drives the system out of equilibrium. A lower probe pulse senses the relaxation without further excitation. By averaging over the tunnel current for varying delay times between pump and probe pulses enables the access to the system decay, triggered via the pump pulse.

Fig. 2.1 Pump-probe scheme of voltage pulses at an STM tunnel junction. In this technique nanosecond long voltage pulses are applied in order to sense spin dynamics of single magnetic adatoms. Taken from ${ }^{55}$.

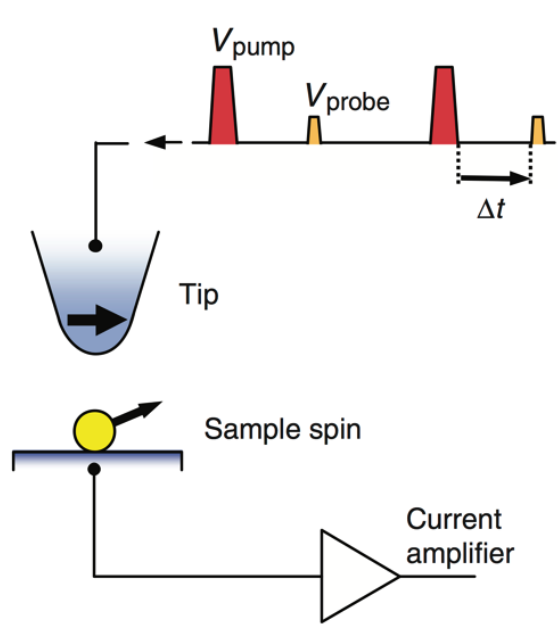

Loth et al. used this technique to study the relaxation process of excited spin states of a $\mathrm{Fe}-\mathrm{Cu}$ dimer on the $\mathrm{Cu}_{2} \mathrm{~N}$ surface (Fig. 2.2a). Performing $d I / d V$ spectra at the dimer resolves inelastic spin excitations at $+/-18 \mathrm{mV}$ (Fig. 2.2b). On a Cu adatom the $d I / d V$ curve is featureless, proofing the spin origin in the spectra.

Fig. 2.2c shows the spin relaxation of the $\mathrm{Fe}-\mathrm{Cu}$ dimer temporally resolved. Plotted are the electrons per probe pulse $\Delta N$ against the delay time $t_{\mathrm{d}}$ between pump and probe pulse. For $t_{\mathrm{d}}<-$ $300 \mathrm{~ns}$ the spin orientation of the magnetic adatoms and the spin-polarized tip are aligned. During excitation $\left(-300 \mathrm{~ns}<t_{\mathrm{d}}<0\right)$ the current drives the spin of the $\mathrm{Fe}-\mathrm{Cu}$ dimer in a non-equilibrium configuration. As the tunnel current is spin-polarized, it decreases within this time interval. For $t_{\mathrm{d}}>0$ the relaxation of the spin of the $\mathrm{Fe}-\mathrm{Cu}$ dimer to its ground state is recorded. The tunnel current converges to its value before excitation.

An advantage of this technique is the simplicity adapting this method to established STM setups. Moreover, in comparison to, e.g., optical methods, the excitation area is very localized, given by the tunnel current of the STM tip. However, further developments to increase the time resolution are limited by the bandwidth of the circuitry of the STM. In a follow up publication, Grosse et al. were able to resolve the actual pulse shape of the voltage pulse at the tunnel junction ${ }^{57}$. By reshaping the pulse, it was able to suppress effects like "ringing" due to the limited bandwidth or impedance mismatch. Saunus et al. ${ }^{56}$ presented an STM setup optimized for high frequency transmission and capable to generate pulses down to $120 \mathrm{ps}$ in duration. 

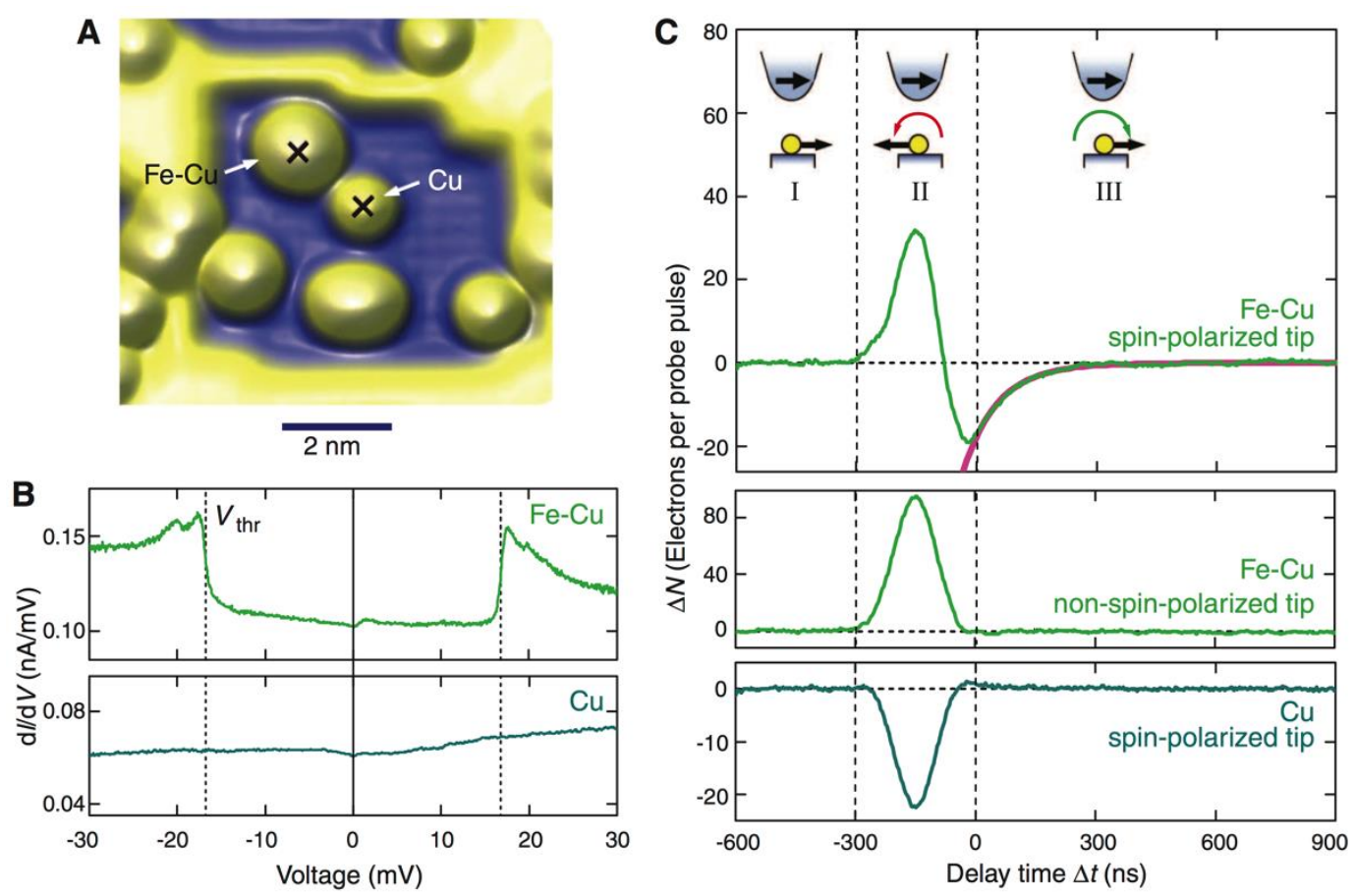

Fig. 2.2 Spin dynamics of $\mathrm{Fe}-\mathrm{Cu}$ dimers on the $\mathrm{Cu}_{2} \mathrm{~N}$ surface resolved on the nanosecond time scale. (a) Constant-current topography of single adatoms ( $\mathrm{Cu}$ and $\mathrm{Fe}-\mathrm{Cu}$ dimer) at the $\mathrm{Cu}_{2} \mathrm{~N}$ surface. (b) $d / / d V$ spectra on the $\mathrm{Fe}-\mathrm{Cu}$ dimer show inelastic excitations at $+/-18 \mathrm{mV}$. The same spectra at the $\mathrm{Cu}$ atom are featureless. (c) Additional current induced by pump-probe excitation (measured in electrons per probe pulse) plotted against the delay time between pump and probe with a spin-polarized tip on the Fe-Cu dimer. For reference, spectra with a non-polarized tip on the $\mathrm{Fe}-\mathrm{Cu}$ dimer or on the $\mathrm{Cu}$ ad-atom are shown (all measurements are performed at a perpendicular magnetic field of $7 \mathrm{~T}$ ). Taken from ${ }^{55}$.

\section{Terahertz excitation}

A hybrid between optical and electrical excitation in STM setups is using short Terahertz (THz) pulses. Fig. 2.3a shows an exemplary $\mathrm{THz}$ pulse, measured via electro-optical sampling. In the experiment the STM tip serves as a nano-antenna. The total voltage between tip and sample is composed of the dc bias voltage and the voltage transient induced by the THz pulse (Fig. 2.3b, c). The non-linear current dependency of the tunnel current on the bias voltage (Fig. 2.3e) rectifies the additional THz-induced and originally symmetric voltage pulse. $I(V)$ curves on highly orientated pyrolytic graphite (HOPG) indicate the influence of the THz radiation (Fig. 2.3f). As an exemplary sample system Cocker et al. ${ }^{58}$ used the carrier generation and relaxation at InAs nanodots. By exciting the surface with high intensity optical pump pulses, they were able to map the hot carrier relaxation in the picosecond time regime. 

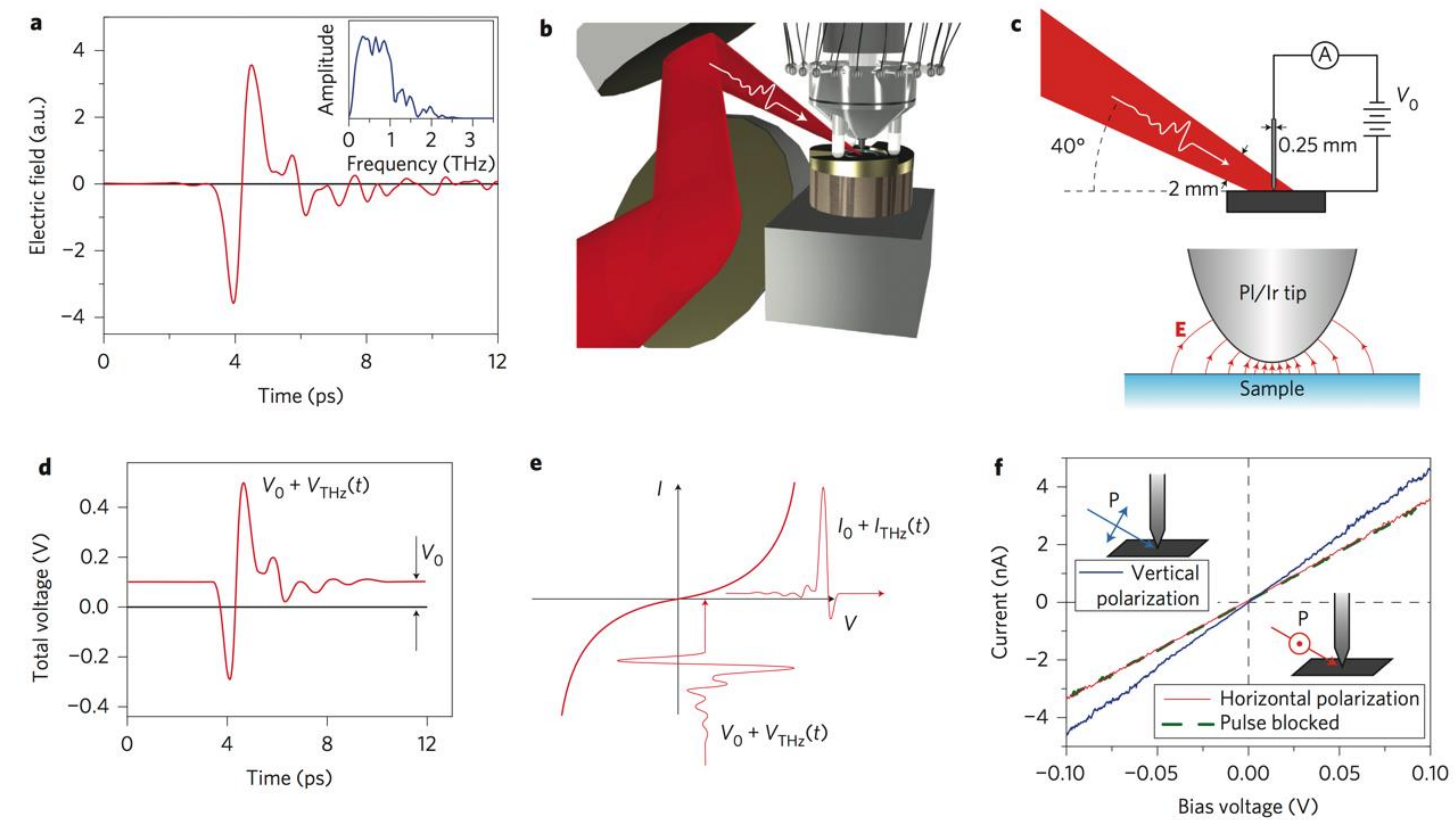

Fig. 2.3 Terahertz (THz) radiation in STM experiments. (a) THz pulse measured by electro-optical sampling. Inset: Corresponding frequency spectrum. (b, c) THz pulses are focused into the tunnel junction. The STM tip acts as a nano-antenna. (d) Total voltage at the tunnel junction: DC bias voltage plus THz induced voltage. (e) The nonlinear dependency of the tunnel current on the bias voltage rectifies the symmetric $\mathrm{THz}$ pulse. (f) $/(V)$ curve, taken at an HOPG surface with and without $\mathrm{THz}$ radiation, illustrates the plus in voltage due to the $\mathrm{THz}$ pulse. Additionally, the $\mathrm{THz}$ pulse was focused horizontally polarized into the junction, having the same effect like no excitation. Taken from ${ }^{58}$.

THz excitation in STM has great prospects in order to overcome the drawback of the bandwidthlimited circuitry in STM setups. However, this technique is still in its infancy. A first promising application was done by Cocker et al. resolving the periodic motion of an isolated adsorbed molecule ${ }^{59}$.

\section{Optical excitation}

In the year 1990, Hamers et al. made first implementations of pulsed optical excitation in Scanning Probe Methods ${ }^{23}$. The intention was resolving the dynamics of the SPV at the Si(111)-7x7 surface, which was already investigated under continuous excitation ${ }^{60}$.

Instead of the formerly used photo-potentiometry, by comparing the increase in current at a distinct bias voltage, Hamers et al. performed Scanning Capacitance Microscopy ${ }^{61}$ with the tip more than $100 \mathrm{~nm}$ separated from the sample surface (Fig. 2.4a). The change in the surface potential due to build-up of a photovoltage is measured as a displacement current. By chopping the laser illumination in "on" and "off" cycles, the resulting modulation in this current is extracted via lockin amplification. Fig. 2.4b shows experimental results of this method indicating a clear dependency on the delay time between pump and probe pulses. 


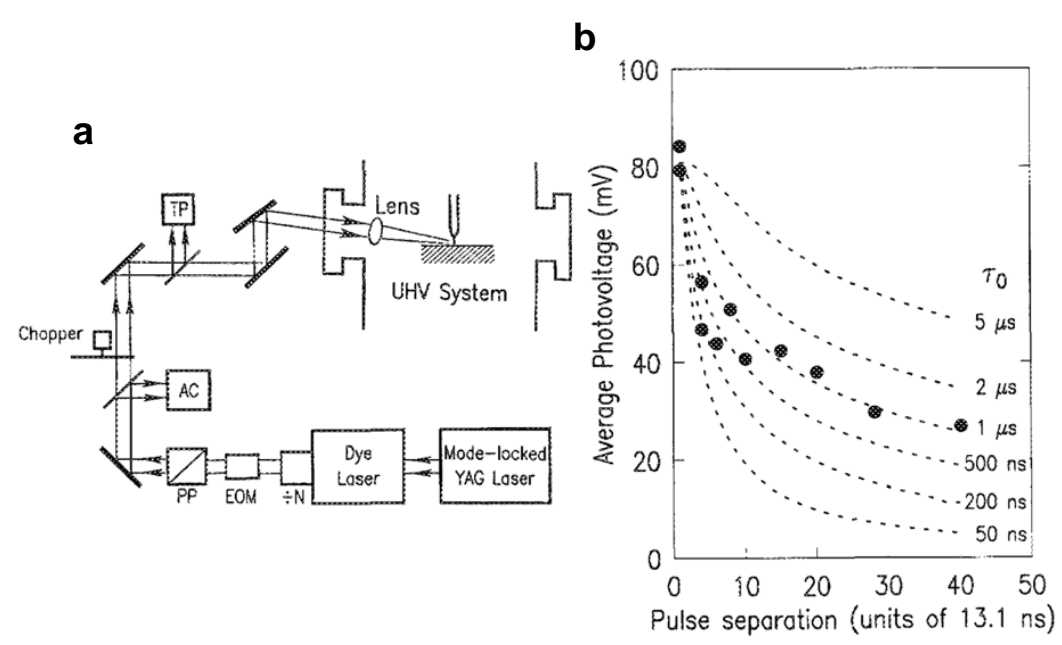

Fig. 2.4 Optical setup and results of the relaxation of the photo voltage, time resolved at the $\mathrm{Si}(111)-7 \times 7$ surface. (a) A mode locked laser with a pulse width below 1 ps and a repetition cycle of $13 \mathrm{~ns}$ is focused onto STM tip and sample surface. Pulse picking allows for generating double pulse pairs separated by multiples of the repetition cycle. (b) Experimental results of the SPV temporally resolved. Taken from ${ }^{23}$.

Weiss et al. invested substantial effort to implement direct optical excitation during STM operation. The main challenge here was the handling of thermal issues at the STM. They performed frequency dependent measurements of the tip expansion in tunnel contact at a polished gold surface (Fig. 2.5). In a frequency range from 1 to $10^{5} \mathrm{~Hz}$, the tip expands of at least $100 \mathrm{pm}$ per $\mathrm{mW}$ towards the sample surface, which induces a change in the tunnel current of a factor of ten for a typical tunnel barrier of $4 \mathrm{eV}$. For higher frequencies the expansion per $\mathrm{mW}$ decreases. In the year 2000 Gerstner et al. published a report that indicates mainly thermal tip effects in the tunnel current even in the femtosecond time regime ${ }^{62}$. Consequently, Weiss et al. stated in 2002 that they have never been able to resolve dynamic processes due to dominating thermal effects ${ }^{22}$.

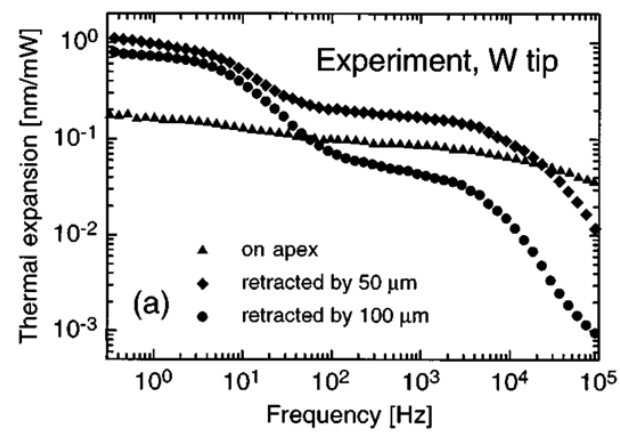

Fig. 2.5 Thermal expansion of the STM tip due to optical excitation. Frequency resolved tip expansion for optical excitation at the tunnel junction. Taken from ${ }^{51}$.

In the year 2004, the first successful implementation of pulsed optical excitation in STM during operation was made by the research group of Hidemi Shigekawa at the University of Tsukuba ${ }^{24}$. In order to solve the problems induced by thermal effects, they invented a very elegant method, called shaken-pulse-pair excitation (SPPX), which keeps the thermal load at the tip constant. The experimental method in this thesis is based on this technique. Therefore preliminary work will discussed in detail. 
Fig. 2.6 First optical setup of Shigekawa et al. in order to generate the shaken-pulsepair-excitation. With the help of a beam splitter and a delay line, pulse pairs are generated by a mode-locked Ti:Sapphire laser. One of the mirrors of the delay line can be periodically moved in position, inducing a modulation of the delay time of the second pulse. Taken from ${ }^{24}$.

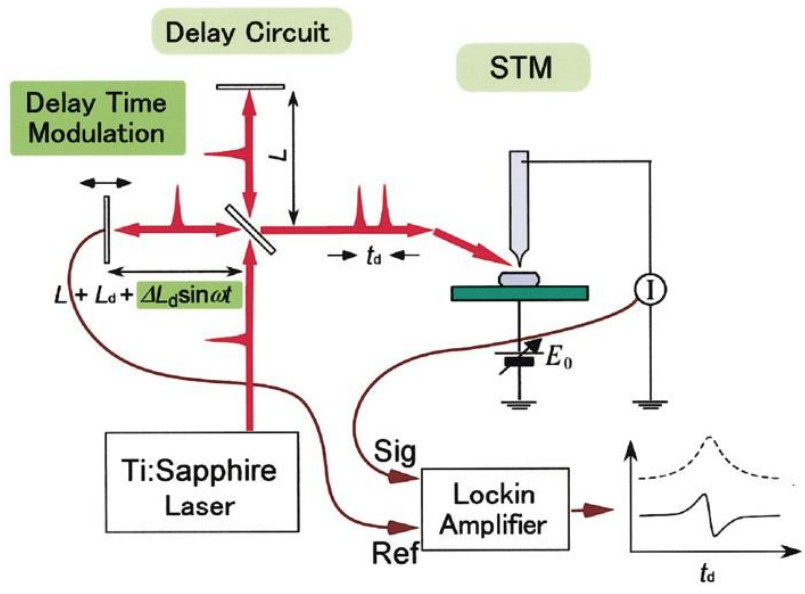

A sketch of their first optical setup is pictured in Fig. 2.6. This setup is based on a mode-locked Ti:Sapphire oscillator generating femtosecond pulses. With a beam splitter and a delay line, these single pulses can be combined to a pulse pair with a variable delay time in between. The "trick" of the SPPX is the periodic modulation of this delay time, realized in this setup via a periodic movement of one of the mirrors in the delay line. Fig. 2.7 sketches how to extract temporal information from this double pulse experiment with modulated delay times. If a decaying process is triggered on each pulse (red shades), a certain amount of the system response will overlap. By modulating the delay time periodically, this overlap also varies. The absolute variation of this overlap during modulation is dependent on the decaying behavior of the response and can be extracted via lock-in amplification. In order to access $d I$ induced by the pulsed excitation, numerical integration is needed for this method.

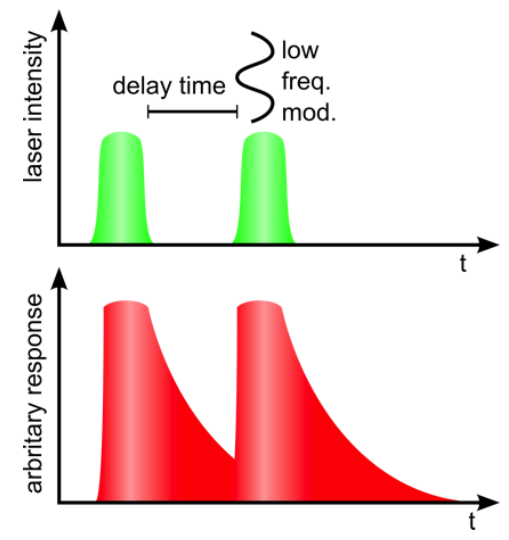

Fig. 2.7 How to extract temporal information in pump-probe experiments with a periodically modulated delay time. Upper part: Optical pulse pair. By a periodic movement of the mirror in the delay line, the delay time between both pulses is modulated. Lower part: Arbitrary non-linear response to the pulsed excitation. Depending on the delay time the response to the pump and probe pulses overlap. According to the modulation of the delay time this overlap varies.

Shigekawa et al. have improved this method considerably in the last twelve years. The mechanical movement of the mirror in the delay line induces a high amount of noise in the signal, demanding long averaging times at each delay point. They decided to use a sophisticated pulse picking technique, sketched schematically in Fig. 2.8 ${ }^{25}$. The basis of this setup are two synchronized Ti:Sapphire oscillators, capable of providing femtosecond laser pulses and having a repetition rate of $90 \mathrm{MHz}$. Each laser oscillator provides pump or probe pulses, respectively. By pulse picking with fast Pockels cells, delay times between pump and probe can be generated, which correspond 
to multiples of the repetition cycle. For delay times in between the repetition cycle both oscillators can be detuned to each other, covering the time range between $1 \mathrm{ps}$ and $11 \mathrm{~ns}$.

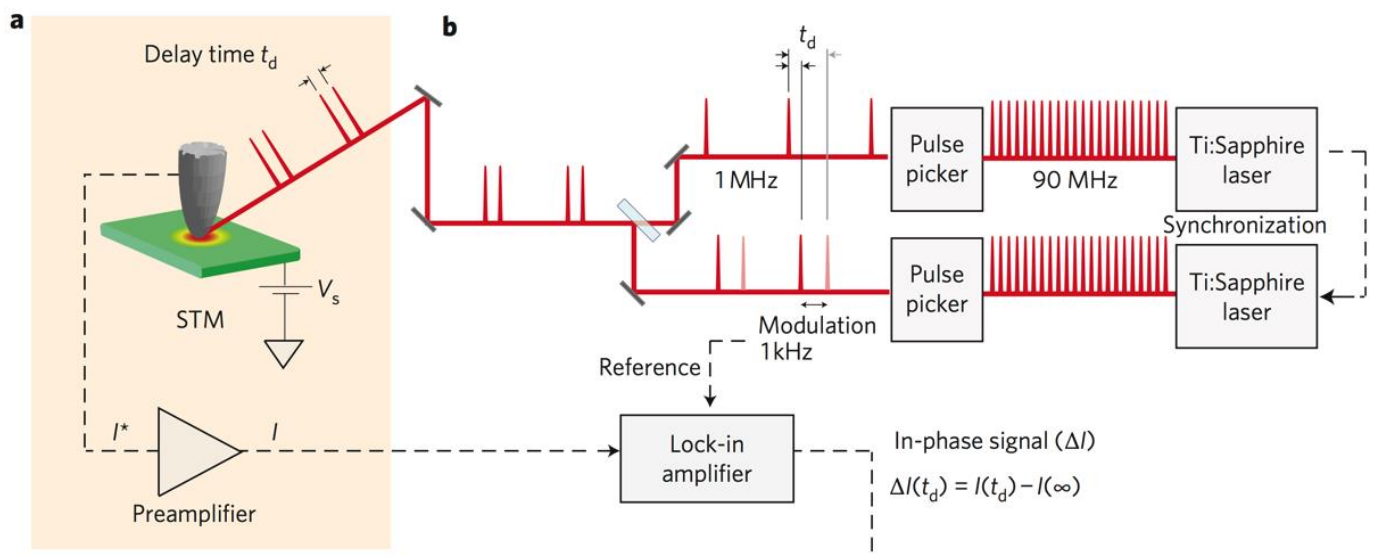

Fig. 2.8 Schematic of the SPPX generation with the help of two femtosecond laser oscillators. By pulse picking with fast Pockels cells, the pump probe pattern is generated. The delay time of the pulse pair switches between a short, variable and a long, fixed and well-separated value. Taken from ${ }^{52}$.

The low-frequency modulation of the delay time, necessary for the SPPX, is realized by switching the delay time between short values and long values. As a result and in contrast to Fig. 2.6 the modulation of the delay time is not sinusoidal anymore. Instead, the delay time jumps periodically between short and long values. A more detailed description of the pulse pattern can be found in subchapter 2.2.

This setup enabled the detection of charge dynamics at the $\operatorname{GaAs}(110)^{25,63,64}$ and other surfaces ${ }^{54}$ on the picosecond and nanosecond time scales. These results are relevant for the experimental outcome presented in this thesis. A detailed review is given in chapter 4.1- 4.3.

Only recently, Dolocan et al. stated in their report ${ }^{65}$ that even the SPPX method does not solve the problem of thermal tip shrinking/expanding effects. The authors propose to use a two-color (frequency-doubled) SPPX, where the probe pulse is frequency-doubled in comparison to the pump pulse. Phase matching of both pulses hinders the build-up of interferometric thermal modulations during femtosecond pulses. In conclusion, they question the results of Shigekawa et al. ${ }^{52}$ highly.

\subsection{A versatile implementation of pulsed optical excitation in Scanning Tunneling Microscopy}

A major part of this $\mathrm{PhD}$ thesis comprises the development of an optical setup, providing nanosecond pulsed excitation and probing, and its successful implementation during STM operation. Instead of highly sophisticated femtosecond oscillators, we decided to generate nanosecond pulses purely electronically and then shape the output of a continuous wave laser diode 
into pulses temporally by a wideband electro-optical modulator. This implementation bears a great versatility with regards to the tuning of excitation parameters to the experimental needs.

This subchapter is published in identical words in the scientific journal "Review of scientific instruments". To simplify the readability of this thesis, a brief summary will be given in the following section. Additionally, at the end of this chapter, a general discussion can be found.

Parts of the experimental results as well as of the implementation were done in collaboration with former bachelor and master students. In detail, the implementation of the movable stages in the setup was a part of the bachelor thesis of Ole Bunjes. The measurements of the thermal expansion of the STM tip were done in collaboration with Terence Thias. A detailed analysis of the noise characteristics of the laser diode was done within the master thesis of Judith von der Haar.

\section{Brief summary}

A key-element to access dynamic processes with time constants far above the bandwidth of the STM is the SPPX. This methods describes a periodic modulation of the delay time between pump and probe pulse. In our implementation the SPPX pattern is purely generated electronically. We use a high frequency arbitrary waveform generator in combination with a high bandwidth electrooptical modulator. Our implementation bears a great versatility: Excitation parameters such as pulse width, delay time or pulse intensity can be adjusted instantly on the fly to the experimental needs.

For the optical setup, a low-noise laser source is essential. Already little fluctuations of the intensity can lead to thermal instabilities at the tunnel junction making STM operation impossible. In order to position the focus of the laser beam into the tunnel junction we have installed submicrometer precise translation stages.

By raster-scanning the focus point across the sample and STM tip geometry, we investigate the absolute thermal expansion of the STM tip which can be estimated to values between 10 to $100 \mathrm{~nm}$ (depending on the focus position and laser power). After thermalization, stable STM operation is possible.

For the first time it was possible to quantify the contribution of thermal effects during pulsed excitation. For the n-doped GaAs(110) surface only at positive sample voltages a SPV is expected. At negative voltages pure thermal effects can be studied.

Raster scanning the focal spot of the laser and recording the optically induced current in pulsed mode, generates current maps, which reveal a significant difference in the lateral signal distribution between both bias voltages. Whereas we observe a very localized signal situated at the tip apex for positive voltages, the spatial extend of the signal at negative voltages (thermal contribution) follows the elongated symmetry of the STM tip. The maximum thermal effects and the maximum 
SPV-induced effects are locally well separated. A quantitative analysis directly at the tip apex shows that the ratio of the signal between positive (time-resolved SPV) and negative bias voltages (thermal origin) is $20: 1$.

As a proof of principle, single time spectra are taken at the GaAs(110) surface for positive sample voltages showing a significant inhomogeneity of the SPV dynamics on the atomic scale.

\section{Original publication}

We present a combination of pulsed optical excitation and Scanning Tunneling Microscopy. A great versatility of this setup is given by the method of optical pulse generation. A gigahertz electro-optical modulator, which is driven by a high frequency arbitrary wave generator, processes a continuous-wave laser beam of a low-noise laser diode into the desired wave shape. For pump-probe excitation we generate optical pulse series in an all-electronic way. Thereby we can easily adapt parameters like pulse amplitude, width or repetition cycle to the demands of the experiment. This setup is used to study different dynamic processes at the GaAs(110) surface. We separate thermally from electrically induced effects, allowing us, for the first time, to quantify the thermal contribution of the optical excitation in STM experiments. Time-resolved decay spectra of the photo-generated electron-hole pairs demonstrate the nanoscale spatial resolution.

P. Kloth, T. Thias, O. Bunjes, J. von der Haar and M. Wenderoth, Rev. Sci. Instrum. 87, 123702 (2016), DOI: $10.1063 / 1.4971189$.

\section{Introduction}

The combination of optical excitation and scanning probe methods has been standing in the focus of research since the invention of the Scanning Tunneling Microscope (STM) ${ }^{4,50,60,66-68}$. The prospect to access optical properties at surfaces with a resolution way beyond the diffraction limit inspired various interesting implementations ${ }^{24,48,50,69-72}$. With the advancements in laser technology and the groundbreaking results of Shigekawa et al., modern studies succeeded to implement pulsed laser excitation in STM operation ${ }^{16,24,25,71}$. It is worth mentioning that thermal effects at the STM tip have been a major problem since the beginning and are still under discussion ${ }^{51,65,73}$.

This review presents a versatile laser setup for pulsed optical excitation in STM, which is organized as follows. First, we explain the experimental method to access temporal resolution in STM. Apart from the technical description of pulse generation, we highlight the advantages of the realization with respect to its flexibility. Next, the optical setup is sketched. With the STM tip in tunneling contact, variations in the excitation power, leading to thermal fluctuations, have to be avoided. To demonstrate the operational performance of our implementation, we have probed different processes at the GaAs(110) surface. We quantify the contribution of thermally induced effects and 
study the carrier dynamics at this surface. Single pump-probe spectra of the photo-excited GaAs surface are shown, revealing a significant inhomogeneity on the atomic scale.

\section{Shaken-Pulse-Pair-Excitation}

In order to acquire temporal information of dynamic and reversible processes with characteristic time constants below the bandwidth of the detector (in our case the tunnel current $I$ ), the so-called pump-probe excitation is applied. The principle idea of this mechanism is sketched in Fig. 2.9a, consisting in our setup of two identical pulses (Fig. 2.9a, green dotted rectangles) separated by a variable delay time $t_{\mathrm{d}}$. The response in the tunnel current $I$ (red shades) to both pulses overlaps, depending on $t_{\mathrm{d}}$. Due to the low bandwidth of the current amplifier, not the real-time response $I$ but instead the averaged value $\langle I\rangle$ (Fig. 2.9a, blue curves) is measured. $\langle I\rangle$ is directly connected to the overlapping of the real-time response. Consequently, measuring $\langle I\rangle$ for various $t_{\mathrm{d}}$ (Fig. 2.9b) gives access to the dynamics of the system.

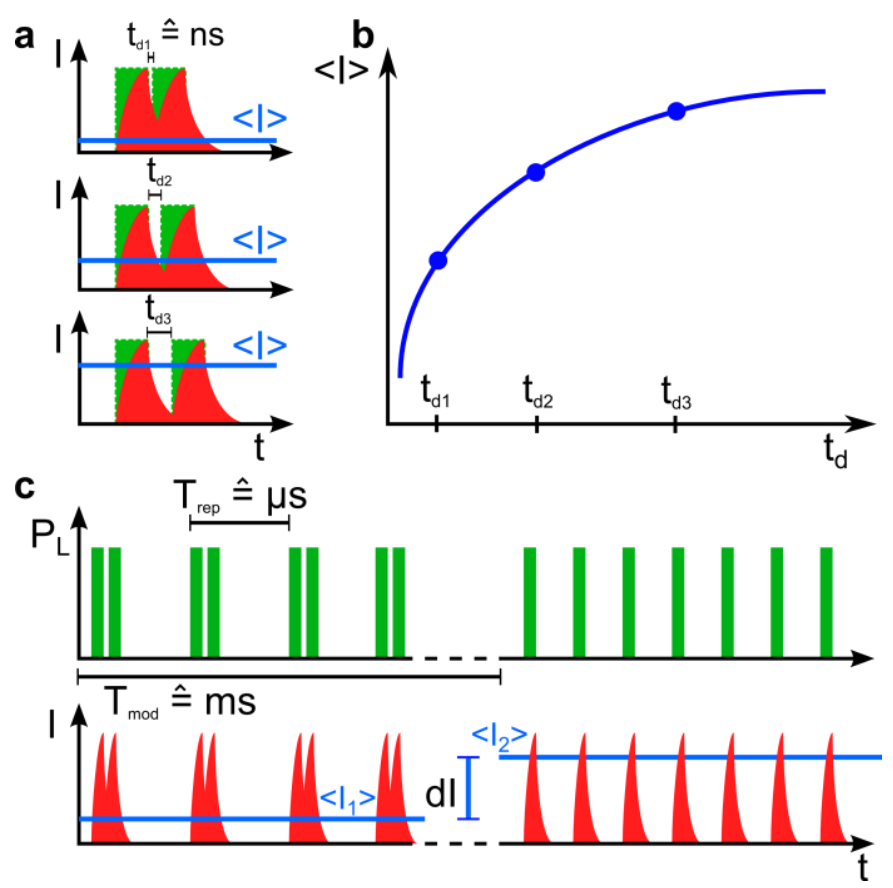

Fig. 2.9 Pump probe method and shaken-pulse-pair-excitation.

(a) Pump-probe excitation (green dots) superimposed with an arbitrary real-time response I (red) of a triggered dynamic process. Additionally, the corresponding averaged value (blue line) is indicated. (b) Averaged value in (a) plotted against the delay time $t_{d}$. (c) A periodic modulation of the delay time $t_{\mathrm{d}}$ defines the Shaken-pulse-pair excitation.

Due to small signal to noise ratios and possible thermal effects, $\langle I\rangle$ cannot be measured in that simple way, e.g. at a fixed tip-sample-distance with deactivated feedback loop, while varying $t_{\mathrm{d}}$. Instead, an appropriate lock-in amplification scheme is necessary. In order to add a repetitive modulation to the pump-probe excitation, the group of H. Shigekawa at the University of Tsukuba applied a very elegant implementation, called shaken-pulse-pair excitation (SPPX) ${ }^{24,25,53}$. The main idea is a low-frequency periodic variation of the delay time $t_{\mathrm{d}}$ between pump and probe pulse, sketched in Fig. 2.9c. Determined by the modulation cycle $T_{\text {mod }}$, the double pulse pair switches between two patterns. One is given by two separated pulses with a variable delay time $t_{\mathrm{d}}$ in between. In the second pattern, serving as the reference, the two pulses are separated by their maximum time, i.e. half of the repetition cycle $T_{\text {rep }}$ of the pump-probe pairs. This method has the 
advantage that the time averaged thermal load at the tunnel junction, induced by the laser (averaged $\mathrm{t}>T_{\text {rep}}$ ), remains constant (please note that in contrast to the thermal load, the thermal response of the system is not constant.). Averaging the real-time response according to the bandwidth of the experiment, given by the I/U converter, results in a periodical modulation of the tunnel current between $\left\langle I_{1}\right\rangle$ and $\left\langle I_{2}\right\rangle$ (Fig. 2.9c, blue curves) where the difference $d I=\left\langle I_{1}\right\rangle-\left\langle I_{2}\right\rangle$ is determined by the overlap of the real-time response in case of the shortly-separated pulses. Analogue to Fig. 2.9a, by varying $t_{\mathrm{d}}$ and extracting the amplitude of the periodic signal $d I$ by lock-in detection, the decay of the real-time response can be measured.

\section{Pulse generation in an all-electronic way}

In order to generate the SPPX pattern, Shigekawa et al. use a pulse picking technique including two femto-second laser oscillators and several high voltage Pockels cells ${ }^{16}$. This setup includes drawbacks in regard to its flexibility by adapting the excitation parameters, like pulse width or repetition cycle, to the experimental needs. In order to be more flexible, we decided to generate the SPPX pattern purely electronically using a high frequency arbitrary wave generator (AWG) and process the laser beam of a continuous-wave ( $\mathrm{cw}$ ) laser diode in pulsed shape by a fiber-coupled electro-optical modulator (EOM). A benefit of this approach is that the EOM is operated at low voltages $(\mathrm{U}<5 \mathrm{~V})$ avoiding high frequency / high voltage parasitic signals in the lab.

Fig. 2.10 Schematic of the SPPX generation via pulse width modulation. (a) The amplitude of a low frequency signal $U_{\text {mod }}$ (red shade) modulates the duty cycle of a (b) square wave $U_{\text {rep }}$ (green shade). The duty cycle of $U_{\text {rep }}$ defines the delay time $t_{\mathrm{d}}$. The frequency of $U_{\text {rep }}$ gives the repetition cycle of the pump and probe pulse pair. (c) Short pulses (blue shade), triggered on each rising or falling edge of $U_{\text {rep }}$, give the SPPX pattern $U_{\text {SPPX. }}$. (d) For low bandwidth systems, the response to this excitation (grey pulses) is averaged resulting in (e) a sinusoidal shaped waveform (yellow curve), modulated with the frequency of $U_{\text {mod. }}$.

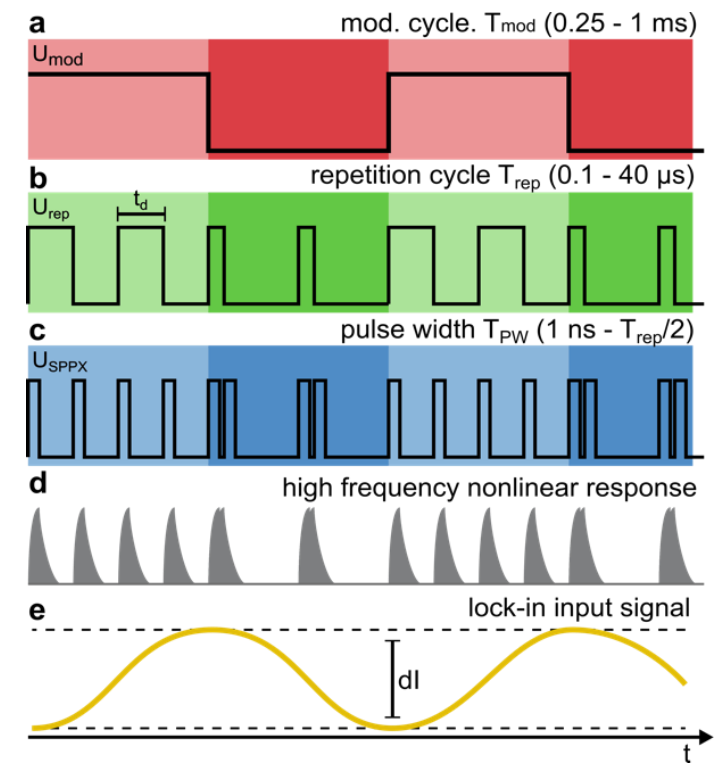

The corresponding electronic signal chain is schematically shown in Fig. 2.10. A low frequency square wave $U_{\text {mod }}\left(\mathrm{T}_{\bmod }=0.25-1 \mathrm{~ms}\right.$ cycle) (Fig. 2.10a) modulates the pulse width of $U_{\text {rep }}$. The frequency of $U_{\text {rep }}$ defines the repetition cycle $\left(\mathrm{T}_{\text {rep }}=0.1-40 \mu \mathrm{s}\right)($ Fig. 2.10b) of the pump-probe pulse pair. $U_{\text {rep }}$ has a modulated duty-cycle synchronized to the periodicity of $U_{\text {mod. }}$. By triggering pulses on each rising and falling edge of $U_{\text {rep }}$, the resulting pulse scheme $U_{\text {SPPX }}$ is generated, divided into two patterns (Fig. 2.10c), analogue to Fig. 2.9c. Taking an exemplary response $I$ (Fig. 2.10d, grey pulses) and considering the limited bandwidth of the tunnel current $(<6 \mathrm{kHz}), I$ 
transforms to a periodic modulation with the amplitude $d I$ (Fig. 2.10e, yellow line). The periodicity is given by $T_{\text {mod. }} d I$ is phase-sensitively measured via lock-in amplification, with $U_{\text {mod }}$ being the reference. By generating the whole SPPX pattern purely electronically, we are able to easily adjust parameters like the pulse width, the pulse repetition cycle or even the pulse height, giving the excitation intensity of the optical pulses, during STM operation.

\section{Optical setup}

Basis of our optical system (Fig. 2.11a) is a low noise continuous-wave (cw) laser diode (100 mW max. power, $\lambda=785 \mathrm{~nm}$ ) with a long-term RMS noise value below $0.02 \%$ of the output power. After fiber coupling, the laser beam is processed by an electro-optical modulator (EOM).

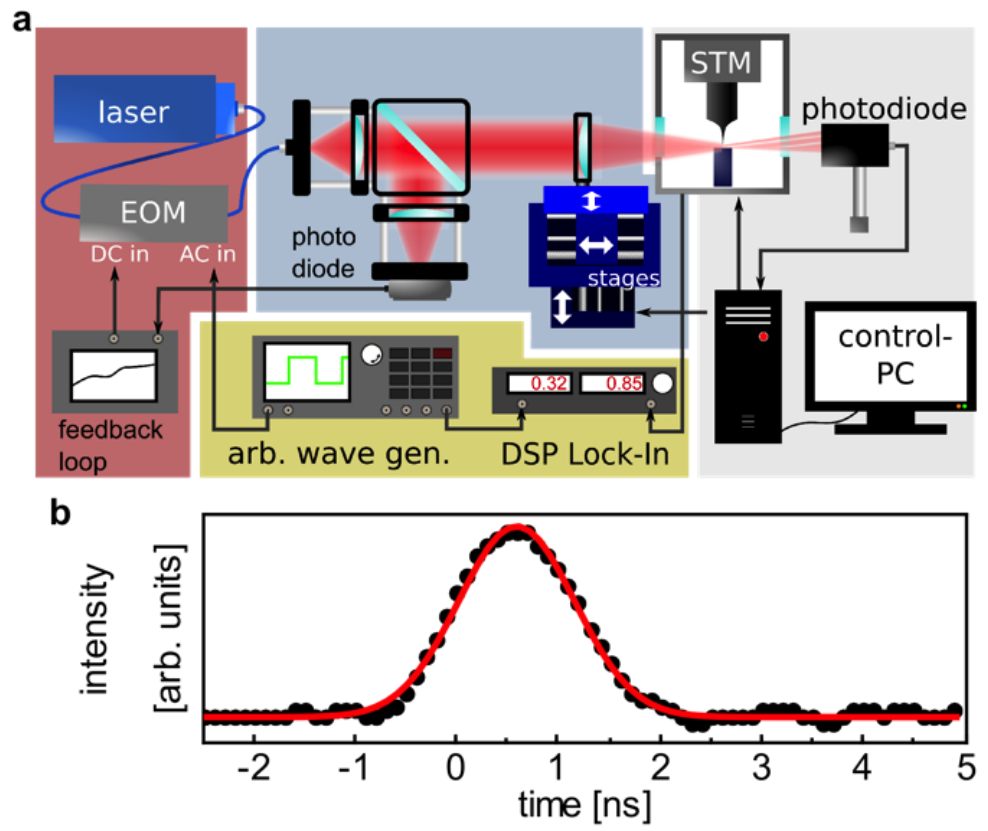

Fig. 2.11 Schematics of the optical setup. (a) A schematic overview of the optical setup. The implementation can be divided into three parts. The first part (red shades) shows the components for generating modulated laser light from a continuous-wave laser diode with the help of an electro-optical modulator (EOM). The blue shade includes the optical setup at the STM chamber in order to focus the laser beam into the tunnel junction. We use sub-micrometer controllable stages to fine position the focus point in $x$-, $y$ - and $z$-direction. Additionally we have installed a photo diode in order to monitor the optical excitation. The electronic implementation includes the pulse generation by the arbitrary wave generator and signal detection by lock-in amplification (yellow shades). (b) The shortest optical pulse generated by our optical setup. The FWHM is 1.6 ns defining the time resolution in our experiments.

Having a bandwidth of $20 \mathrm{GHz}$, the EOM in combination with the voltage signal given by the AWG transforms the laser beam into any desired shape, from continuous over low frequency periodic to nanosecond pulsed excitation with variable amplitude. The shortest optical pulses generated with our setup can be seen in Fig. 2.11b. Recorded with a fast photo diode (300 ps rise/fall time) it has a full width at half maximum (FHWM) of $1.6 \mathrm{~ns}$ defining the time resolution of our setup. As the bandwidth of the EOM is specified to $20 \mathrm{GHz}$, a faster electronic pulse generation 
should be able to increase the time resolution remarkably. For positioning the laser beam in the vacuum chamber into the tunnel junction we use a sub-micrometer precise moving $(x-, y$ - and $z$ direction) stage (Fig. 2.11a, blue shade).

This setup requires a compensation of the bias voltage drift $U_{\text {offset }}$ of the EOM. In order to monitor the optical output of the EOM we installed a beam splitter and a fast photo diode into the beam path before focusing into the tunnel junction. Analogue to ${ }^{74}$, we apply a feedback loop to set the optical power to a constant value via an additional dc bias input ( $\left.U_{\text {offset }}\right)$ counteracting the voltage drift of the EOM. Finally, the extinction ratio of the EOM is 1:200.

\section{Pulsed optical excitation at the GaAs(110) surface}

A key task in the pulsed optical excitation in tunneling microscopy is the separation of thermally ${ }^{73}$ (e.g. tip expansion/shrinking) and electronically induced effects (e.g. optical charge generation). All following measurements are conducted at a base temperature of $77 \mathrm{~K}$ at the n-doped GaAs(110) surface $\left(n_{\mathrm{D}}=3 \cdot 10^{18} / \mathrm{cm}^{3}\right)$ with chemically etched tungsten tips. In a first step, we monitor the absolute z-shift of the STM tip during raster-scanning the focus of the laser beam (continuous excitation) across the tunnel junction (Fig 2.12a). The tip geometry with the shaft at the bottom of the figure and the etched area on top of it can be identified. Depending on the focus position, the thermal expansion of the tip varies between $10 \mathrm{~nm}$ (at the apex) to $100 \mathrm{~nm}$ (at the shaft region). However, after thermalization of the STM tip, stable tunneling with atomic resolution is possible independent of the position of excitation.

It turned out, that for an exact positioning of the laser focus at the tunnel junction, a different method is more practical. Using a sinusoidal, low frequency modulation $(>1 \mathrm{kHz}$ and $<6 \mathrm{kHz})$ of the laser intensity, we induce a modulation $d I_{\text {lowfreq }}$ in the tunnel current $I$. By raster-scanning the focus point (focus diameter $<20 \mu \mathrm{m}$ ) across the tip, we picture the tip-sample geometry by recording $d I_{\text {lowfreq }}$, providing us the position of the apex of the STM tip (Fig 2.12b). As a reference, Fig 2.12c shows the image of an etched tungsten STM tip, recorded with a Scanning Electron Microscope (SEM). For this low-frequency modulated laser intensity, we mainly expect thermal effects as the reason for the change in the tunnel current. The major part of the induced signal is compensated by the feedback loop of the STM.

In order to investigate the thermal high frequency response of the tunnel junction, we applied pulsed optical excitation at the tunnel junction. The focus point of the laser beam is raster-scanned along the tunnel junction and the resulting $d I_{\mathrm{SPX}}$ is laterally recorded for both, positive (Fig 2.12e) and negative bias sample voltages (Fig 2.12f). The delay time between pump and probe pulse was adjusted to the minimum of $t_{\mathrm{d}}=1.6 \mathrm{~ns}$. At positive bias we observe $d I_{\mathrm{SPPX}}$ as a roundly shaped signature directly at the tip apex. In contrast, at negative voltages, the contour of the $d I_{\mathrm{SPPX}}$ signature follows the elongated symmetry of the STM tip. As a reference, Fig 2.12d shows a 
magnified view on the STM tip for low frequency modulated laser intensities, analogue to Fig 2.12b.

Fig 2.12 Raster scans of the focus point across the tip and sample. (a) Absolute $z$-shift of the STM tip due to thermal expansion when raster-scanning the focus of a continuous-wave laser along the tunnel junction (set point $-2 \mathrm{~V}$, $10 \mathrm{pA}$, averaged excitation power $1.2 \mathrm{~mW}$, focus diameter $20 \mu \mathrm{m}$ ). (b) Raster scanning the focus point across the tunnel junction maps the geometry of the tip and sample. The laser intensity is modulated into a low frequency, sinusoidal wave shape inducing a modulation in the tunnel current $d l_{\text {lowreq }}$ (plotted color coded against focus position). (c) A comparable Scanning Electron Microscopy picture of an etched tungsten tip for reference. (d) Zoom on the low frequency excited tunnel junction, analogue to (b). (e, f) Focus raster scans across the tunnel junction. SPPX is used with a minimum delay time between pump and probe pulse (set point $2 \mathrm{~V} /-2 \mathrm{~V}, 50 \mathrm{pA}$, exc. parameter: rep. cycle $5 \mu \mathrm{s}$, pulse width $80 \mathrm{~ns}$, averaged excitation power $70 \mu \mathrm{W}$, focus diameter $20 \mu \mathrm{m}$ ). The red circle marks the position of the tip apex. The red box marks the position with the maximum of thermal effect. (g) $d l_{\text {SPPX }}$ at negative
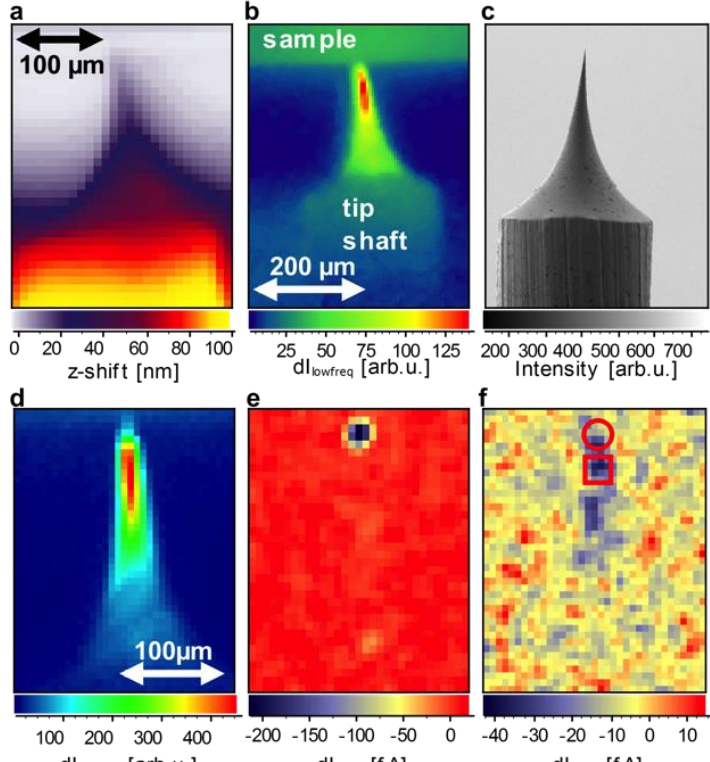

dl $_{\text {bwfreq }}$ [arb.u.]
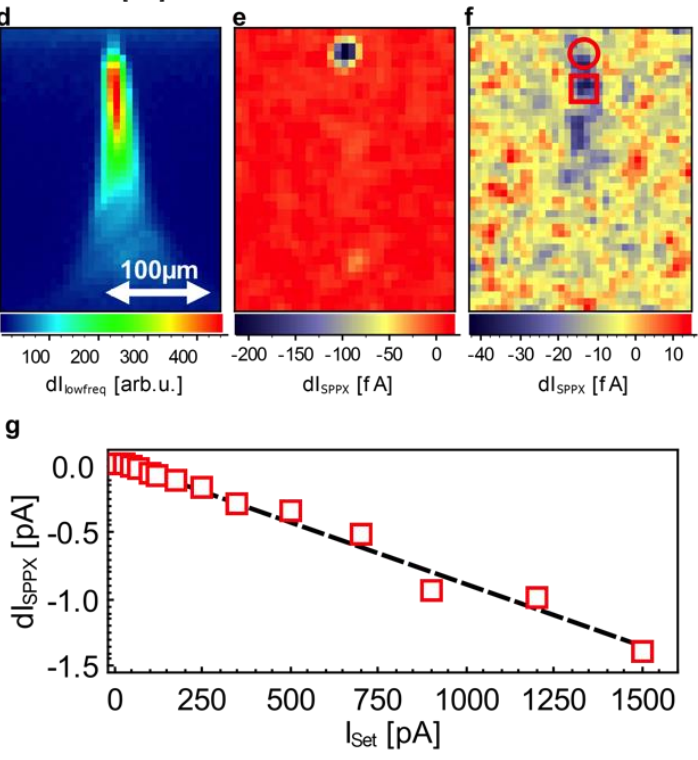
bias voltages evaluated inside the red box for increasing set point currents $I_{\text {set. }}$ For these excitation parameters (rep. cycle $5 \mu \mathrm{s}$, pulse width $80 \mathrm{~ns}$, averaged excitation power $70 \mu \mathrm{W}$, focus diameter $20 \mu \mathrm{m}$ ) the proportion of $d l_{\text {SPPX }}$ to $I_{\text {set }}$ is $0.1 \%$.

In order to understand the differences between positive and negative bias voltages, we discuss the laser excitation at the GaAs surface (Fig. 2.13) in detail. Under dark conditions the tip induces at positive bias voltage a space charge region (SCR) with ionized donors at the surface (Fig. 2.13a). By applying optical excitation, electron and hole pairs will be generated (Fig. 2.13b). The tipinduced field separates this charge at the surface ${ }^{12,75-77}$. Photo-generated holes are attracted and accumulate at the surface. Due to the higher density of holes in comparison to the doping density, the tip-induced field is screened more efficiently leading to a drop of the band bending, also called a surface photovoltage (SPV) ${ }^{43}$. This results in an increase of the conduction band tunneling $I_{\mathrm{C}}$ and also leads to additional tunneling into the photo-generated holes via valence band tunneling $I_{\mathrm{V}}{ }^{17,18}$. At negative voltages and under dark conditions, we expect an inversion layer of the conduction band across the Fermi energy giving a confined electron gas at the surface (Fig. 2.13c). The additional generation of electrons causes only minor changes of the tunnel current (Fig. 2.13d). Hence, we attribute the signature in Fig 2.12f mainly to the thermal expansion of the tip. 


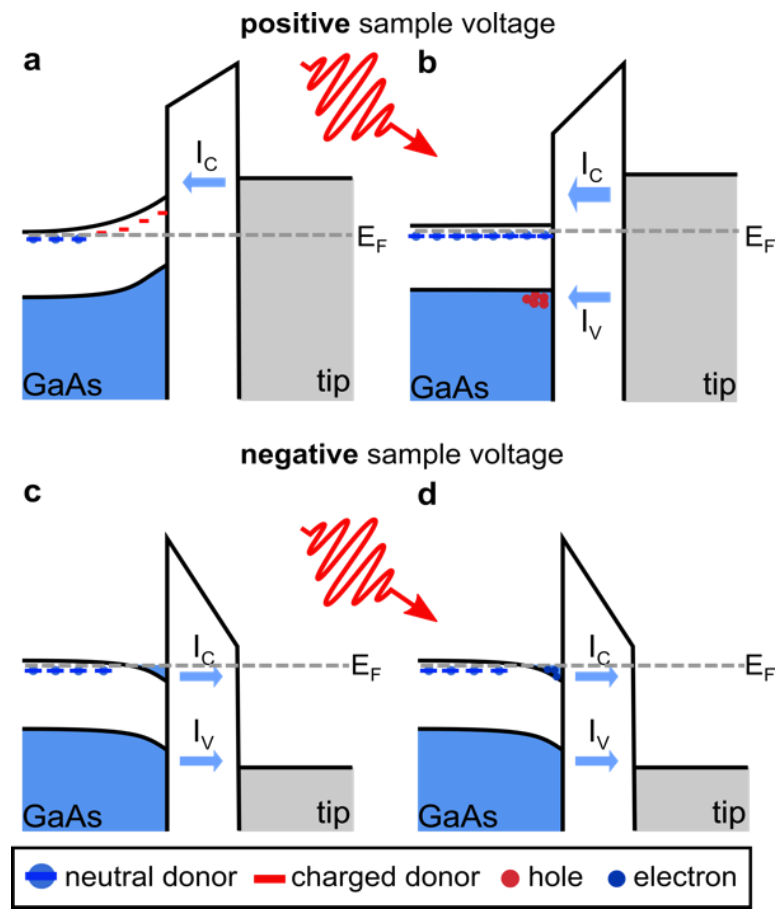

Fig. 2.13 Band scheme of GaAs with optical excitation for positive and negative sample voltage. (a, b) Optically induced processed at positive bias voltages: Optical excitation of the $E_{F}$ GaAs surface generates charge carriers, which are separated by the tip-induced field. Holes are accelerated towards the surface and accumulate in a small volume beneath the STM tip. The surface potential can be screened more effectively. The reduced band bending leads to more conduction band tunneling and additional valence band tunneling. (c, d) Optically induced processed at negative bias voltage: Already under dark conditions the electron gas in the conduction band, formed by an inversion layer, can screen the tip-induced potential quite efficiently. Although optical generated charge (in this case electrons) may move to the surface, the influence on the space charge region is weak. Optically induced changes in the tunnel current have mainly thermal origin.

To quantify thermal effects, we evaluated $d I_{\mathrm{SPPX}}$ at negative bias voltages as function of the set point currents $I_{\text {set. }}$. The laser focus is positioned slightly above the tip apex, i.e. position of the maximum signal (red box, Fig 2.12f). In this current range, $d I_{\text {SPPX }}$ increases linearly with $I_{\text {set }}$ (Fig 2.12g). We estimate the thermal contribution of $d I_{\mathrm{SPPX}}$ to $I_{\text {set }}$ to $0.1 \%$. It is important to note, that for these excitation parameters (rep. cycle $5 \mu \mathrm{s}$, pulse width $80 \mathrm{~ns}$, averaged excitation power $70 \mu \mathrm{W}$, focus diameter $20 \mu \mathrm{m})$ this is of the same order as the signal at positive voltages $(0.5 \%$, $d I_{\mathrm{SPPX}}=200 \mathrm{fA}$ to $I_{\text {set }}=50 \mathrm{pA}$ ). This demonstrates the requirement of an exact positioning of the laser focus at the tip apex. Otherwise thermal effects can superimpose with the electronic induced effects. As the maximum value of $d I_{\mathrm{SPPX}}$ at both bias voltages is spatially separated, the ratio between thermal and electronic effect can be estimated to 1:20 directly at the tip apex (red circle,

Fig 2.12e).

Fig. 2.14 Time spectra at the GaAs(110) surface on the atomic scale. $(\mathbf{a}, \mathbf{b}) d l_{\text {SPPx }}$ plotted against the delay time $t_{d}$ between pump and probe pulse for different positions on the GaAs surface. (c) STM topography of a single, buried dopant at the GaAs surface. (set point $0.8 \mathrm{~V}, 200 \mathrm{pA}$, exc. parameter: rep. cycle $8 \mu \mathrm{s}$, pulse width $40 \mathrm{~ns}$, averaged excitation power $12 \mu \mathrm{W}$, focus diameter $20 \mu \mathrm{m})$.

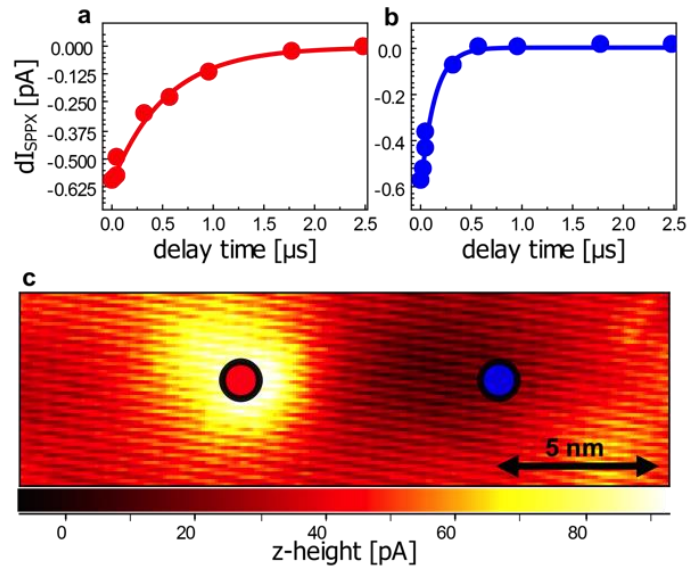


The ultimate goal of our method, i.e. combining optical excitation with atomically resolved STM operation, is demonstrated in Fig. 2.14. We have evaluated the relaxation dynamics of charge carriers near the GaAs(110) surface, given by the optical induced signal $d I_{\text {SPPX }}$ at positive bias voltages and as a function of the delay time $t_{\mathrm{d}}$, with atomic resolution. $d I_{\mathrm{SPPX}}\left(t_{\mathrm{d}}\right)$ spectra at different surface position are shown in Fig. 2.14a, b. Exponential fittings (solid line) determine the characteristic decay constant $\tau$. The variation in $\tau$, a topic beyond this review, can be explained by the local inhomogeneity at the GaAs surface, given by single buried dopants, visible in the simultaneously acquired STM topographies (Fig. 2.14c).

\section{Summary}

In conclusion we present a compact and versatile combination of optical excitation and STM. By generating the pulse sequence in an all-electronic way, parameters like pulse amplitude, width or repetition cycle can be easily adjusted to the experimental needs. We demonstrate this flexibility by studying dynamics at the optically excited $\operatorname{GaAs}(110)$ surface down to the single nanosecond time scale. By resolving processes at negative and positive bias voltages, thermally and electronically induced effects can be separated. The ratio between both signals can be estimated to 1:20. We resolved carrier dynamics at this surface. With the use of the atomic resolution of the STM, we are able to identify the influence of local defects on the relaxation process of the excited surface. For future experiments this promising synergy of pump-probe excitation and STM will promote the understanding of local dynamics at single defects, molecules or nanometer scaled interfaces considerably.

\subsection{General discussion}

As optical excitation in STM is far from being a standard method, existing technical implementations are diverse ${ }^{23-25,48,71,78}$. The presented setup in subchapter 2.2 bears a big advantage in contrast to sophisticated optical oscillators ${ }^{24,25}$. Excitation parameters like the pulse width, the repetition cycle or the pulse amplitude can be adjusted on the fly - for subtle changes even under tunneling conditions.

Thermal induced effects in STM operation has been widely discussed throughout the years but has not been quantified up to now. At the turn of the millennium the research group around Grafström et al. invested substantial effort in order to manage the thermal load during pulsed excitation at the STM tip ${ }^{22,51}$. Until then, already extensive results were obtained by using continuous wave excitation in STM in terms of the Surface Photovoltage (SPV) on the atomic scale ${ }^{11,60,79}$. However, Grafström et al. stated, that ultrafast STM is a promising experimental technique but thermal effects tend to mask the interesting processes. A renaissance that revived with the work of Shigekawa et al., in which they have resolved charge dynamics on multiple timescales. In their 
results, the outcome of Grafström et al. is referred but also it is claimed that at repetition rates of optical pulses greater than $10^{6} \mathrm{~Hz}$ thermal effects can be neglected because of the slower phonon response at these frequencies. Eventually, Dolocan et al. ${ }^{65}$ take up the doubts of Grafström et al. and disagree with Shigekawa et al. by proofing that thermal expansion and shrinking of the STM tip must as well be expected in the picosecond/femtosecond time domain. The results in this chapter show that both research groups are correct. For the first time, it is possible to quantify the thermal influence in STM experiments with pulsed optical excitation. The ratio between thermally induced current due to tip expansion effects and photo-induced current due to charge generation is estimated to 1:20. 



\section{Optical excitation at semiconductor surfaces in STM experiments}

This chapter discusses the method of SPV-STM. First, a brief introduction explains the common method of extracting a local Surface Photovoltage (SPV) in STM experiments. Then a short overview on previous results is given. In this subchapter also two exemplary publications are presented, showing the great strength of this method.

Finally, the role of the tunnel current in SPV-STM experiments is discussed. As this phenomenon has been mostly neglected up to now, our own studies show that the charge transfer via tunneling electrons will have a major impact on local surface potentials.

\subsection{How to extract the Surface Photovoltage in STM experiments}

In standard SPV experiments the change in the surface potential due to optical excitation is measured by determining the change in the surface capacity ${ }^{4,47}$. Instead, for SPV-STM, this value is determined by the change in bias voltage at a distinct tunnel current when applying optical excitation. Fig. 3.1 illustrates this effect of the SPV in the tunneling current for n-type GaAs. Under dark conditions (Fig. 3.1a), a depletion layer at the surface is found. Donor levels, positioned above the Fermi level, are ionized and thereby screen the tip potential, resulting in a SCR. The tunnel current into the $\mathrm{CB} I_{\mathrm{C}}$ is determined by voltage drop across the vacuum and the TIBB at the surface. Under optical excitation, the generated electron and holes are separated inside this SCR. Holes accumulate at the surface, screening the tip potential more efficiently. This leads to a drop of the TIBB (Fig. 3.1b). Consequently, at the same bias voltage, $I_{\mathrm{C}}$ increases, as the lack of the TIBB enables to address more states inside the $\mathrm{CB}$. At negative bias voltages (not shown), the inversion layer at the surface already accumulates electrons at the surface. Additional electron-hole pair generation induces only minor changes in the screening configuration for the tip potential and consequently no SPV is expected ${ }^{16}$.

Experimentally, this current increase is commonly accessed by comparing $I(V)$ spectroscopy for different optical excitation intensities. The research group of Hidemi Shigekawa ${ }^{12,16}$ chopped laser light at low frequencies while focusing the laser beam onto the tunnel junction *. An exemplary $I(V)$ spectra acquired with this method is shown in Fig. 3.1c. At positive bias voltages a periodically switching in the tunnel current can be observed. This splits the $I(V)$ spectra into two virtual curves: red dots with and blue dots without optical excitation. The horizontal comparison of both curves

\footnotetext{
${ }^{*}$ Interestingly, a variation in the tunnel current due to the periodic thermal expansion of the STM tip was not observed. In our case the application of this technique was impossible because of primary thermal effects.
} 
gives the voltage shift for a distinct tunnel current with and without optical excitation (Fig. 3.1c), which is interpreted as the SPV for this excitation intensity.

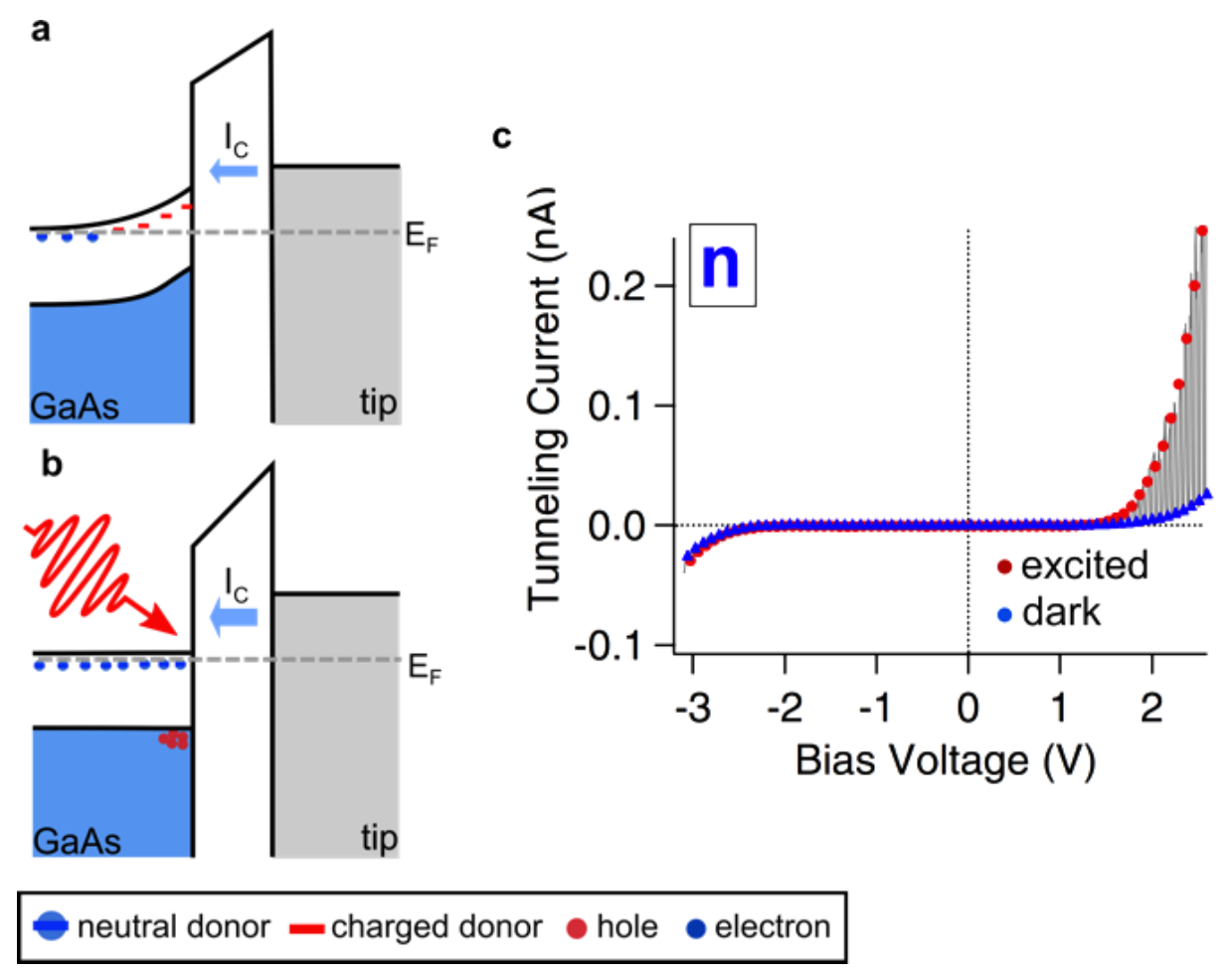

Fig. 3.1 How to measure the Surface Photo Voltage (SPV) with an STM. (a) Tunneling at the GaAs surface at positive bias voltages. (b) Optical excitation: Holes accumulate at the surface leading to a more efficient screening of the tip potential. The potential inside the surface drops, leading to an increase in conduction band tunneling. (c) $I(V)$ spectrum with chopped laser light (taken from ${ }^{16}$ ) at the n-doped GaAs surface gives two virtual $I(V)$ curves: one for the excited surface and one under dark conditions. The difference in bias voltage between both curves, resulting in the same tunnel current, is defined as the SPV.

\subsection{Preliminary studies of the local Surface Photovoltage}

Hamers et al. published the first studies of SPV-STM in the early nineties, mapping the SPV at the $7 \times 7$ reconstructed Silicon(111) surface, spatially resolved. The main result of this publication was the discovery of nanometer-sized areas having an increased carrier recombination rate, visible as a localized decrease in the SPV. Triggered by these promising results, various group pursued this idea and accomplished local SPV studies on a big variety of surfaces: $\mathrm{Ag}$ on $\mathrm{Si}(111)-7 \mathrm{x} 7^{10,12,60}$, $\mathrm{Si}(001)^{12,80}$, H-terminated $\mathrm{Si}(111)^{81}, \mathrm{Si}(111)-2 \times 1^{79}, \mathrm{Si}(111)-(5 \times 2)-\mathrm{Au}^{82}, \mathrm{WS}_{2}{ }^{13}, \mathrm{WSe}_{2}{ }^{54,83}$ and $\operatorname{GaAs}(110)^{11,16,24,52,64}$. In the following, two publications were summarized ${ }^{\dagger}$, investigating localized potentials variations at single defects or grown heterostructures by using of the SPV-STM. They show the possibilities of this rarely used method in order to map local potential modulations at semiconductor surfaces on the nanometer scale, extracted via the SPV.

\footnotetext{
${ }^{\dagger}$ These summaries do not include the whole content of the publications. Instead they focus on personal highlights of the studies, supporting the understanding of the presented thesis.
} 
Local Fermi-level pinning of a single As vacancy at the $\operatorname{GaAs(110)}$ surface $^{11}$

Originally by S. Aloni, I. Nevo and G. Haase, published in PRB 60, R2165(R) (1999).

In this study, Aloni et al. used the lack of surface or defect states at a clean GaAs(110) surface (ptype) in order to determine the induced potential and the local Fermi-level pinning of Arsenic (As) vacancies. Fig. 3.2a shows a constant current topography of this vacancy and in Fig. 3.2b the corresponding map of the local SPV can be seen. In both cases the signature of the As vacancy is visible as a localized depletion.

They used chopped, super-band-gap illumination in order to estimate the SPV, analogue to Fig. 3.1c. Additionally, it is assumed that VB and CB are flattened during excitation as a result of the low surface recombination rate for $\operatorname{GaAs}(110)$ and large carrier diffusion length ${ }^{46}$. This assumption and the lack of band gap states allow interpreting the SPV as pure TIBB or in presence of local defects as the superposition of TIBB and defect potential.
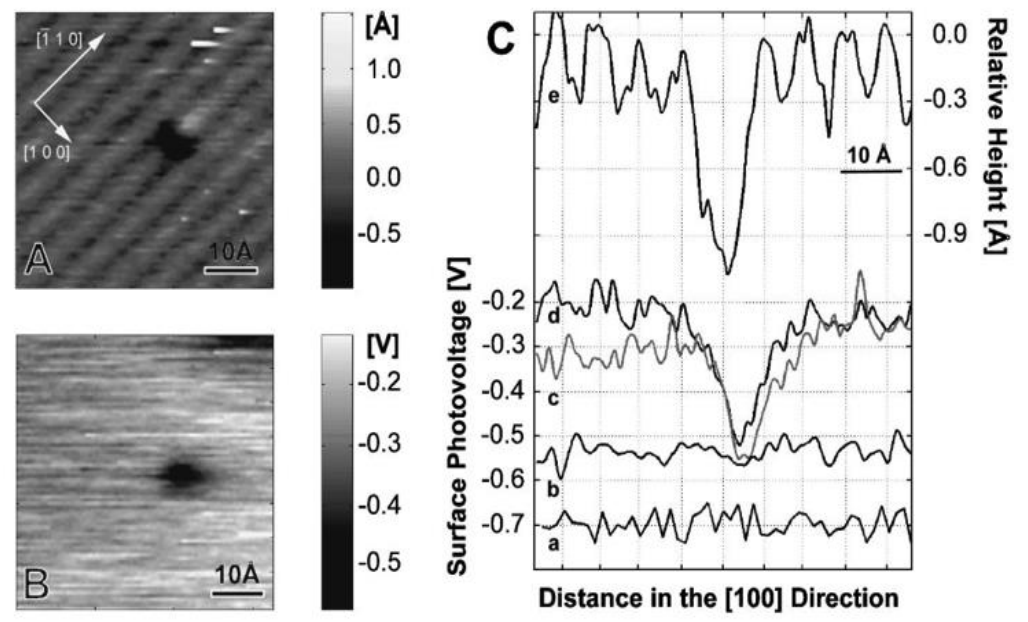

Fig. 3.2 Local Fermi-level pinning at an As vacancy at the p-doped GaAs(110) surface, probed by SPV-STM. (a) Atomically resolved As vacancy. (b) Simultaneously recorded, locally resolved SPV image (-2.8 V, $25 \mathrm{pA})$. (c) Sections along the topographic image in (a) and along the SPV image in (b) for different bias voltages. SPV a: -3.5 V 29pA, b: -3 V 18pA, c: $-2.5 \mathrm{~V} 29 \mathrm{pA}, \mathrm{d}:-1.8 \mathrm{~V}$ 30 pA. Topography e: $-2.8 \vee 25$ pA. Taken from ${ }^{11}$.

Fig. 3.2c shows line profiles of the SPV taken across the As vacancy for different bias voltages (ad). For reference, also a corresponding topographic profile, taken from Fig. 3.2a, is included (e). Beginning with high bias voltages, e.g. $-3.5 \mathrm{~V}$ (a), the SPV is solely determined by the TIBB. Far away from the defect, as expected, the SPV decreases with decreasing bias voltages. Interestingly, (c), a local depletion is found at the As vacancy in comparison to the free surface at $-2.5 \mathrm{~V}$. By decreasing the bias voltage even more, the absolute value of this local minimum remains constant.

\footnotetext{
\$ Please note, that, in case of p-type GaAs, the depleting potential at the surface, visible as band bending in VB and CB, is given for negative bias voltages.
} 
The depletion at this voltage and especially the independency on the tip potential for lower voltages is interpreted as local Fermi-level pinning induced by the defect.

Microscopic Basis for the Mechanism of Carrier Dynamics in an Operating pn Junction Examined by Using Light-Modulated Scanning Tunneling Spectroscopy ${ }^{16}$

Originally by Yoshida et al., published in PRL 98, 026802 (2007)

Local electric fields play a major role in every applied semiconductor services. Macroscopically not tangible, Yoshida et al. used the SPV-STM to map the nano-scaled potential of an optical excited pin-junction made of GaAs. Fig. 3.3a shows a sketch of the experimental setup. Opticalexcited charge is not only influenced by the tip-induced field but also senses the intrinsic local potential at the junction, leading to a lateral charge separation towards the n-type or p-type doping region, respectively. Additionally, it is possible to apply a transverse voltage and drive a current through the junction.
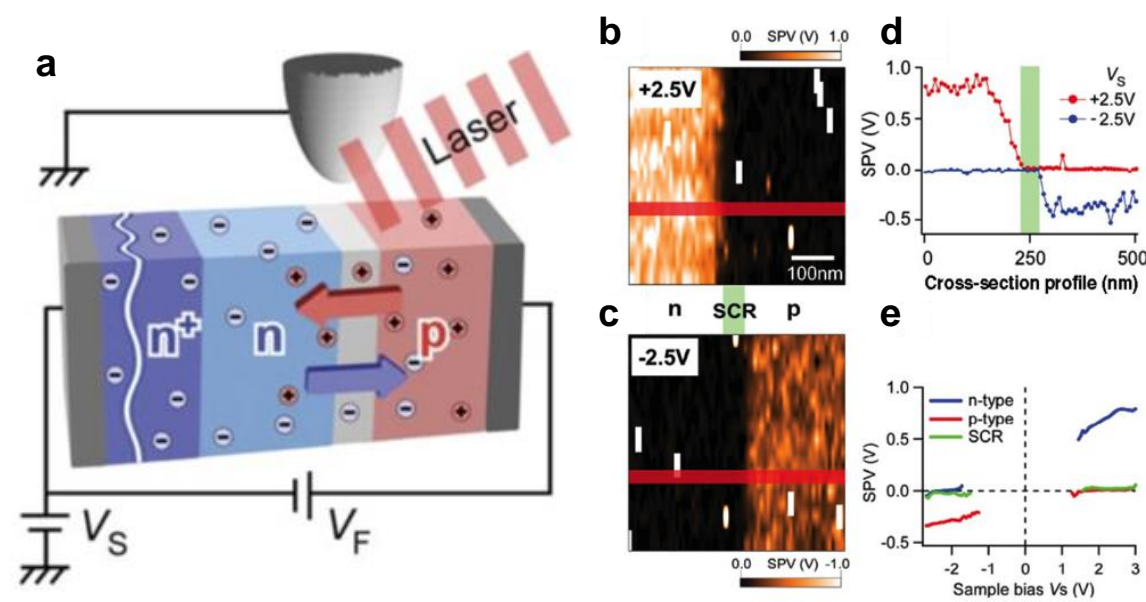

Fig. 3.3 Local mapping of the surface potential at a pin-junction, cross-sectional resolved via SPV-STM. (a) Experimental setup. (b, c) Map of the SPV at the junction for positive and negative voltages. (d) Correspondent sections of the SPV across the junction. (e) Voltage dependency of the SPV for the n- and p-type region and at the SCR of the pinjunction. Taken from ${ }^{16}$.

Locally resolved SPV for positive and negative bias voltages across the pin-junction are shown in Fig. 3.3b, c. As expected, in the n-doped (p-doped) region and far away from the pin-interface the SPV corresponds to the locally induced TIBB for positive (negative) voltages. Fig. 3.3d shows sections along the junction. In the presence of the SCR at the junction the SPV decreases, whereas it completely vanishes in the intrinsic area (green shade). Fig. 3.3e summarizes the voltage dependency of the SPV far from the junction and in the intrinsic region. 
a

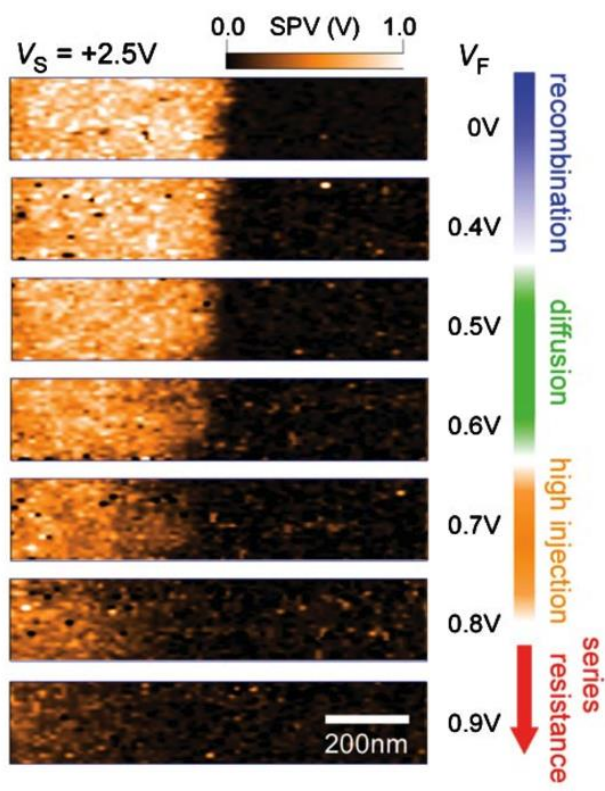

b

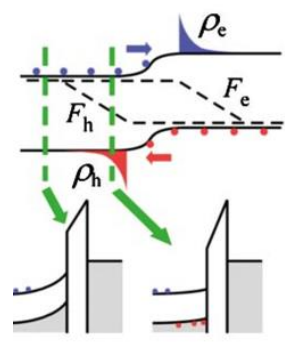

C

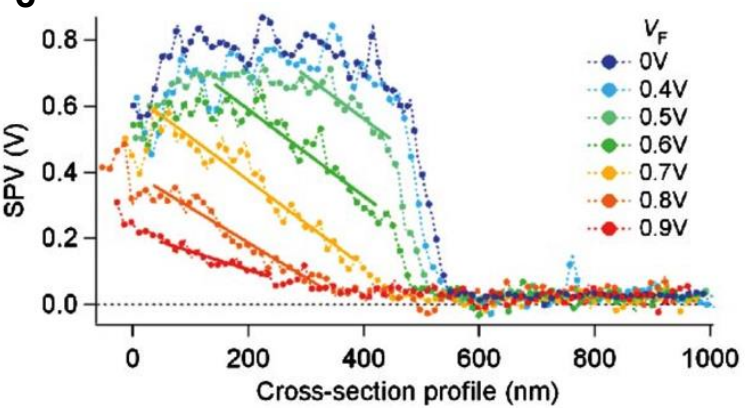

Fig. 3.4 Change of the SPV for positive bias voltages when driving a transversal current across the junction. (a) SPV map at the pn-junction for different transversal current densities. (b) TIBB at the surface when carriers are injected by the transversal current. (c) Sections of the SPV across the junction show the influence of the transversal current. Taken from ${ }^{16}$.

By driving a current through the pin-junction, additionally to the optical excited charge carriers, minority carriers can be found in the correspondent n- and p-type region (Fig. 3.4b). Consequently, depending on the magnitude of the transversal voltage $V_{\mathrm{F}}$, the potentials in the presence of the pinjunction change considerably, visible in local maps of the SPV at positive bias voltages and different current densities across the junction (Fig. 3.4a). At $2.5 \mathrm{~V}$ and zero transversal current the SPV map is identical to Fig. 3.3b. The magnitude of SPV decreases for increasing current densities through the junction.

More prominent is the size of the depletion area of SPV in the presence of the pin-junction, which increases when increasing the transversal current (Fig. 3.4c). According to Yoshida et al. this is connected to different transport regimes of the pin-junction, modifying the size of the SCR considerably and thereby weakening the effect of a SPV.

\subsection{Controlling the screening process of a space charge region by minority carriers}

In nearly all-local SPV studies, the STM is treated as a semi non-invasive method. On one hand, it is stated that the tip potential penetrating into the substrate surface has a significant influence on the SPV. On the other hand, tunneling electrons and thereby the charge transfer into the sample is neglected when evaluating the SPV. Although, early studies already touched this subject ${ }^{13,17,79}$ showing clear indications of an influence of the tunnel current on the SPV, it is given virtually no 
compliance in the literature. In the following subchapter a detailed study on the interplay between charge generation via optical excitation and charge annihilation via the tunnel current is presented.

This subchapter is published in identical words in the scientific journal "Nature Communications". The experiments were conducted in collaboration with Katharina Kaiser. The manuscript was written together with Martin Wenderoth. Parts of the results can also be found in the bachelor thesis of Katharina Kaiser (University of Göttingen, 2014). For the sacrifice of the readability of this thesis, the next paragraph gives a short summary. Additionally the results of this publication and its impact on the subject "studying SPV with the STM" are discussed at the end of this chapter. This subchapter contains the main manuscript and the additional supplementary information (abbreviated with S3.1 - S3.6).

\section{Brief summary}

We are able to directly proof, that photo-generated holes not only change the screening length of the tip-induced surface potential (build-up of a SPV) but can also actively contribute to the tunneling process. A characteristic trademark of the GaAs(110) surface is the distinct visible atomic corrugation in constant-current topographies, depending which final state in the tunnel process is addressed. At low positive sample voltages it is possible to acquire the atomic corrugation, characteristic for tunneling at negative voltages under dark conditions. Photogenerated holes, positioned energetically inside the valence band, are contributing to the tunnel process.

For a detailed analysis of this interplay between photo-generated charge and valence band tunneling we performed $I(z)$ spectroscopies at different bias voltages and optical excitation densities. Under dark conditions the tunnel current $I$ follows an expected exponential dependency when varying the tip height $z$. With optical excitation the run of the $I(z)$ curves changes considerably. For a distinct tunnel current a plateau region emerges which moves to higher currents when increasing bias voltage or optical intensity. With this plateau region we are able to divide the $I(z)$ spectra in three different regimes, whereby, in the first and third regime an exponential dependency between $I$ and $z$ is still given.

We explain the three regimes with different steady states between hole generation and hole annihilation. In the first regime, small tunnel currents do not change the density of the photogenerated hole gas. This density is determined by an equilibrium between the hole-generation rate and the intrinsic recombination rate. A preliminary work has shown that even at low intensities socalled flat band conditions are given. Increasing the current (moving the tip towards the surface), at the plateau region the steady state of hole generation and annihilation is distorted. In this regime the tunnel current actively changes the hole concentration. In order to replace the missing charge, formerly surface-near, neutral dopants recharge and the tip-induced potential rebuilds, leading to a 
decrease of the tunnel current. At the crossover to the last regime, the $I(z)$ characteristics become exponential again. All photo-generated holes at the surface are instantly annihilated by tunneling electrons and the tip-induced potential is identical to the one without optical excitation. Applying a simple tunneling model we are able to extract parameters like the diffusional or field-driven capture rate of holes inside the space charge region.

A closer look on the spectra reveals a significant change in the noise characteristics in the tunnel current at the crossover points before and after the plateau region. Already preliminary studies have shown that continuous charging and discharging of donors can induce current fluctuations under dark conditions ${ }^{19,84}$. The comparison with $I(z)$ spectra without optical excitation reveals that the presence of photo-generated charge can influence the noise level considerably. Whereas in the plateau region the noise may enhance, it significantly decreases in the third regime of the $I(z)$ spectra.

\section{Original publication}

The miniaturization of future electronic devices is intimately connected to the ability to control electric fields on the atomic scale. In a nanoscopic system defined by a limited number of charges, the combined dynamics of bound and free charges become important. Here we present a model system based on the electrostatic interaction between a metallic tip of a Scanning Tunneling Microscope and a GaAs(110) semiconductor surface. The system is driven out of equilibrium by optical excitation providing ambipolar free charge carriers as well as by an optically induced unipolar tunnel current. This combination allows to actively control the density and spatial distribution of free and bound charge in the space charge region, i.e. modifying the screening processes. Temporal fluctuations of single dopants are modified, i.e. we are able to control the noise of the system. It is found that free charge carriers suppress the noise level in field-controlled, nanoscopic systems.

P. Kloth, K. Kaiser and M. Wenderoth, Nat. Com. 7, 10108 (2016), DOI: 10.1038/ncomms10108

\section{Introduction}

Nowadays semiconductor based electronic devices have already reached the nanometer size $\mathrm{e}^{1,85,86}$. At this scale the discreteness of charge, given by e.g. charged single impurities, has already become visible ${ }^{19,87}$. The latter are the natural limit of the miniaturization process of future electronic applications. Processes, which define the equilibrium charge state of such a nanoscopic device, are shown in Fig. 3.5a. (i) A gate voltage ionizes dopant atoms in order to build up the local space charge region (SCR). (ii) A current of free minority charge carriers $I_{\mathrm{H}}$ towards the field region sets in (Fig. 3.5a, $\alpha$ ). (iii) Recombination of carriers inside the SCR results in spatial and temporal fluctuating charges. All this depends on the charge density, the emission (Fig. 3.5a, $\beta$ ) and capture rate (Fig. 3.5a, $\delta$ ) of dopants and the dynamics of the minority charge carriers. 
In our approach the sharp tip of a Scanning Tunneling Microscope (STM) serves as the very local gate $^{19,87-92}$. Free charge generation by optical excitation and carrier injection by the tunnel current allows driving the system out of equilibrium. We are able to actively control the charge configuration at the surface and thereby establish different screening processes. Values like the carrier flow inside the SCR for different tunnel currents, optical excitation powers and bias voltages can be extracted. Moreover, it is possible to characterize the charge dynamics by using a noise analysis of the tunnel current for different charge configurations at the surface.
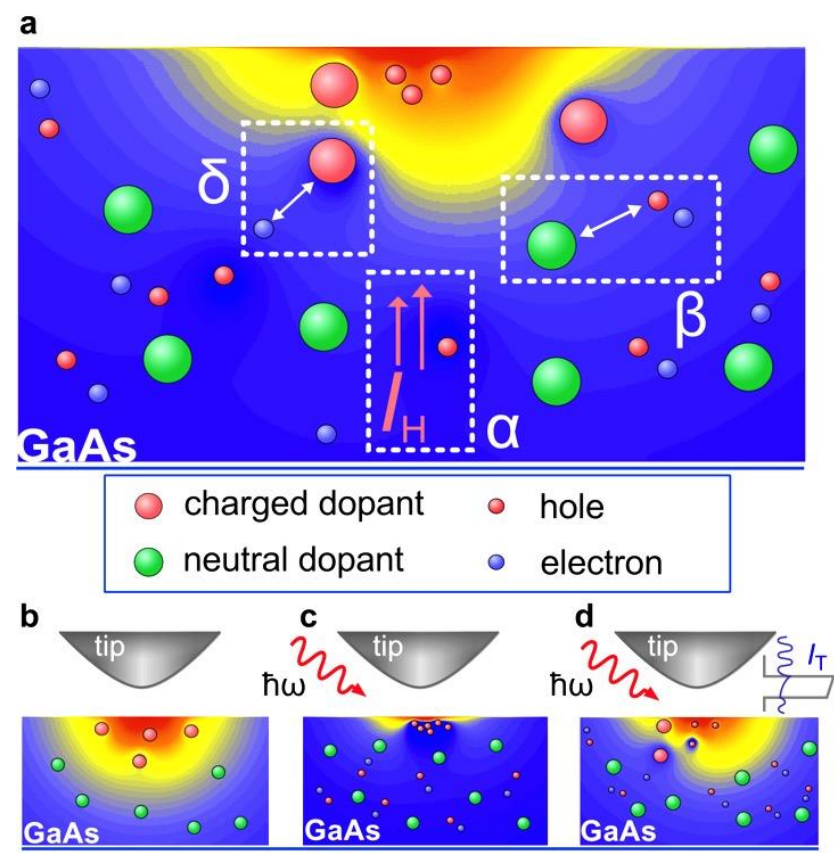

Fig. 3.5 Charge dynamics inside a localized space charge region (SCR) (a) The geometry and spatial extend of a SCR is very sensitive to the charge distribution. Besides ionized dopants, a current of minority charge carriers $I_{H}$ towards the surface is found ( $\alpha$ ). This charge replaces the function of the ionized dopants. Doping atoms charge $(\beta)$ and discharge $(\delta)$ by capturing free holes or electrons depending on the hole density at the surface. (b) The STM-tip-induced potential is used as a local gate electrode to create a SCR of ionized dopants reaching several nanometers into the sample. (c) By generating photo-excited electrons and holes, the free charge is separated by the tip-induced field resulting in a hole accumulation at the surface and thereby to a more efficient screening of the potential drop. (d) Tunneling electrons address the hole gas, driving the system out of its equilibrium state. By changing the tunnel current, the hole density and, thus, the screening length of the SCR is controlled.

\section{STM on the GaAs(110) surface under optical excitation}

Positioned only a few Ångstroms above a surface with a low carrier density, the metallic tip induces a nanoscopic SCR inside the sample (Fig. 3.5b). At an n-doped $\left(3 \cdot 10^{18} / \mathrm{cm}^{3}\right) \mathrm{GaAs}(110)$ surface with a positive sample voltage of $2.5 \mathrm{~V}$ applied, a depleting charge layer is induced extending $\sim 15 \mathrm{~nm}$ into the sample surface with only $\sim 10$ ionized dopants involved ${ }^{4}$. To get access to the dynamics of this nanoscaled system, we have combined STM with optical excitation (Fig. 3.5c). Photo-generated electron-hole pairs will be separated by the electric field. In equilibrium, this current of positive minority charge carriers $I_{\mathrm{H}}$ (n-doped) towards the surface is balanced by recombination and thermionic emission. The SCR is modified by the hole accumulation, screening the potential between tip and sample more efficiently. Originally ionized dopants partly discharge, which results in a shift of the surface potential, by default described as a surface photovoltage ${ }^{3}$ (SPV). 
The photo-generated charge accumulation at the surface results in an additional current channel $^{13,17,54,67}$. Carrier injection via the tunnel current $I_{\mathrm{T}}$ into the valence band distorts the balance of free charge given by optical excitation and locally fixed charge of ionized dopants inside the SCR (Fig. 3.5d), modifying the non-equilibrium conditions. Surprisingly within the framework of locally resolved SPV experiments, this carrier injection has been widely neglected up to now ${ }^{11,12,60}$. The influence of $I_{\mathrm{T}}$ on the screening process of the SCR has been subject to studies before ${ }^{13,25,79,93}$. Cahill et al. ${ }^{79}$ describes this effect as a consequence of a "charging" due to high tunnel currents leading to an electron accumulation at the surface and counteracting the photo-generated holes. Similar observations were made by Terada et al. ${ }^{25}$ discussed as an increase of hole recombination at increased tunnel currents. Chen et al. ${ }^{93}$ describe the change in the SCR as a result of a leakage current modifying the concentration of the free, photo-generated charge and is treated as a parasitic side effect. More sophisticated is the work of Sommerhalter et $\mathrm{al}^{13}$. By observing direct tunneling into minority carriers they succeeded to develop a model considering the influence of the charge injection by $I_{\mathrm{T}}$.

In our experiment $I_{\mathrm{T}}$ is used as a control parameter for the charge distribution inside the SCR. Unlike previous studies we keep the tip-sample potential constant when changing the tunnel current. This allows us to study the balance of free and bound charge at the surface by actively tuning the screening process of the SCR and to disentangle and control the dynamics of the system by only changing $I_{\mathrm{T}}$. At weak photo-illumination we are able to investigate the full range of carrier injection going from low to high currents and thereby at the same time testing and modifying the screening process in three regimes.

\section{Valence band tunneling visible in STM topographies}

In Fig. 3.6a, b constant current topographies of the optical-excited $\mathrm{GaAs}(110)$ for positive bias voltages visualize the contribution of tunneling into photo-generated minority carriers for the first time locally resolved. Different atomic corrugations are observed, which can be attributed to specific resonant surface states positioned energetically inside the valence band (Fig. 3.6a) and conduction band (Fig. 3.6b) ) $^{33,34}$ (see section S3.1 and Fig. 3.8). The visibility of the valence band corrugation (Fig. 3.6a) at positive bias voltage is, in contrast to spectroscopic measurements, direct evidence that tunneling into photo-generated holes via valence band tunneling $I_{\mathrm{V}}$ is possible. Under dark conditions at $0.3 \mathrm{~V}$ bias voltage the conduction band cannot be addressed due to the tip induced band bending. With optical excitation it is a priori open which tunnel channel (valence or conduction band) is dominant and depends strongly on the density of states and the transmission probability (for details see section S3.2). 

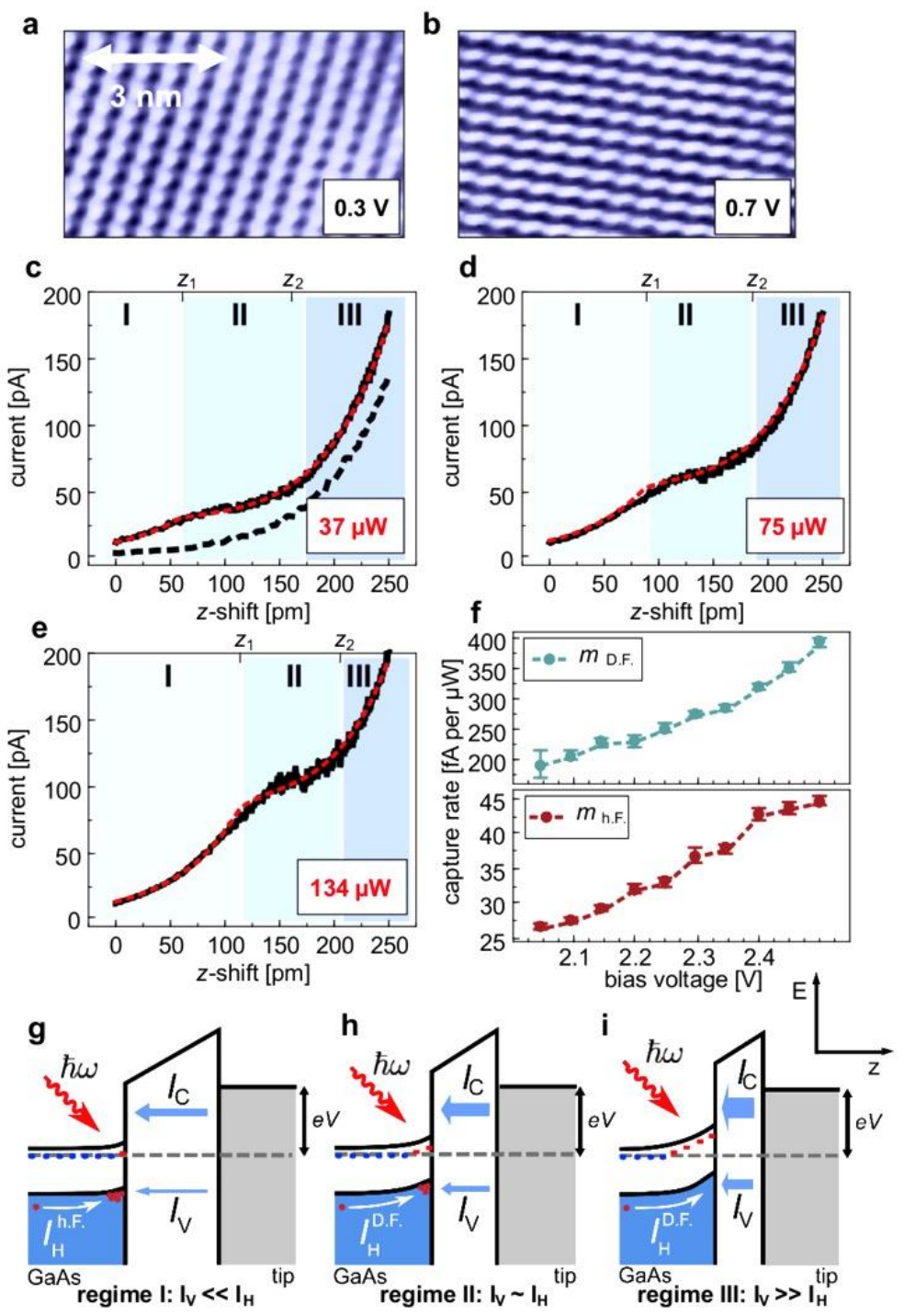

Fig. 3.6 Constant current topographies (CCT), I(z) spectra and a two-channel model discussing the interplay of the charge inside the SCR. (a) CCT at $0.3 \mathrm{~V}(400 \mathrm{pA})$ shows the atomic corrugation characteristic for valence band tunneling. (b) At $0.7 \mathrm{~V}(400 \mathrm{pA})$ the corrugation stemming from conduction band states is visible. (c-e) Optical excitation dependent $I(z)$ spectroscopies (set point: $2.5 \mathrm{~V} / 10 \mathrm{pA}$ ). A plateau appears in the curves, which moves in position for increasing optical power. The black dashed line shows the $I(z)$ characteristics without optical excitation. (f) Hole field induced $m_{\text {h.F. }}$ and dopant field induced $m_{\text {D.F. }}$ capture rate of holes at the surface. (g-i) A model including both tunnel channels attributes different charge configurations to the three regimes in the $I(z)$ characteristics. Model calculations are shown as red-dotted lines in (c-e).

\section{Accessing different regimes of valence band tunneling with $I(z)$ spectroscopy}

By using $I(z)$ spectroscopy as a function of low optical excitation power $P_{\mathrm{L}}$ and at fixed bias voltage we are able to exclusively monitor the influence of $I_{\mathrm{T}}$ on the screening process inside the surface. The change in the surface potential when decreasing the tip-sample distance by $3 \AA$ is about $5 \%$ of the whole SCR and hence is negligible. Fig. 3.6c-e shows a set of $I(z)$ curves taken at a set point of $2.5 \mathrm{~V}$ and $10 \mathrm{pA}$. It is important to note that this set point allows to directly compare 
the curves acquired at different $P_{\mathrm{L}}$ (see section S.3.1 and Fig. 3.9). A plateau-like signature can be identified in the experimental data, moving in position when changing $P_{\mathrm{L}}$ and bias voltage (the latter not shown here). In standard tunnel theory the current dependency $I(z)$ on the tip sample distance $z$ is described by an exponential relation $I(z) \propto \exp (-2 \alpha z)$ with $\alpha=\sqrt{(} 2 m \Phi) / \hbar$ and $\Phi$ as the apparent barrier height of the tunnel junction ${ }^{94}$.

A closer look on the $I(z)$ spectra in Fig. 3.6c-e strongly suggests to divide the curve into three electronic configurations, indicated by the blue shadings and labeled as I, II and III. In I and III the $I(z)$ curves follow the expected exponential dependency. We extract a barrier height $\Phi$ for the tunnelling electrons of $2.5 \mathrm{eV}$ (see section S3.3 and Fig. 3.10), the same value obtained for the $I(z)$ characteristics under dark conditions (dotted line in Fig. 3.6c). Analogous to the topographic analysis we conclude that conduction band tunnelling is the predominant process at higher bias voltages. Nevertheless, the topography in Fig. 3.6a demonstrates that the tunnelling into photogenerated holes is possible.

\section{Modeling the tunneling into photo-generated minority carriers}

In order to describe the complex $I(z)$ characteristics under optical excitation we present a twochannel tunneling model (Fig. 3.6g-i), which includes both, the current into the valence band $I_{V}$ and into the conduction band $\mathrm{I}_{\mathrm{C}}$.

In I (Fig. 3.6e) the valence band tunneling $I_{\mathrm{V}}$ is as low, that it is not significantly influencing the charge density $n_{\mathrm{H}}$ at the surface. The electrostatic potential between tip and sample is completely screened by the photo-generated charge, leading to flat band conditions for all optical power ratings $P_{\mathrm{L}}$. The tunneling into the valence band $I_{\mathrm{V}}$ is balanced by a hole current $I_{\mathrm{H}}^{\text {h.F. }}$. This current can be regarded as a field driven current $I_{\mathrm{H}}^{\text {h.F. }}$, induced by the distortion of the hole gas screening process (hole gas induced Field: h.F., see section S3.4 and Fig. 3.11). In this regime, the transmission probability and not the density of final states of the tunneling process is the limiting factor for valence band tunneling $I_{\mathrm{V}}$. Therefore, $n_{\mathrm{H}}$ defined by the potential between tip and sample and also the SCR is constant as a function of the tunnel current $I_{\mathrm{T}}$.

For higher currents, $I_{\mathrm{V}}$ overcomes $I_{\mathrm{H}}^{\text {h.F. }}$, resulting in a filling of final states for tunneling electrons. Consequently, the hole concentration at the surface decreases (Fig. 3.6h). At the crossover from I to II (at $z_{1}$ ) valence band tunneling $I_{\mathrm{V}}$ and the hole current $I_{\mathrm{H}}^{\text {h.F. }}$ have equalized. In this transition regime II the hole density $n_{\mathrm{H}}$ can be actively tuned by adjusting the tunnel current. As this value defines the screening length of the induced field, the controlled decrease of $n_{\mathrm{H}}$ at the surface leads to a $I_{\mathrm{V}}$ dependent rebuilding of the SCR (Fig. 3.6h). As a consequence two counteracting processes set in. First, due to the rebuilding of the SCR the conduction band tunneling $I_{\mathrm{C}}$ decreases, as a fraction of the bias voltage drops inside the sample. Secondly, the longer spatial extend of the resulting SCR changes the current of photo-generated holes towards the surface. The holes are accelerated by the tip-induced field, partly screened by ionized dopants, resulting in a more 
pronounced field driven hole current $I_{\mathrm{H}}^{\text {D.F. }}$ (Dopant induced Field: D.F., see section S3.5 and Fig. 3.15). Consequently, $I_{\mathrm{H}}$ and as a result the valence band tunneling $I_{\mathrm{V}}$ increases. Nevertheless, the plateau in the $I(z)$ curves in II shows that the decrease of the conduction band tunneling due to the change of the SCR is the dominant effect. At the end of regime II (at $\left.z_{2}\right)$ the SCR found without optical excitation is re-established.

Regime III (Fig. 3.6i) is defined by the lowest hole density at the surface $\left(n_{\mathrm{H}} \approx 0\right)$. The tunneling into the valence band has even exceeded $I_{\mathrm{H}}^{\text {D.F. }}$. This corresponds to an instant annihilation of all holes participating in the screening process of the potential between tip and sample. The charge is solely provided by ionized, locally fixed dopants. The tunneling current $I(z)$ can be described by the sum of the exponential $I_{\mathrm{C}}(z)$ characteristics of conduction band tunneling plus the valence band tunneling $I_{\mathrm{V}}$ balancing the field-driven current of holes towards the surface. Further increase of the tunneling current $I_{\mathrm{T}}$ does not change $\mathrm{n}_{\mathrm{H}}$ and thereby the SCR. $I_{\mathrm{V}}$, being limited by $I_{\mathrm{H}}^{\text {D.F. }}$, becomes a constant, tunnel current independent contribution, only dependent on the bias voltage.

In order to quantify this model, we describe the overall current $I(z)$ as the sum of valence band $I_{\mathrm{V}}(z)$ and conduction band tunneling $I_{\mathrm{C}}(z)$ in the three different current regimes. Our model is able to accurately reproduce the observation (red lines in Fig. 3.6c-e, see section S3.6). A detailed analysis (see section S3.4 and S3.5) shows that both, the hole gas induced field driven current $I_{\mathrm{H}}^{\text {h.F. }}$ and the dopant induced field driven current $I_{\mathrm{H}}^{\text {D.F. }}$, depend linearly on the optical excitation power $P_{\mathrm{L}}$ $\left(I_{\mathrm{H}}^{\text {h.F. }}=m_{\text {h.F. }} \cdot P_{\mathrm{L}}\right.$ and $\left.I_{\mathrm{H}}^{\text {D.F. }}=m_{\text {D.F. }} \cdot P_{\mathrm{L}}\right)$. According to the model at $z_{1}$ or $z_{2}$ the valence band tunneling has equalized $I_{\mathrm{H}}^{\text {h.F. }}$ or $I_{\mathrm{H}}^{\text {D.F. }}$, respectively, allowing us to extract the charge generation inside the SCR. The capture rate $m_{\text {D.F. }}\left(\mathbf{z}_{2}\right)$ is 8 to 9 times higher in comparison to $m_{\text {h.F. }}$ (Fig. 3.6f). The model allows us to calculate and to separate valence and conduction band tunneling (see section S3.6). At an optical power of $100 \mu \mathrm{W}$ valence band tunneling contributes $4 \%$ at $z_{1}$, whereas at $z_{2}$ the valence band tunneling accounts for over $30 \%$ of the overall tunnel current. The latter corresponds to an annihilation of $2.5 \cdot 10^{8}$ holes per second. With a focus diameter of $50 \mu \mathrm{m}$ and a penetration depth of about $1 \mu \mathrm{m}$ of the laser light ${ }^{95}$, holes in an excitation volume of roughly $(40 \mathrm{~nm})^{3}$ are collected. This volume has the same magnitude as the spatial extends of the SCR under dark conditions, suggesting that in regime III all holes in the tip-induced electric field are depopulated. Both values, $m_{\text {h.F. }}$ and $m_{\text {D.F. }}$, show a significant bias voltage dependency, which is correlated to the potential drop between STM tip and sample that is needed to be screened (for more details see section S3.4 and S3.5).

In order to sum up this section we compare our results and the model with previous studies ${ }^{13,25,79,93}$. Contrary to ref. ${ }^{25,79}$ we can exclude the effect of increased electron injection into the conduction band for high tunnel currents leading to a change in the SCR, as the topographic analysis in Fig. 3.6a,b is able to clearly show additional valence band tunneling into minority carriers. This mechanism, also discussed in ${ }^{13,93}$, reacts very sensitive to the size of the SCR, dependent on the STM tip geometry, the potential between tip and sample and the density of optical excitation. By 
keeping the bias voltage fixed and applying low optical excitation the $I(z)$ spectroscopy allows us to develop a quantitative two channel tunneling model. An advantage of our experimental conditions in comparison to previous work ${ }^{13}$ is the combination of conduction band $I_{\mathrm{C}}$ and valence band tunneling $I_{\mathrm{V}}$ enabling us to monitor the change of the SCR, having a major influence on $I_{\mathrm{C}}$, and the behavior of the tunneling into photo-generated minority carriers, giving the position of the plateau region in the $I(z)$ curves, simultaneously. Also by having a defined SCR in regime I (flat band conditions) and III (TIBB under dark conditions) an extensive computational effort of the tipinduced potential can be avoided.

\section{Noise analysis of the nanoscaled Space Charge Region}

Concluding, it is obvious that different charge configurations have to be considered in the discussed three regimes. In order to extract the corresponding dynamic properties we analyze the noise characteristics of the tunnel current. By subtracting a low frequency filtered $I(z)$ curve from the raw data, the signal in a frequency range between $10 \mathrm{~Hz}$ and $1.5 \mathrm{kHz}$ is isolated (Fig. 3.7a). In comparison to the noise without optical excitation we observe a spontaneous increase in noise in regime II in spectra taken at the photo-excited surface. The standard deviation $\sigma_{\mathrm{RMS}}$ in current is plotted against the average current for an optical excitation of $37 \mu \mathrm{W}, 75 \mu \mathrm{W}$ and $134 \mu \mathrm{W}$ compared to the values under dark conditions in Fig. 3.7b-d. The behavior of the noise level in the excited case is unequivocally connected to the previously discussed tunneling regimes, indicated by the blue shadings in Fig. 3.7b-d.

At $37 \mu \mathrm{W}$ optical excitation (Fig. 3.7b) the noise in the tunnel regime I shows the same trend compared to the non-excited case. For regime II, $\sigma_{\text {RMS }}$ increases. We attribute this to a nonequilibrium charging and discharging process of dopants ${ }^{19,87,91,96}$ inside the SCR due to the carrier injection. By disturbing the hole density at the surface by valence band tunnelling $I_{\mathrm{V}}$ drives the SCR in metastable configurations. We expect subtle but frequent changes in the geometry of the SCR leading to variations in the tunnelling current, and thereby to additional noise.

In regime III we observe a saturation of $\sigma_{\mathrm{RMS}}$, yielding $\sigma_{\mathrm{RMS}}(37 \mu \mathrm{W})<\sigma_{\mathrm{RMS}}(0)$. Previous studies ${ }^{19,84}$ have shown that under dark conditions bistable charging processes of doping atoms have to be taken into account. Assuming that these processes define the noise level of the spectra without optical excitation, our results suggest that the presence of free electron-hole pairs suppresses this charge switching in the photo-excited case ${ }^{9,26}$. We like to point out that the decrease in noise in comparison to the noise under dark conditions is not the result of a simple decrease of the SCR. Our analysis suggests that in regime III the SCR, normally found under dark conditions, has been rebuilt. Here the charge of the SCR is solely defined by ionized dopants. But even though no more hole accumulation is expected at the surface in the tip-induced field, it is conceivable that the electron-hole pairs, present in the bulk material, cause an additional screening of the SCR. The 
geometry of the SCR, normally given by the rather stochastic distribution of donors, is smoothed and thereby the charge state of the dopants is stabilized.
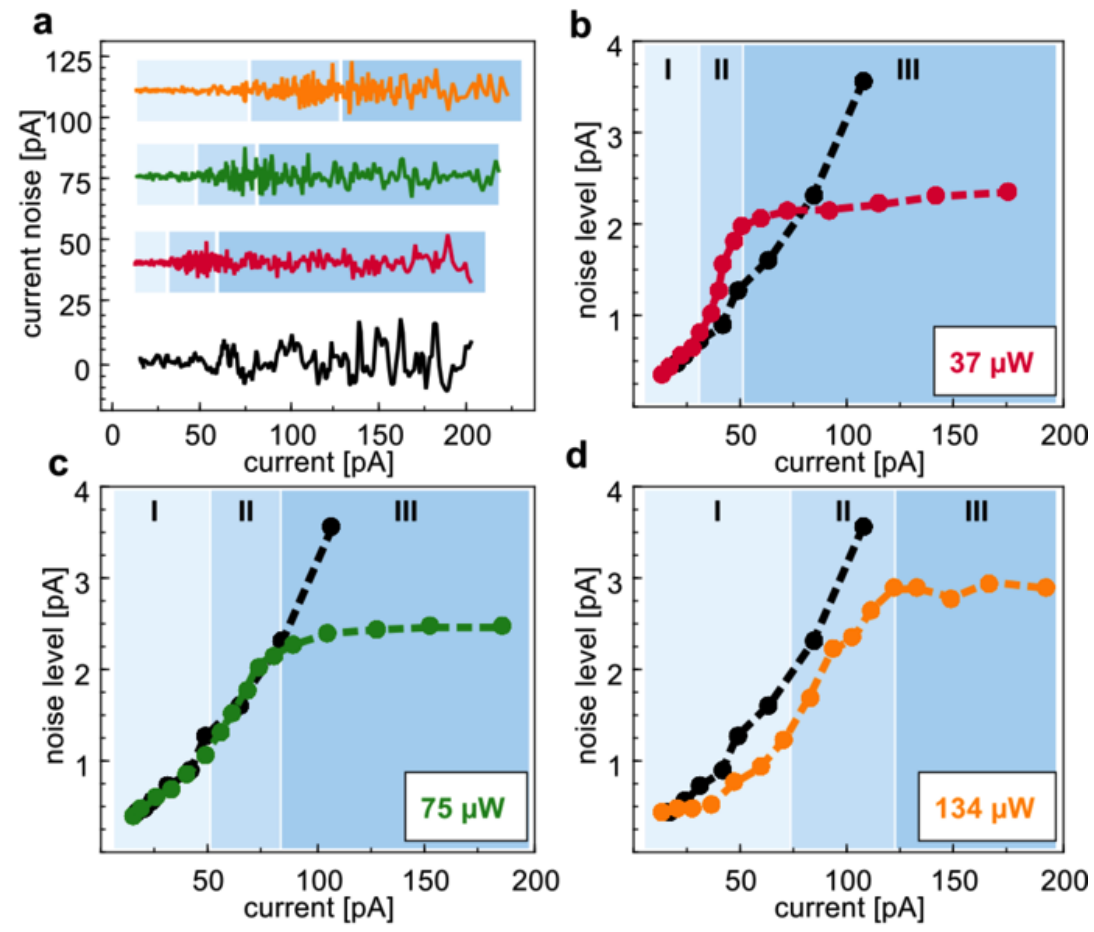

Fig. 3.7 Noise analysis of the $I(z)$ spectra. (a) Current fluctuations of the $I(z)$ spectra (set point $2.5 \mathrm{~V}$ and $10 \mathrm{pA}$ ) plotted against the tunnel current without (black), $37 \mu \mathrm{W}$ (red), $75 \mu \mathrm{W}$ (green) and $134 \mu \mathrm{W}$ (yellow) optical excitation power. The datasets are displaced vertically. (b-d) Noise level $\sigma_{\text {RMs }}$ plotted against tunnel current.

By analyzing the evolution of the noise level for higher optical power steps at $74 \mu \mathrm{W}$ (Fig. 3.7c) and $134 \mu \mathrm{W}$ (Fig. 3.7d) this free charge stabilization in the bulk material can actually reduce the noise induced by space charge dynamics even in I and II. In this $z$-range $\sigma_{\mathrm{RMS}}$ decreases monotonically for increasing $\mathrm{P}_{\mathrm{L}}$. In particular for an optical power of $134 \mu \mathrm{W}$ (Fig. 3.7d) the noise level is below the values without optical excitation showing that charge switching of bi-stable dopants is reduced.

From previous studies, the noise level is expected to increase linearly with the tunnel current up to a tunneling regime close to the point contact of the STM tip ${ }^{97}$. In contrast in regime III saturation in $I_{\mathrm{T}}$ of $\sigma_{\mathrm{RMS}}$ can be observed (Fig. 3.7b-d) indicating that the resulting $\sigma_{\mathrm{RMS}}$ is defined by processes independent of the tunnel current for the optical excited surface. Regarding the dependency on the optical excitation power we observe a slight increase of $\sigma_{\text {RMS }}$ with increasing $P_{\mathrm{L}}$. Nevertheless the saturation behaviour for increasing tunnel currents implies that in the frequency range up to $1 \mathrm{kHz}$ charge fluctuations are the dominant contributions to the noise characteristic. 


\section{Discussion}

In summary, our results show that tunneling into minority charge carriers at the surface allows to actively tune the screening process and thereby to decouple the SCR from parameters like the gate voltage or gate geometry. By controlling the density of minority charge carriers we are able to suppress the noise in this field determined, nanoscopic system considerably. For a more detailed analysis of the dynamics a resolution beyond the $\mathrm{kHz}$ bandwidth of the STM is required. This can be achieved using pulsed optical-excitation ${ }^{25,54}$, which gives the possibility to gain deeper insight into the time scales of the interplay of the tunnel current $\mathrm{I}_{\mathrm{T}}$ and the minority carrier current $I_{\mathrm{H}}$.

\section{Methods}

The experiments are performed in a custom-built, low temperature STM working under UHV condition ( $6 \mathrm{~K}$ at a base pressure of $p<3 \mathrm{e}^{-11} \mathrm{mbar}$ ). For optical excitation, a stabilized continuous wave diode laser at a wavelength of $785 \mathrm{~nm}$ and a maximum power of $100 \mathrm{~mW}$ is used. The focus diameter of the laser is about $50 \mu \mathrm{m}$. With an illumination angle of roughly 30 degrees this results in an optical excited area of $1.57 \cdot 10^{-8} \mathrm{~m}^{2}$. The tips are electrochemically etched from a polycrystalline tungsten wire. The typical tip radius is about $10-50 \mathrm{~nm} . I(V, z)$ spectroscopy is used to study the effect of the tunneling process on the photo-generated charge.

\section{Supplemental Material}

\section{S3.1: Addressing the valence and conduction band surface states}

In the main text it is stated that the topographies show the atomic corrugation for valence band states and conduction band states at positive bias voltages. Ebert et al. ${ }^{33}$ have shown that it is possible to address three different surface states revealing different atomic corrugations in STM topographies. For conduction band tunneling one has the transition from a chess-board like pattern for higher voltages (Fig. 3.8 at $1.2 \mathrm{~V}$ ) to a row-like symmetry by decreasing the bias voltage (Fig. 3.8 at $0.7 \mathrm{~V}$ ). For valence band tunneling at negative bias voltages the atomic pattern shows also a row-like pattern but rotated by 90 degrees. We observe this pattern at low positive bias voltages proofing the possibility to address the photo-generated minority charge carriers (Fig. 3.8 at $0.3 \mathrm{~V}$ ). 


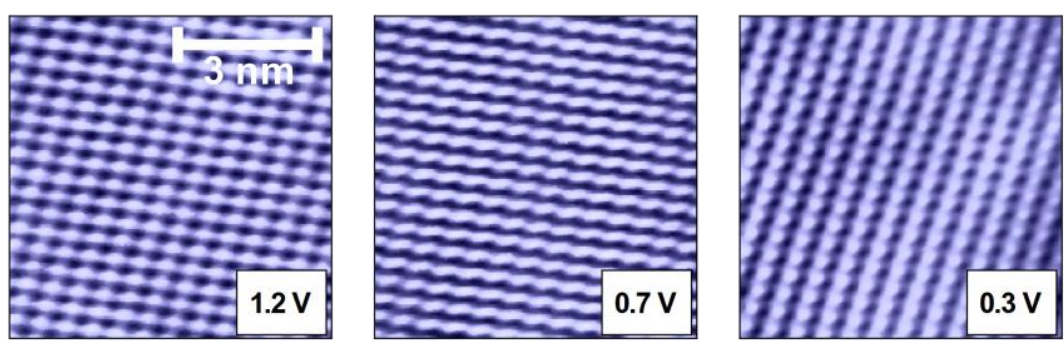

Fig. 3.8: Constant current topographies at the optical excited GaAs(110). For higher voltages (0.7 $\vee$ and $1.2 \mathrm{~V})$ the visible corrugation are characteristic for conduction band tunneling. At $0.3 \mathrm{~V}$ one observes the atomic pattern for valence band tunneling.

In order to ensure the comparability of the $I(z)$ curves for different optical power ratings we have analyzed how the tip-sample distance at the set point is influenced by the excitation. This can be best estimated in regime I, i.e. for low tunnel currents. All $I(V, z)$ datasets are recorded at a set point of $2.5 \mathrm{~V}$ and $10 \mathrm{pA}$. The topographies in Fig. 3.8 show that for high bias voltages the tunnel current mainly consists of conduction band tunneling. The model proposes flat band conditions at low currents in regime I. The optical induced additional valence band tunneling at the set point is defined by the hole density needed to generate these flat band conditions. This density is independent of the applied optical excitation. Therefore we do not expect a change in the net tunnel current $I_{\mathrm{T}}$ when changing the excitation power and therefore identical tip sample distances for each dataset. This allows us to directly compare the recorded $I(z)$ spectroscopies. Fig. 3.9 shows $I(z)$ curves at different excitation densities. In regime I the curves coincide in one master curve.

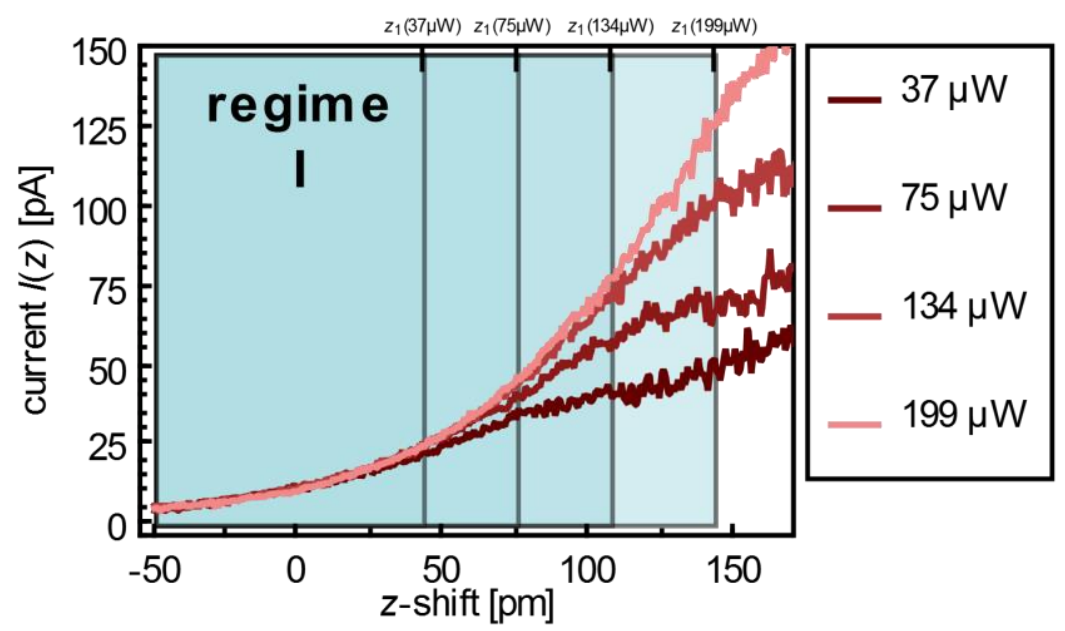

Fig. 3.9 I(z) characteristics at different optical power rates. In the $z$-range of regime I the curves coincide in one master curve ( $2.5 \mathrm{~V}$ and $10 \mathrm{pA})$. The blue shadings mark the $z$-range of regime I for each excitation power.

\section{S3.2: Conduction band tunneling versus Valence band tunneling}

In the topographic analysis we observe dominant valence band tunneling at a bias voltage of $0.3 \mathrm{~V}$. Nevertheless also a current into the conduction band is expected. The determination of the 
dominant tunneling channel is a dedicated task. Qualitatively we can estimate this by comparing the density of states and the transmission probability for valence and conduction band tunneling. Ref. $^{34}$ shows the Local Density of States (LDOS) for the GaAs(110) surface energetically resolved. For negative energies for the valence band the LDOS is nearly twice as high in comparison the LDOS for the conduction band. In order to estimate the transmission probability we use an $I(V)$ spectra from Ref. ${ }^{88}$. At $0.3 \mathrm{~V}$ bias voltage we have only small tunnel currents. Comparing the amount with current for negative voltages (the energetic position of the photo-generated holes) we expect at least the same or even more current. This rationalizes the observation of mainly valence band tunneling at $0.3 \mathrm{~V}$ but also the change of the dominant tunnel channel by going to higher voltages.

\section{S3.3: Extraction of apparent barrier height in $I(z)$ spectroscopy}

In standard tunnel theory the dependency of the tunnel current on the tip sample distance $\mathrm{z}$ is described as:

$$
I(z) \propto \exp (2 \alpha z)
$$

$\alpha$ describes the change in tunnel current when changing $z$ and consists among other values of the apparent barrier height $\Phi$ of the tunnel junction. Therefore by plotting $I(z)$ spectroscopies on a logarithmic scale (Fig. 3.10) the slope of the curves gives directly access to the barrier height $\Phi$.

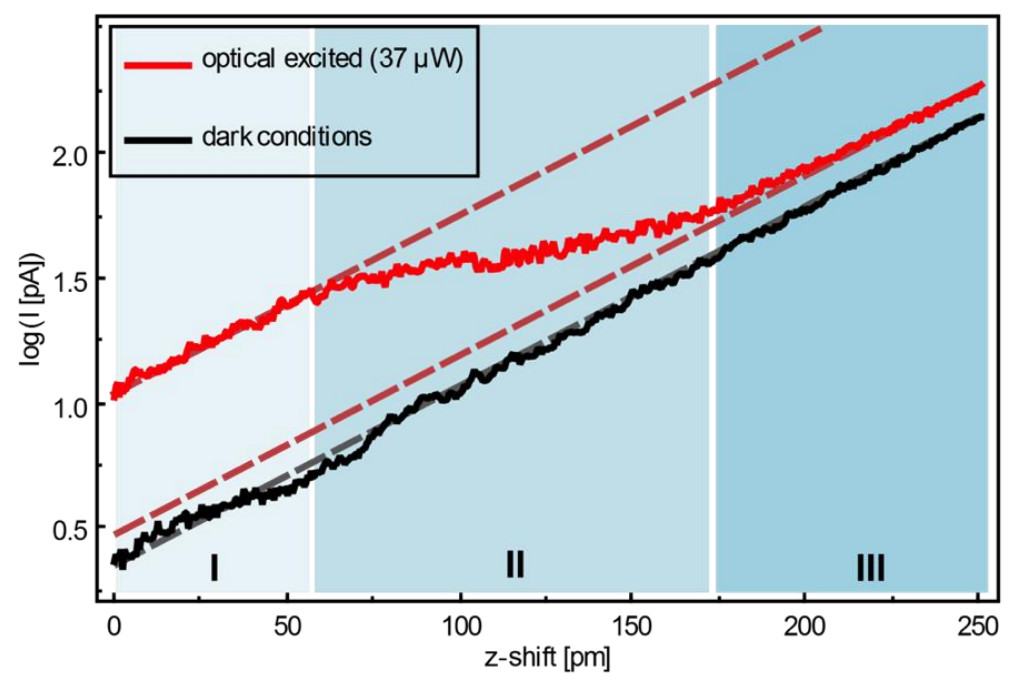

Fig. 3.10 I(z) curves with (red) and without (black) optical excitation plotted on a logarithmic scale. In regime I and II the slope of the curves for the optical excited case is identical to the slope for the data points under dark conditions (set point $2.5 \mathrm{~V}$ and $10 \mathrm{pA})$.

The trend predicted by eqn. 1 can be nicely seen in case of the curve under dark conditions (Fig. 3.10, black curve) showing a clear linear dependency and allows extracting an apparent barrier height $\Phi$ of $2.5 \mathrm{eV}$. This value is characteristic for conduction band tunneling at the GaAs surface. In the optical excited case the run of the $I(z)$ curve is more complicated. Nevertheless after dividing the curve in the three different tunneling regimes (Fig. 3.10, red curve) one observes an identical slope in regime I and III in comparison to the slope of the curve under dark conditions. 
This means that in these regimes the apparent barrier height is the same with and without excitation. Consequently in all cases conduction band tunneling is the predominant process.

\section{S3.4: Modeling and extracting the hole current $I_{\mathrm{H}}$ in regime}

In the following we give a detailed description on how to model and to extract the hole current $I_{\mathrm{H}}$. Without electron injection by the tunnel current, the density of the hole gas at the surface is defined by the potential between tip and sample (Fig. 3.11). Neglecting recombination, there are two currents of minority charge carriers, namely a diffusive component $I_{\text {diff }}$ and a drift component $I_{\text {drift- }}$ Both components add up to a net current, which is zero under equilibrium conditions. Distorting this balance by electron injection via $I_{\mathrm{V}}$, the drift component increases in order to restore the original state. The net current is then the restoring current, which we call the hole field induced (h.f.) current $I_{\mathrm{H}}^{\text {h.F. }}\left(P_{\mathrm{L}}\right)$. In regime I this restoring process is faster than the tunneling rate of $I_{\mathrm{V}}$.

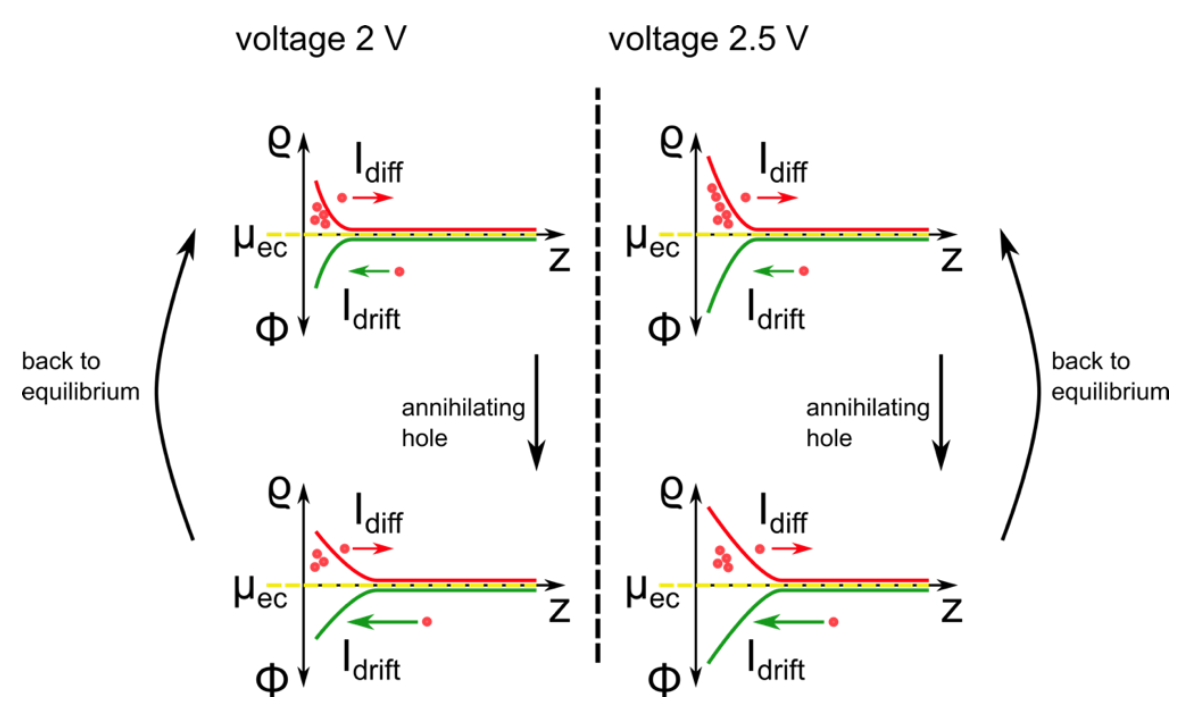

Fig. 3.11 In steady state conditions the hole gas is maintaining balance by a diffusive current $I_{\text {diff }}$ and a drift current $\boldsymbol{I}_{\text {drift }}$ counteracting each other. This equilibrium is given by the density of holes at the surface, which in return is defined by the potential between tip and sample. Electron injection by the tunneling process distorts the system. Consequently the drift component becomes larger than the diffusional one, driving it back into balance. For the low tunneling currents in regime I this process is faster than the electron injection by $I_{V}$. Here the potential between tip and sample is solely screened by the photo-generated holes and we have flat band conditions for all tunnel currents.

At the position of $z_{1}$ we assume that the tunneling into the photo-generated holes $I_{\mathrm{V}}(z)$ has balanced this restoring current towards the surface $I_{\mathrm{H}}^{\text {h.F. }}\left(P_{\mathrm{L}}\right)$. In regime I we describe the tunneling into the valence band states with an exponential dependency on the tip height $z$

$$
I_{V}(z)=I_{V}^{0} \exp \left(2 \alpha_{2} z\right)
$$

with $\alpha_{2}=\sqrt{2 m_{e} \Phi_{V}} / \hbar$ with the apparent barrier height $\Phi_{V}$ for valence band tunneling. Then it yields at $z_{1}$ 


$$
\begin{gathered}
I_{\mathrm{H}}^{\text {h.F. }}\left(P_{L}\right)=I_{V}^{0} \exp \left(2 \alpha_{2} z_{1}\right) \\
z_{1}=\log \frac{I_{\mathrm{H}}^{\text {h.F. }}\left(P_{L}\right)}{I_{V}^{0}} / 2 \alpha_{2} .
\end{gathered}
$$

In order to analyze the $P_{\mathrm{L}}$ dependency of $I_{\mathrm{H}}^{\text {h.F. }}\left(\mathrm{P}_{\mathrm{L}}\right)$ we plot $z_{1}$ against the optical excitation power (Fig. 3.12). In a first approximation $z_{1}\left(P_{\mathrm{L}}\right)$ shows a logarithmic behavior. Taking the exponential dependence of the tunnel current on the distance into account, we describe $I_{\mathrm{H}}^{\text {h.F. }}\left(P_{\mathrm{L}}\right)$ with a linear dependency on $P_{\mathrm{L}}$.

$$
I_{\mathrm{H}}^{\mathrm{h} . \mathrm{F}}\left(P_{L}\right)=m_{\mathrm{h} . \mathrm{F}} \cdot P_{L}
$$

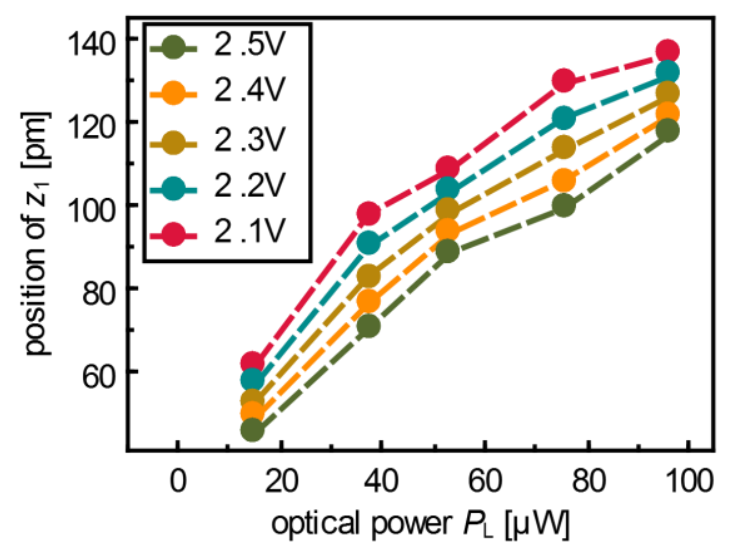

Fig. $3.12 z_{1}$ in the $l(z)$ curves plotted against the applied optical power. In a first approximation the data points follow a logarithmic dependency.

To calculate the capture rate of holes in regime I $\left(m_{\text {h.f. }}\right)$ we use the balance of valence band tunneling and hole diffusion towards the surface at $z_{1}$. The dots in Fig. 3.13 show the current at $z_{1}$ plotted against the laser power $P_{\mathrm{L}}$ for different bias voltages. This current is composed of the tunneling into the valence band modeled by eqn. ( 5 ) and the tunneling into the conduction band modeled by an exponential dependency on the tip height $z$

$$
I_{C}(z)=I_{C}^{0} \exp \left(2 \alpha_{1} z\right)
$$

with $\alpha_{1}=\sqrt{2 m_{e} \Phi_{C}} / \hbar$ defined by the barrier height $\Phi_{C}$ for conduction band tunneling. The overall current at can be written as

$$
I\left(z_{1}\right)=I_{C}^{0} \exp \left(2 \alpha_{1} z_{1}\right)+m_{\text {h.F. }} P_{L} .
$$

With the help of eqn. ( 4 ) we can transform this into

$$
I\left(z_{1}\right)=I_{C}^{0}\left(\frac{m_{\text {h.F. } P_{L}}}{I_{V}^{0}}\right)^{\frac{\alpha_{1}}{\alpha_{2}}}+m_{\text {h.F. }} P_{L}
$$

It yields 


$$
I(z=0)=I_{C}^{0}+I_{V}^{0}
$$

so that we can rewrite $I_{C}^{0}$ and $I_{V}^{0}$ as

$$
I_{C}^{0}=\beta \cdot I(z=0) \text { and } I_{V}^{0}=(1-\beta) \cdot I(z=0)
$$

Eqn. (8) is dependent on the laser power $P_{\mathrm{L}}$ with the unknown parameter $m_{\mathrm{h} . \mathrm{f} \text {. }}$ The ratio of valence and conduction band tunneling at the set point is modeled by $\beta$ in eqn. ( 10 ). For the tunnel barrier $\Phi$, included in $\alpha_{1} / \alpha_{2}$, the extracted result of $2.5 \mathrm{eV} / 4 \mathrm{eV}$ were used (the barrier height for valence band tunneling is complemented by the additional barrier induced by the band gap of GaAs).

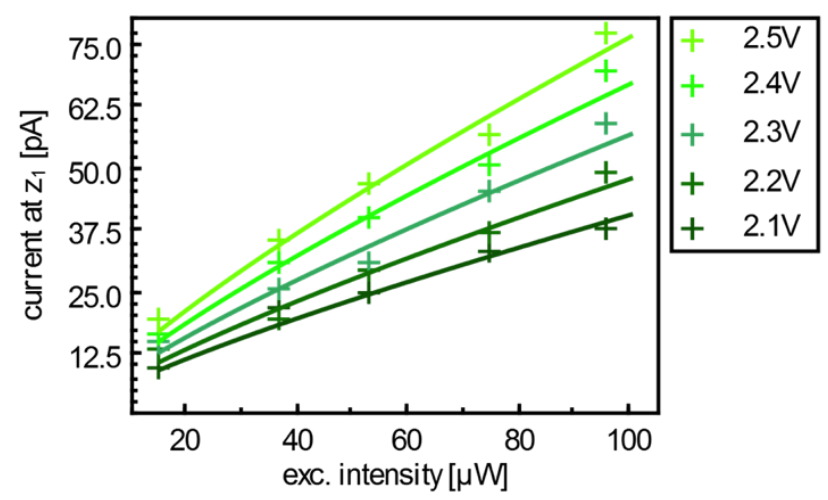

Fig. 3.13 Tunnel current at $z_{1}$ plotted against the applied optical excitation power (circles) for different bias voltages. The solid lines show the result of the fitting to eqn. 8 .

We use a nonlinear fitting routine to adjust eqn. ( 8 ) to the experimental tunnel current at $z_{1}$. As a side condition of the routine, the parameter $\beta$ was set to increase monotonically with the bias voltage, as we expect more tunneling into the conduction band for higher voltages.
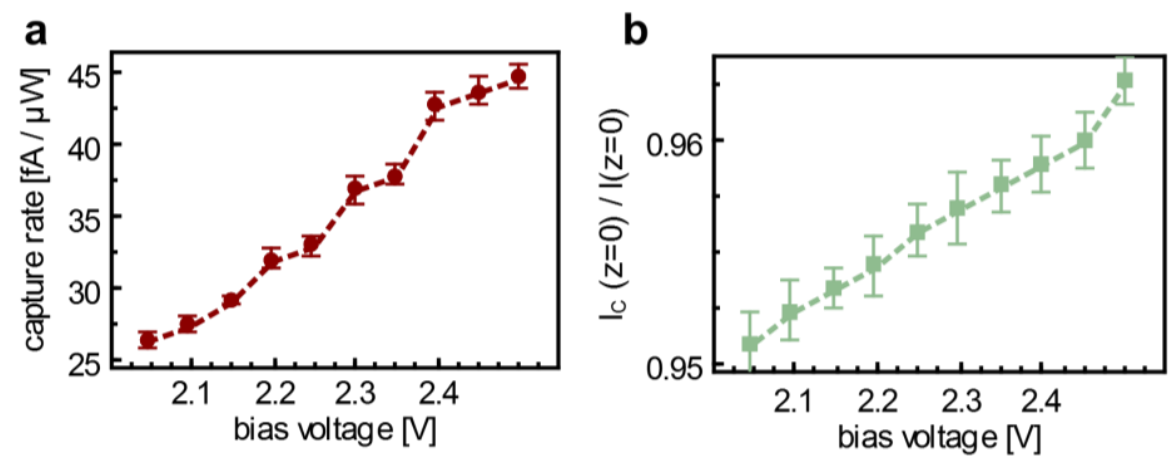

Fig. 3.14 (a) Diffusive capture rate of holes $m_{\text {h.F. }}$ plotted against bias voltage. (b) Ratio of conduction band tunneling in relation to the overall tunneling $\beta$ at the set point $(z=0)$.

The result of the fitting routine can be seen in the solid lines (Fig. 3.13). Fig. 3.14 shows the adjusted fitting parameters $m_{\text {h.F. }}$ (a) and $\beta$ (b).

\section{S3.5: Modeling and extracting the hole current $I_{\mathrm{H}}$ in regime III}

In regime III all holes at the surface are instantly annihilated. The SCR and thereby the electric potential at the surface is built up by charged donors (Fig. 3.15). In this highly non-equilibrium 
conditions the diffusive current $I_{\text {diff }}$ towards the bulk material can be neglected. As a result the net current of holes $I_{\mathrm{H}}^{\text {D.F. }}\left(P_{\mathrm{L}}\right)$ is dopant field induced (D.F.) and is solely defined by the field induced drift current $I_{\text {drift }}$.

voltage $2 \mathrm{~V}$

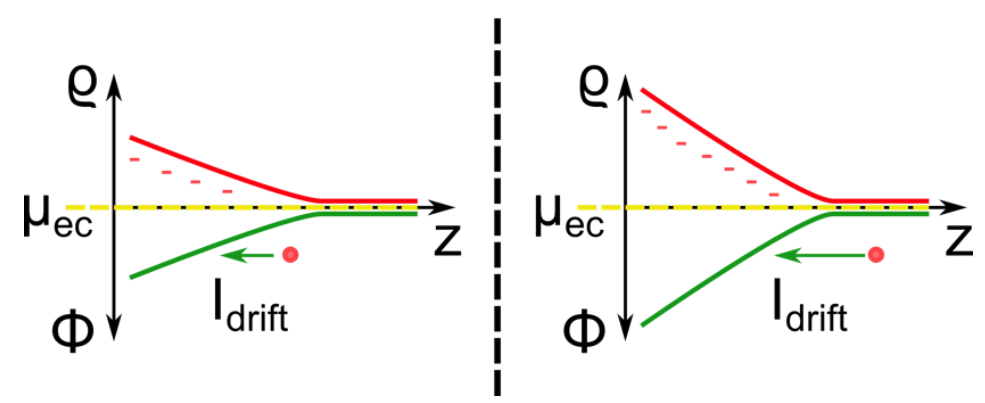

Fig. 3.15 For high tunneling currents the hole gas at the surface is non-existent. The SCR and the electric potential are solely defined by ionized dopants. We expect only a field driven drift current of holes towards the surface $I_{\text {dritt }}$ which is given by the potential between tip and sample.

We assume that the valence band tunneling $I_{\mathrm{V}}(z)$ has overcome this field driven current $I_{\mathrm{H}}^{\mathrm{field}}\left(P_{\mathrm{L}}\right)$. We model the valence band tunneling as a $z$-independent term

$$
I_{V}\left(z>z_{2}\right)=I_{\mathrm{H}}^{\text {D.F. }}\left(P_{L}\right) .
$$

Then we can write the overall current in III as:

$$
I\left(z>z_{2}\right)=I_{C}^{0} \exp \left(2 \alpha_{1} z\right) \cdot \operatorname{TIBB}\left(z_{2}\right)+I_{\mathrm{H}}^{\text {D.F. }}\left(P_{L}\right)
$$

$\operatorname{TIBB}(z)$ describes the current decrease of conduction band tunneling because of the SCR restoring. We compare $I(V)$ spectra with different excitation densities taken in the $z$-range of III (see Fig. 3.16a). The identical curvature of the $I(V)$ data points show that these curves only differ in an offset, i.e. shift along the y-axis. As this shift corresponds to the laser induced current into the valence band $I_{\mathrm{V}}$, we can extract $I_{\mathrm{H}}^{\mathrm{D} . F .}$ by plotting the tunnel current $I_{\mathrm{T}}$ against the corresponding excitation power $P_{\mathrm{L}}$ (see circles in Fig. 3.16b) for a given bias voltage.

$$
I\left(z>z_{2}\right)=I_{C}^{0} \exp \left(2 \alpha_{1} z\right) \cdot \operatorname{TIBB}\left(z_{2}\right)+I_{\mathrm{H}}^{\mathrm{D} . \mathrm{F}} \cdot\left(P_{L}\right)
$$


a

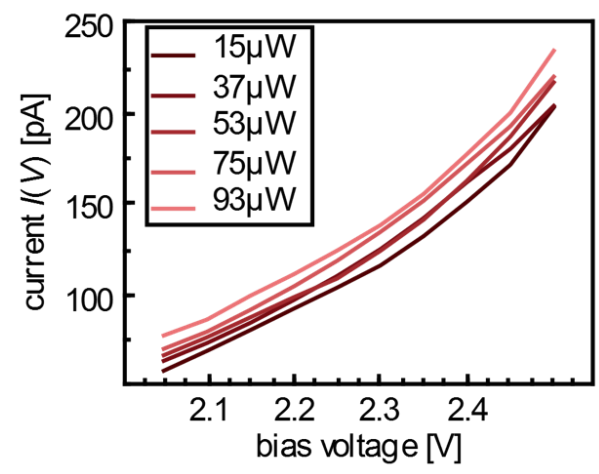

b

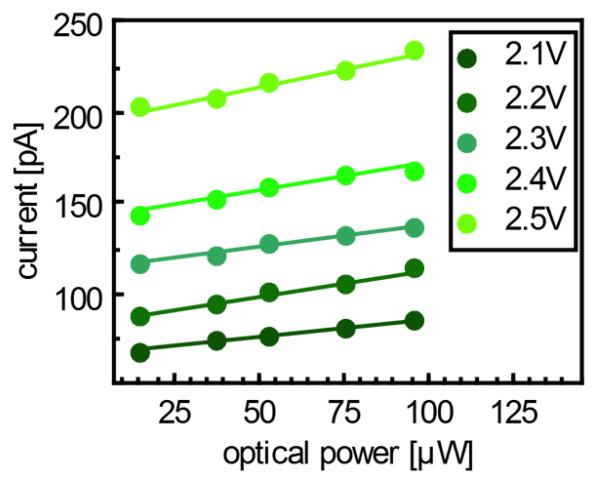

Fig. 3.16 (a) $/(V$ spectra for different optical excitation power ratings in region III. (b) Vertical sections of the $I(V)$ curves shown for different bias voltages. The circles are experimental values. The solid lines a linear fit to these values.

$I_{\mathrm{T}}$ follows a linear dependency on the optical excitation power $P_{\mathrm{L}}$. Therefore we model the current of holes towards the surface in this region as the dopant field driven capture rate $m_{\text {D.F. }}$ times the laser power $P_{\mathrm{L}}$

$$
I_{V}\left(z>z_{2}\right)=m_{\text {D.F. }} \cdot P_{L}
$$

Linear fitting of the data points in Fig. 3.16b we extract the field driven rate $m_{\text {D.F. }}$ shown in Fig. 3.17.

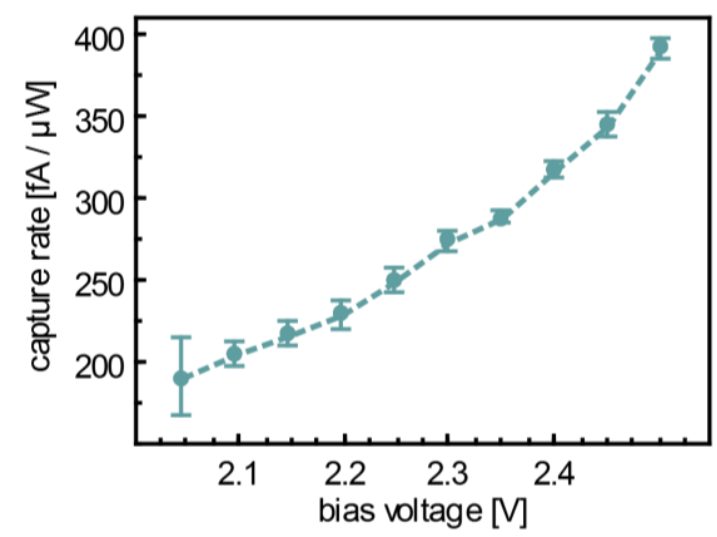

Fig. 3.17 Dopant field

driven capture rate $m_{\text {D.F. }}$.

plotted against bias

voltage.

\section{S3.6: Adjusting the model quantitatively to the experimental $I(z)$ curves}

This chapter describes the quantitative modelling of the experimental data. In the main article we proposed to describe the recorded $I(z)$ characteristics as the sum of valence band $I_{\mathrm{V}}(z)$ and conduction band tunnelling $I_{\mathrm{C}}(z)$. Then, according to the model for both channels for a given bias voltage, it yields: 


$$
\begin{gathered}
I_{V}(z)=\left\{\begin{aligned}
I_{V}^{0} \exp \left(2 \alpha_{2} z\right), & \text { if } z<z_{1} \\
m_{\mathrm{h} . \mathrm{F} .} \cdot P_{L}, & \text { if } z=z_{1} \\
m_{\text {D.F. }}(z) \cdot P_{L}, & \text { if } z_{1}<z<z_{2} \\
m_{\text {D.F. }}\left(z_{2}\right) \cdot P_{L}, & \text { if } z>z_{2}
\end{aligned}\right. \\
I_{C}(z)=\left\{\begin{array}{cl}
I_{C}^{0} \exp \left(2 \alpha_{1} z\right), & \text { if } z<z_{1} \\
I_{C}^{0} \exp \left(2 \alpha_{1} z\right) \cdot T I B B(z), & \text { if } z_{1}<z<z_{2} \\
I_{C}^{0} \exp \left(2 \alpha_{1} z\right) \cdot T I B B\left(z_{2}\right), & \text { if } z>z_{2}
\end{array}\right. \\
I(z)=I_{V}(z)+I_{C}(z)
\end{gathered}
$$

$\alpha_{1} / \alpha_{2}$ are given by the barrier height of the tunneling electrons $\Phi_{1} / \Phi_{2}$ determined to $2.5 \mathrm{eV} / 4 \mathrm{eV}$ respectively. The position of $z_{1} / z_{2}$ in the $I(z)$ curves can be directly extracted from the experimental data. The calculation of $m_{\text {h.F. }}, m_{\text {D.F. }}\left(z_{2}\right), I_{\mathrm{V}}^{0}$ and $I_{\mathrm{C}}^{0}$ is explained previously in the supplemental material.

The parameters $\operatorname{TIBB}(z)$ and $m_{\text {D.F. }}(z)$ are yet undetermined and are strongly related to the restoring process of the SCR at the surface in region II. The model value $\operatorname{TIBB}(z)$ describes the current decrease of the conduction band tunnelling by going from flat band conditions to the tip induced field found at the surface under dark conditions. The additional capture rate introduced by the built up of the SCR is defined by $m_{\text {D.F. }}(z)$. Whereas the limits of $m_{\text {D.F. }}(z)$ are experimentally defined by $m_{\text {h.F. }}\left(z_{1}\right)$ and $m_{\text {D.F. }}\left(z_{2}\right)$, the maximum current decrease $\operatorname{TIBB}(z)$ for the fully restored SCR is adjusted to the experimental data.

We assume that the restoring of the SCR is linearly connected to the applied tunnel current $I(z)$. Then we describe the run of the model parameters $\operatorname{TIBB}(z)$ and $m_{\text {D.F. }}(z)$ with an exponential dependency on the tip height $z$ in the z-range between $z_{1}$ and $z_{2}$.

$$
\begin{gathered}
\operatorname{TIBB}(z)=\Lambda+(1-\Lambda) \cdot \exp \left(-\frac{1}{\lambda} \cdot\left(z-z_{1}\right)\right) \\
m_{\text {D.F. }}(z)=m_{\text {h.F. }}\left(z_{1}\right)+m_{\text {D.F. }}\left(z_{2}\right) \cdot\left(1-\exp \left(-\frac{1}{\lambda} \cdot\left(z-z_{1}\right)\right)\right) .
\end{gathered}
$$

$\Lambda$ defines the percentage of conduction band tunnelling due to TIBB restoring which remains at $z_{2}$. $\lambda$ is given by a fraction of the size in $z$ of regime II. A quantitative comparison of this model with experimental data is shown Fig. 3.18. 
a

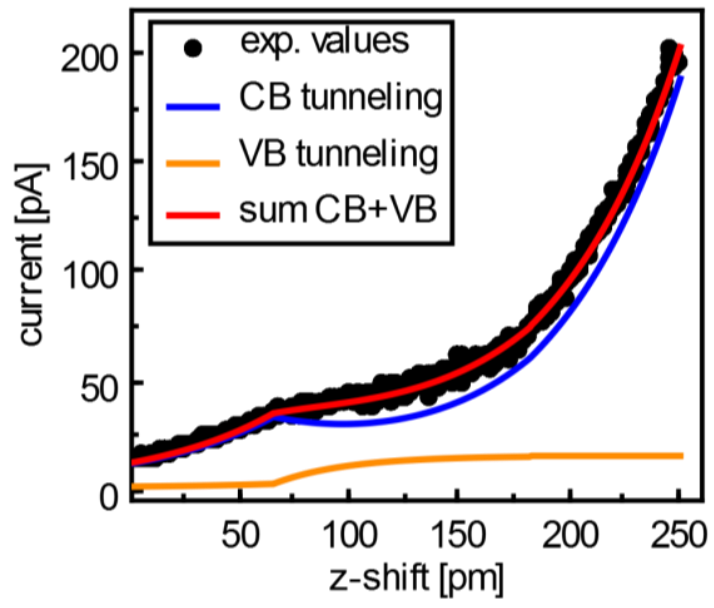

b

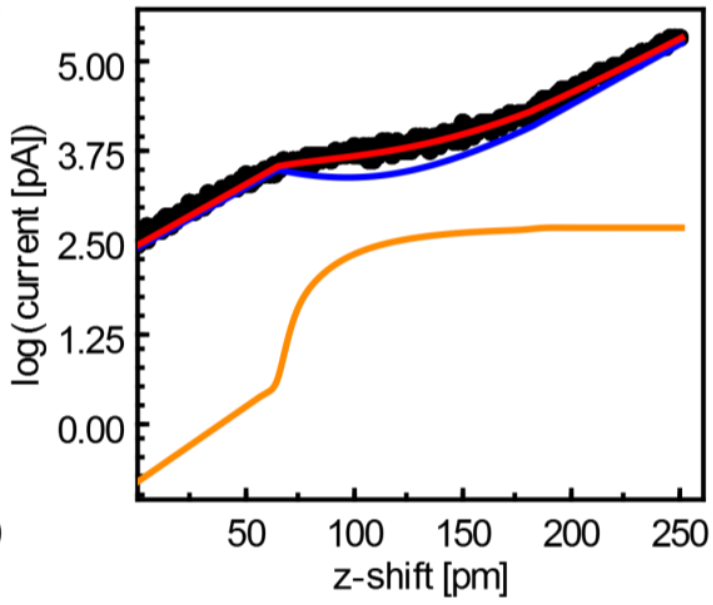

Fig. 3.18 (a) Comparison of model and experimental data for an $I(z)$ spectra taken with $37 \mu \mathrm{W}$ optical excitation and a set point of $2.5 \mathrm{~V}$ and $10 \mathrm{pA}$. (b) Identical dataset but here plotted on a logarithmic scale. Adjusted parameters: $\Lambda=0.2$ and $\lambda=35 \mathrm{pm}$.

\subsection{General discussion}

Measuring the SPV with an STM has been already done extensively ${ }^{11-13,15,60,79}$. However, the influence of the tunnel current has been widely neglected or has been only treated superficially ${ }^{13,17,79}$. In contrast to previous studies ${ }^{13,17}$, the imaging of valence band states at positive bias voltages is the first direct proof, that photo-generated charge must be included in the tunneling process. By performing $I(z)$ spectroscopies, different regimes of hole concentrations at the surface can be accessed. These regimes are directly connected to the excitation intensity as well as the tip induced potential. A simple model including the additional tunnel channel into the photo-generated charge allows extracting intrinsic values like the diffusion or drift rate of holes towards the surface.

By using the tunnel current as an additional control parameter, the study of SPV with an STM evolves to a new complexity. Up to now, the determination of photo-induced potential changes at the surface has been resolved by comparing the shift in $I(V)$ spectra with and without laser illumination (see Fig. 3.1). With the presented results of chapter 3.3 it is clear, that this is only half of the truth. The additional tunnel channel into valence band states is always masked in the $I(V)$ spectra. In order to extract the change of the surface potential, one has to disentangle the induced shift in the curves by pure "photo-potential" effects from the counter-acting process of charge annihilation via the tunnel current. Otherwise, this extra contribution could be interpreted as a SPV by mistake. In an extreme case, for high tunnel currents, no photovoltage is present. However, the additional tunneling into photo-generated charge will result in a shift of $I(V)$ curves, which is eventually everything else than an SPV.

Beyond SPV-STM experiments, the influence of currents on the surface potential has already been treated. For example photoemission spectroscopy has been used at surfaces to determine values like 
the Schottky barrier. In this approach the congruent to the tunnel current is a leakage current along the surface, annihilating photo-generated charge. Chen et al. ${ }^{93}$ investigated this effect by the controlled deposition of Platinum layers on GaP. Thereby, it was possible to vary the leak current by varying the thickness of the metal ad-layer and with this the potential at the surface.

The tuning of the hole density via the tunnel current of an STM is a way more controlled method. In this context, the additional tunnel channel bears new possibilities, e.g. studying different recombination rates inside the tip-induced, nanoscaled space charge region.

a

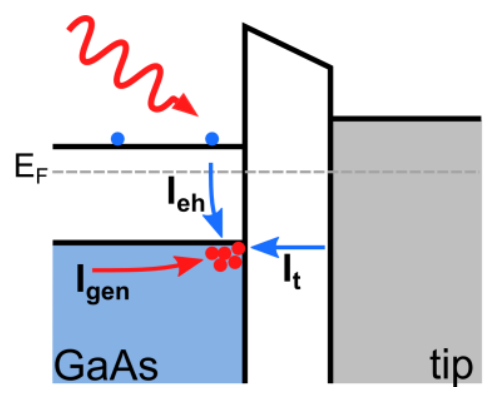

b

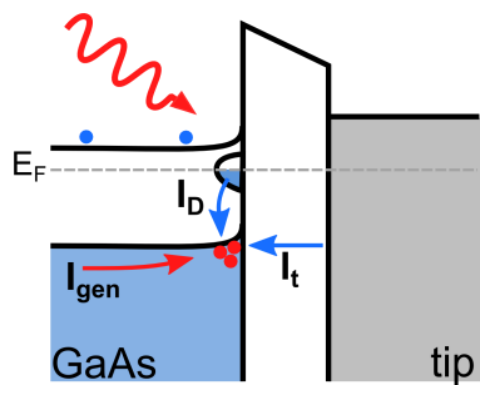

Fig. 3.19 Steady states of charge generation and charge annihilation at the free GaAs surface (a) and in the presence of a deep level defect state (b) at the GaAs surface.

In general, the density of photo-generated charge at defect-free surfaces is determined by a steady state between generation and recombination (Fig. 3.19a). In the previous section (chapter 3.3) it was shown, that the hole generation current towards the surface $I_{\text {gen }}$ is determined by the tipinduced potential and the optical excitation density. Recombination processes are given by electron-hole recombination, summarized as the current $I_{\text {eh }}$, or charge annihilation via valence band tunneling $I_{\mathrm{t}}$. The defect-free $\operatorname{GaAs}(110)$ has a low surface carrier recombination rate ${ }^{11,46}$, i.e. $I_{\text {eh }}$ is negligible. At local defects, characterized by deep level states inside the band gap, an extra recombination mechanism $I_{\mathrm{D}}$ at the Fermi energy comes into play (Fig. 3.19b). The total number of charge carriers at the surface adjusts, corresponding to a new steady state ${ }^{\S}$. In the $I(z)$ spectra we cant extract this equilibrium when for all rates yield $I_{\text {gen }}=I_{\mathrm{D}}+I_{\mathrm{t}}$. Subsequently, by tuning the tunnel rate $I_{\mathrm{t}}$ and optical excitation intensities $I_{\text {gen }}$, the defect related recombination rate $I_{\mathrm{D}}$ can be calculated. Moreover, the spatial influence of this particular defect can be extracted by acquiring $I(z)$ spectra point by point at different positions in its neighborhood.

At last, the observation of changing noise levels of the tunnel current in the three tunneling regimes with optical excitation is discussed. In order to elucidate these phenomena and disentangle involved mechanisms, a time-resolved study is needed. The next chapter is a first step into this direction.

\footnotetext{
${ }^{\S}$ It is assumed that $I_{\mathrm{D}} \gg I_{\text {eh }}$. Therefore, in Fig. 3.19b the e-h recombination is omitted for simplification.
} 



\section{$4 \quad$ Pulsed optical excitation at the GaAs(110) surface}

In order to demonstrate the powerful technique of pulsed optical excitation in STM experiments (SPPX-STM) a suited sample system is necessary. Materials like semiconductor surfaces are an obvious choice. Due to the rather easy preparation method in UHV (see chapter 1.1) and also due to its well-known sample properties, GaAs is a promising prototype candidate in not only acting as a proof of concept of the SPPX-STM but also by providing insights into complex charge carrier dynamics (a summary of microscopic processes is given in the chapter 1.2 and Fig. 1.7). Usually, these dynamics have only been resolved on a macroscopic scale by photo-luminescence spectroscopy ${ }^{98}$, Surface Photovoltage spectroscopy ${ }^{99}$ or electron beam induced current ${ }^{100}$.

The first part of this chapter summarizes previous studies on optical induced charge dynamics investigated via SPPX-STM at the GaAs(110) surface**. Eventually, in chapter 4.4 our own measurements on the charge dynamics at this surface can be found. In contrast to previous results, the focus of these studies lies on the detailed investigation of the tunnel current dependency of the charge dynamics in the tip-induced space charge region. This has been already discussed under continuous excitation in chapter 3. By driving the system in unusual non-equilibrium conditions in a controlled manner, one gets access to the time characteristics of the hole annihilation process but as well to the ionization dynamics of single dopants.

\subsection{The beginnings ${ }^{\dagger \dagger}$}

In 2004, SPPX measurements in combination with STM were made by Shigekawa et al. ${ }^{24}$. First results were obtained using a periodic modulation of the delay time $t_{\mathrm{d}}$ between pump and probe pulse induced by a movable mirror in the delay line of the probe pulse (in detail described in chapter 2.1 and Fig. 2.6). Fig. 4.1 shows the optical induced current $d I\left(t_{\mathrm{d}}\right)$ in the picosecond time regime at the GaNAs surface. The low frequency modulation of $t_{\mathrm{d}}$ via the mirror-movement does not give directly access to the decay of the photo-induced current but instead provides the derivative of it (Fig. 4.1a). After numerical integration (Fig. 4.1b) the decay gets visible as a superposition of two processes with decay constants of roughly 1 and $50 \mathrm{ps}$.

To this day, the physical background of this observation remained unclear. Generally it is found that the time scales are similar to the results of pure optical pump-probe-reflectivity measurements.

\footnotetext{
** Up to now, solely provided by the research group of Hidemi Shigekawa at the University of Tsukuba (http://dora.ims.tsukuba.ac.jp)

$\dagger$ This chapter is dedicated exclusively to the subject pulsed optical excitation at the GaAs surface. Other time-resolved experiments on the atomic scale are shown in chapter 2.1 .
} 
This optical signal is at least two magnitudes higher than the modulation in tunnel current and therefore Shigekawa et al. exclude optical bleaching. In the end, thermal cooling of hot electrons is made responsible for the $t_{\mathrm{d}}$ dependent change in the tunnel current, acting on time scales from single to tenth of picoseconds. However, a crosscheck of this model is missing.
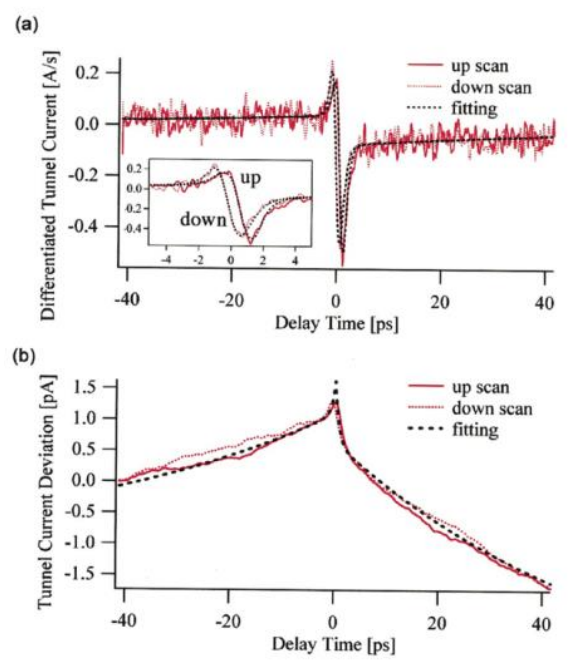

Fig. 4.1 First results of the SPPX induced tunnel current at the GaNAs surface. (a) By modulating the delay time between pump and probe pulse via a mirror movement in the delay line of the probe pulse gives the differentiated tunnel current, plotted against the delay time. (b) Numerical integration gives access to two decaying processes. (-2V, $100 \mathrm{pA}$, exc. parameters: pulse width $25 \mathrm{fs}$, repetition rate $80 \mathrm{MHz}$, average intensity $<10 \mathrm{~mW}$ ). Taken from $^{24}$.

\subsection{Evolution of the SPPX method}

The spectra in Fig. 4.1 show a fast decay of the signal in the picosecond time regime. Additionally, in the background one identifies a much slower decaying process, which could not be resolved in this time frame. To overcome this technical limitation, Shigekawa et al. refined the SPPX method by going to an advanced pulse picking technique ${ }^{52}$, allowing to resolve transients from picoseconds to microseconds (in detail described in chapter 2.1 and Fig. 2.8). For this refined method no subsequent integration is needed.

Fig. 4.2 $d I\left(t_{\mathrm{d}}\right) \quad$ spectra on different semiconductor surfaces shows the large time frame now accessible via the refined SPPX method. (a) LT-GaAs with $\tau_{s}=2.4 \mathrm{ps,} \mathrm{(b)} \mathrm{GaNAs}$ with $\tau_{s}=440 \mathrm{ps}$, (c) high quality GaAs with $\tau_{s}=2.4 \mathrm{~ns}$ and (d) $\mathrm{H}$-Silicon with $\tau_{s}=0.87 \mu \mathrm{s}$. (tunneling parameters are not provided, exc. parameters: pulse width $140 \mathrm{fs}$, repetition rate $1 \mathrm{MHz}$, average photo intensity up to several $\mathrm{mW}$ ). Taken from ${ }^{52}$.

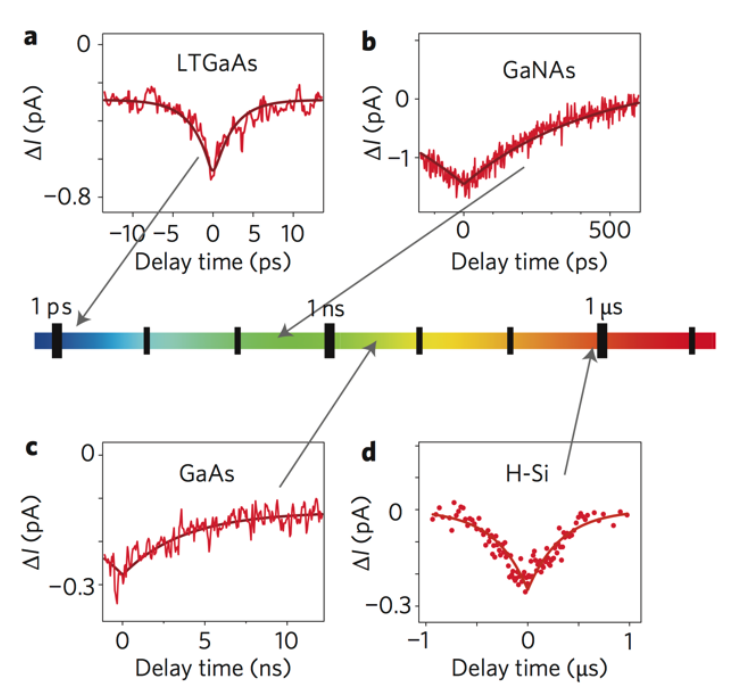


As a proof of principle of their new setup, $d I\left(t_{\mathrm{d}}\right)$ spectra are recorded for a variety of sample surfaces $^{52}$. By going from defect-rich low-temperature grown GaAs (LT-GaAs) (Fig. 4.2a) or GaNAs (Fig. 4.2b) to high quality GaAs (Fig. 4.2c) and the hydrogen-terminated silicon surface (Fig. 4.2d), the decay time varies from single picoseconds to microseconds.

In the following, the text focuses on the charge dynamics at the high quality $\operatorname{GaAs}(110)$ surfaces (Fig. 4.2c). A detailed analysis of the time spectra at this surface reveals, that the decay can be modeled as a superposition of two exponential functions with differing decay constants $\tau_{s}$ and $\tau_{b}$ (Fig. 4.3a). Instead of the fast cooling of hot electrons, like in the previous subchapter (chapter 4.1), the rather long decay constants in the nanosecond time regime are explained by the recombination of photo-generated charge across the band gap. In this context, Shigekawa et al. differentiate between two types of charge carriers: (i) electron-hole pairs in the bulk material and (ii) trapped holes inside the tip-induced SCR at the surface.
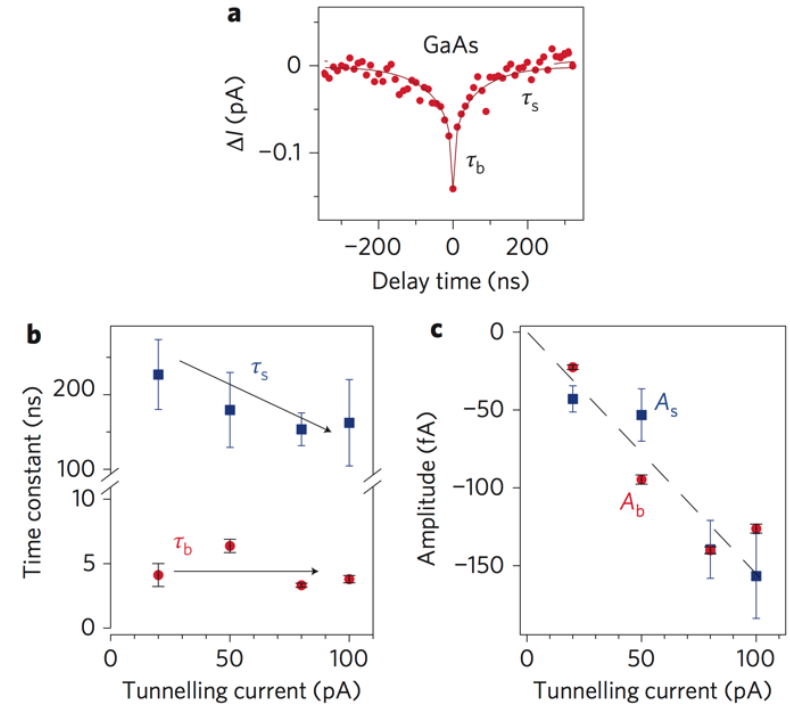

Fig. 4.3 More detailed view on the $d I\left(t_{d}\right)$ spectra at the high quality $\mathrm{GaAs}(110)$ surface. (a) A single $d /\left(t_{d}\right)$ spectra reveals two decaying constants, $\tau_{s}$ and $\tau_{b}$. $(5.5 \mathrm{~V}, 40 \mathrm{pA}$, exc. parameters identical to Fig. 4.2). (b) $A$ current dependent study of the decay constants indicates a dependency of $\tau_{s}$, whereas $\tau_{b}$ remains constant. (c) The absolute value of $d l_{\max }$ increases linearly with the tunnel current. Taken from ${ }^{52}$.

The bulk decay by simple electron-hole recombination is expected to proceed fast and is therefore connected to the decaying signal with the decay constant of roughly $\tau_{b}=5 \mathrm{~ns}$. This value is in convincing accordance to the intrinsic bulk charge carrier decay of GaAs. It becomes more complex for the charge carriers trapped in the surface potential, like already indicated in chapter 1.2 and Fig. 1.7. Shigekawa et al. stated, that tunneling electrons into the conduction band are possible recombination partners for the holes at the surface. Then, consequently, one observes a current dependency on $\tau_{s}$ (Fig. 4.3b).

\subsection{Charge recombination at local surface defects}

For studies on local defects or other, structural inhomogeneities, one has to take defect-induced electronic effects into account. Shigekawa et al. investigated the photo-generated carrier 
recombination in the presence of cobalt clusters (STM topography in Fig. 4.4a) at the GaAs(110) surface $^{52}$.

By taking $d I\left(t_{\mathrm{d}}\right)$ spectra at the defect-free GaAs surface (Fig. 4.4b) and above a cobalt cluster (Fig. 4.4c) a significant change of more than a factor of five in the decay constant $\tau_{s}$ is observed. At the free surface the decay of holes is described as recombination with tunneling electrons. In the presence of the cobalt cluster one has to include the effect of band gap states for the recombination process (Fig. 4.4d). Here, trapped holes at the surface can recombine with electrons of the half filled defect state. In the model of Shigekawa et al., at low voltages ( $2 \mathrm{~V})$ the occupation density can be tuned by changing the tunneling rate into the defect state. This allows distinguishing between two regimes, defining the relaxation rate of the trapped holes at the surface (Fig. 4.4e). At low tunnel currents the relaxation rate of the holes is limited by the electron transfer via tunneling into the defect state, noted as $J_{\mathrm{e}}$. Consequently, in this current range, $\tau_{s}$ is very sensitive to the total amount of tunnel current. In the second regime, for high tunnel currents, $\tau_{s}$ remains constant (Fig. 4.4e). Here, the relaxation rate of trapped holes with filled defect states $J_{\mathrm{h}}$ is limited by the intrinsic properties, e.g. the overlap of the wave functions of the defect states and the holes inside the valence band.
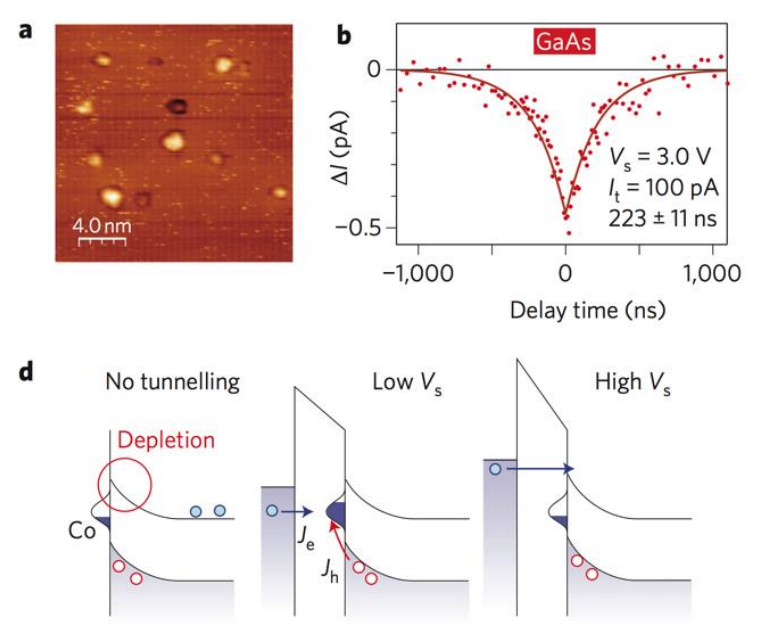
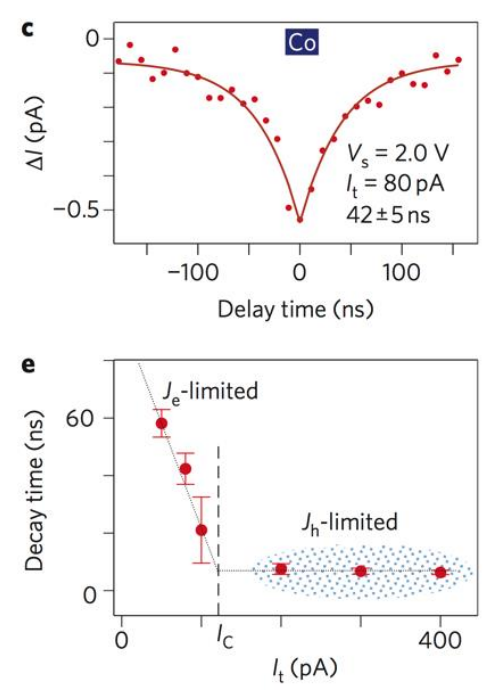

Fig. 4.4 Study of carrier recombination dynamics at cobalt clusters on the GaAs surface. (a) STM topography of cobalt nanoparticles. (b) $d /\left(t_{d}\right)$ spectra on the bare GaAs surface and (c) on a cobalt cluster show significant changes of $\tau_{s}$ above the defect. (d) Model for carrier recombination $J_{\mathrm{h}}$ via defect-induced gap states. In this model tunneling into the defect states $J_{\mathrm{e}}$ is assumed. (e) Depending on the tunneling rate $J_{h}$ different occupation regimes of the defect states can be probed. For high currents the decay of the trapped holes at the surface is limited by the intrinsic rate $J_{h}$. Taken from ${ }^{52}$.

Shigekawa et al. ${ }^{52}$ measured this intrinsic rate $J_{\mathrm{h}}$ laterally and classify it for different cluster sizes. Fig. 4.5a shows an STM topography of cobalt cluster whereas in Fig. 4.5b $\tau_{s}$ is locally resolved for the same area of the surface. As the quality of the $\tau_{s}$-map is quite modest and inhomogeneous, only a superposition with the topography (Fig. 4.5c) allows isolating the signatures at the cobalt clusters 
as a local depression in $\tau$. By extracting $\tau_{s}$ at cobalt cluster of different sizes a clear monotonic decreasing dependency between both values can be determined (Fig. 4.5d).
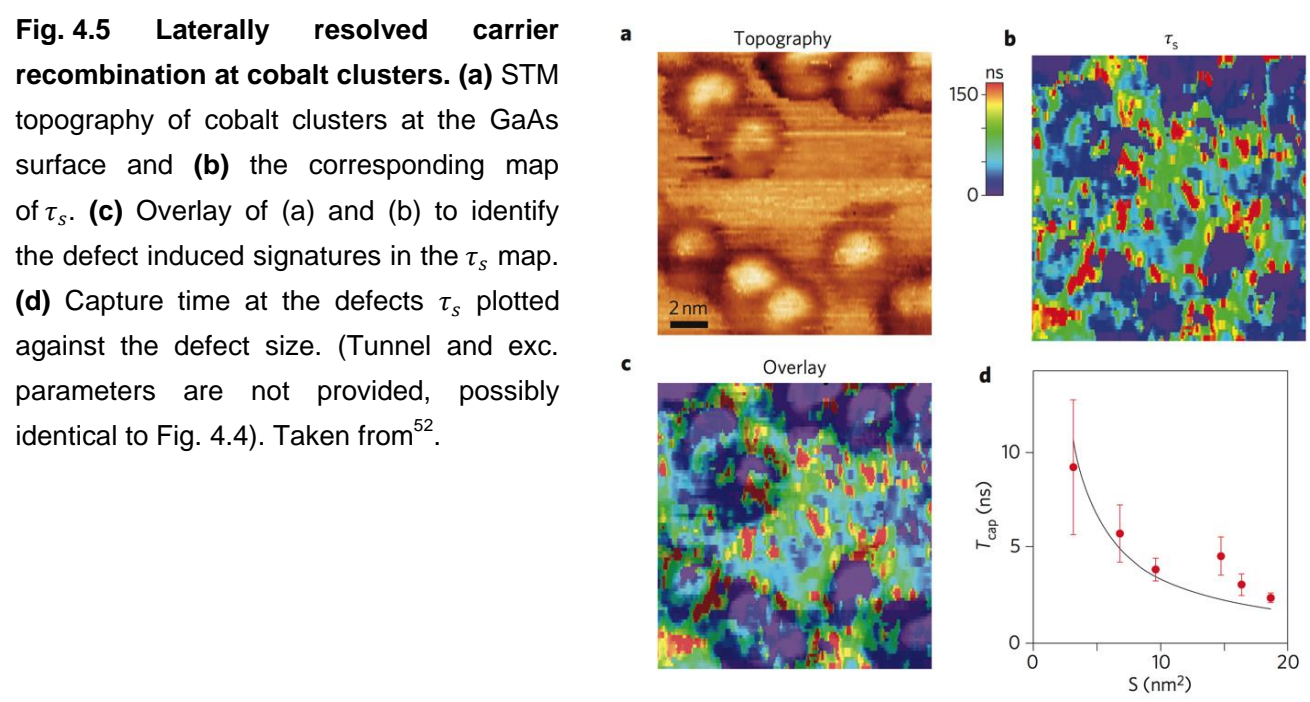

In a next step, Shigekawa et al. deposited single manganese (Mn) atoms on the GaAs(110) surface $^{64}$. Completely analogue to the cobalt cluster, the objective was to investigate the influence of single atomic surface defects on the photo-generated carrier recombination. The STM topography in Fig. 4.6a, b shows the signatures of single Mn atoms on the GaAs surface for positive and negative bias voltage and for high (a) and low (b) laser intensities. At negative bias voltages the topographies are identical, whereas one recognizes an obvious contrast change at positive bias voltages and for low and high excitation intensities. Contrary to the tunnel current dependent study of the different occupation regimes of the defect state, demonstrated in Fig. 4.4e, the difference here of both regimes is shown for varying optical excitation intensities. At positive bias voltages, Shigekawa et al. proposes a combined tunneling into the conduction band and into the defect states directly at the $\mathrm{Mn}$ atoms, resulting in a local enhancement in the topography in (a). In order to make the tunneling into the defect state possible it has to provide empty electron levels. At increased laser intensities, the high concentration of trapped holes and likewise the constant recombination of these holes with electrons in the defect states ensure depopulated band gap levels enabling this additional tunneling channel. By decreasing the laser intensity this process becomes unlikely. The amount of electrons, coming from the tunnel current and occupying the defect state, overcome the recombination rate with trapped holes at the surface. Consequently, this tunnel channel into the defect states freezes out, leading to the local depressions in the topography (Fig. 4.6b). Fig. 4.6c shows cross sections along the local depressions in Fig. 4.6b. As these studies were performed at room temperature, Shigekawa et al. used an STM tip position feedback system at these signatures in order to fix the tip apex above the defect. To extract the intrinsic recombination rate of holes into these defect states, Shigekawa et al. took a single $d I\left(t_{\mathrm{d}}\right)$ spectra (Fig. 4.6d) giving a decay constant of $\tau=14.3 \mathrm{~ns}$. 
(a) high laser intensity

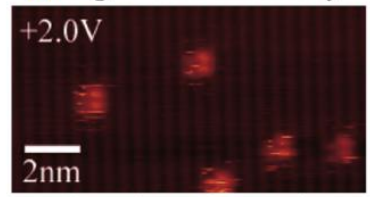

(b) low laser intensity
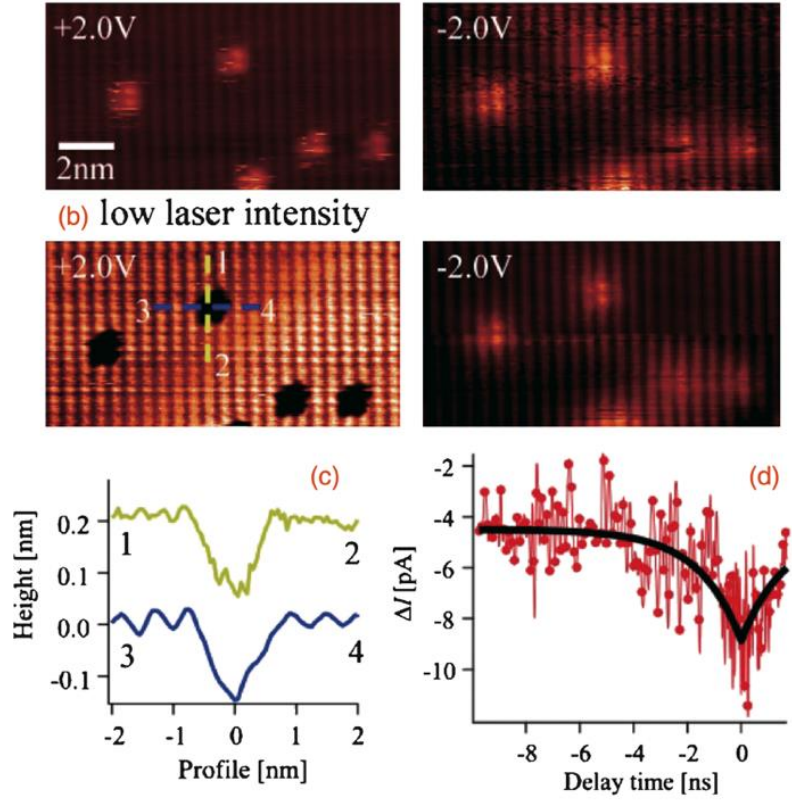

Fig. 4.6 Carrier relaxation study at single manganese $(\mathrm{Mn})$ atoms at the GaAs surface. (a), (b) STM topographies of single $\mathrm{Mn}$ atoms at positive and negative bias voltage and for high $(0.13 \mathrm{~mW})$ and low laser intensity $(0.01 \mathrm{~mW})$. Tunnel current: $100 \mathrm{pA}$. (c) Cross-sections along the local depressions in the topography in (b). (d) Single $d l\left(t_{\mathrm{d}}\right)$ spectrum at the $\mathrm{Mn}$ atom reveals a decay constant of 14.3 ns. (2 V, $100 \mathrm{pA}$, exc. parameters unknown). Taken from ${ }^{64}$.

\subsection{From time-resolved imaging of individual donors to their cooperative dynamics}

After all the effort of the research group of H. Shigekawa, a real atomically resolved analysis of the dynamics at the GaAs surface is still missing. In Fig. 4.5, e.g., a spatiotemporal resolved map of cobalt clusters on this surface is presented. Although, the influence of the cobalt clusters becomes visible in the decay map (Fig. 4.5b), a real evaluation including, e.g., long-range effects of the carrier dynamics, could not be done. For the study of the Mn atoms on the GaAs surface in Fig. 4.6 the spatial resolution of the decay is as well missing.

This subchapter treats the carrier dynamics at the GaAs(110) surface or more general the carrier dynamics (charge transfer, e-h recombination, dopant ionization/neutralization) inside the tipinduced space charge region (SCR) in detail. Previous studies of ours have shown ${ }^{19,87,101}$, that single and combined donor dynamics have massive impact on the SCR, i.e., the tunnel current. This observation triggered the investigation of this electrostatically driven charge dynamics timeresolved and, especially, on the atomic scale. A key-aspect for performing these experiments is the massive reduction of the optical laser intensity. It was shown, that already low optical excitation lead to saturation effects at the GaAs surface ${ }^{11,46}$. But, beyond this saturation, we were able to access different non-equilibrium regimes which allowed us to access and resolve different relaxation mechanisms.

This subchapter is published in identical words in the scientific journal "Science Advances". Again, a brief summary in the beginning and a concluding, more general discussion at the end are given. The manuscript was written in collaboration with Martin Wenderoth. This subchapter 
contains the main manuscript and additional supplementary information (abbreviated with S4.1 S4.9).

\section{Brief summary}

The interplay of optically excited charge carriers with fixed, ionized dopants inside the tip-induced space charge region can result in complex dynamics. In chapter 3.3 it was shown, that the tunnel current is able to drive this specific system in various non-equilibrium conditions. In order to access the time characteristics of the charge dynamics, pulsed optical excitation with the setup of chapter 2.2 is applied.

First measurements of time spectra of the optically induced signal at the n-doped GaAs surface were taken for increasing tunnel currents. In contrast to the work of Shigekawa et al., a significant current dependency is discovered. For increasing tunneling rates the decay time of the optically induced current shortens considerably. We interpret this observation with the hole annihilation process via tunneling electrons from the STM tip into the photo-generated holes. Naturally, this process occurs faster at higher tunnel currents.

If the tunneling rate is increased even more, a surprising change in the time spectra can be observed. Apart from the fast decay, attributed to the hole annihilation mechanism, a slower contribution in the spectra becomes visible. The characteristic decay time of this second process shows only little dependency on the tunnel current.

We explain the emergence of the second process at higher currents with drastic non-equilibrium conditions inside the tip-induced space charge region. The relaxation in this case is defined by two mechanisms: the hole recombination and the re-ionization of surface near dopants. The first mechanism can be controlled via the tunnel current whereas the time characteristic of the second one is intrinsic. When driving the hole annihilation process to very short decay times by increasing the tunneling rate, for a complete relaxation the slower ionization process of the donors has still to occur. The time characteristics of this ionization are visible in the time spectra at high currents.

In order to investigate the dopant dynamics in more detail we performed a spatiotemporal analysis of the ionization process for three donors situated at different depths beneath the surface. It becomes clearly visible that the ionization occurs on faster time scales for deeper dopants. As thermal emission can be ruled out as the mechanism we propose a field-driven ionization process. Previous studies have shown a significant increase in the binding energy for donors near the surface. In a first approximation this is able to explain our variation in the ionization time for the three dopants.

However, when modeling the ionization process, it is found, that not only the variation in the binding energy of the donors plays a crucial role for the ionization time. In fact, the local 
configuration of dopants is more fundamental. When all holes are annihilated via the tunnel current and all surface-near donors are neutral, the surface is free of electric fields and ionization becomes very unlikely. In order to rebuild the tip-induced space charge region, first, locally deep donors ionize as they have the smallest binding energy. Then, the newly induced electrostatic field triggers the ionization of further dopants nearer to the surface and so on. As a consequence, the local distribution of donors has a massive impact on the ionization dynamics or more drastically: every donor has its unique ionization time, very dependent on its local surrounding.

\section{Original publication}

The key elements in the steady miniaturization process of cutting-edge semiconductor devices are the understanding and controlling of charge dynamics on the atomic scale. In detail, this addresses the study of charging processes of individual doping atoms and especially the interaction with their surrounding. Here, we utilize pulsed optical excitation in combination with Scanning Tunneling Microscopy at the n-doped $\operatorname{GaAs}(110)$ surface to investigate single donor dynamics within a nanoscaled, localized space charge region. Tuning the tunnel rate can drive the system into non-equilibrium conditions, allowing to distinguish between the decay of optically induced free charge carriers and the decay of donor charge states. The latter process is atomically resolved and discussed with respect to donor level binding energies and local donor configurations.

P. Kloth, K. Kaiser and M. Wenderoth, Science Advances 3:e1601552 (2017)

\section{Introduction}

Optical excitation of a semiconductor generates electron-hole pairs. Besides simple recombination, the presence of local fields and structural inhomogeneities can result in complex relaxation pathways ${ }^{102}$. This includes field-driven charge transport but also the interaction with local defects. Up to now, access to the correspondent microscopic processes is usually limited to probing a large volume of the host material ${ }^{103,104}$. On the macroscopic scale, a standard indicator, characterizing dynamic properties of charge processes inside a semiconductor, is the Surface Photovoltage (SPV). The SPV describes the change of local potentials due to the presence of photo-generated charge. Microscopically, the build-up of an SPV is linked to various mechanisms such as the dynamics of dopants and the optically excited, freely movable carriers within the semiconductor. Especially one process, the controlled charging and discharging of individual dopants, is the most elementary process in semiconductor electronics. In state-of-the-art device development, switching is already accomplished by a handful of donors ${ }^{1,105,106}$. In this context, disorder, e.g. the specific configuration of doping atoms, is crucial. SPV studies have already reported a significant inhomogeneity of the static properties ${ }^{11,15}$ on the atomic scale. Apart from its relevance in fundamental research, an atomic level view on charge dynamics is needed for further device miniaturization. In particular, 
determining the influence of boundaries, as e.g. surfaces or internal interfaces, will substantially improve our understanding of dynamic properties of semiconductor nanostructures.

$$
\text { A }
$$

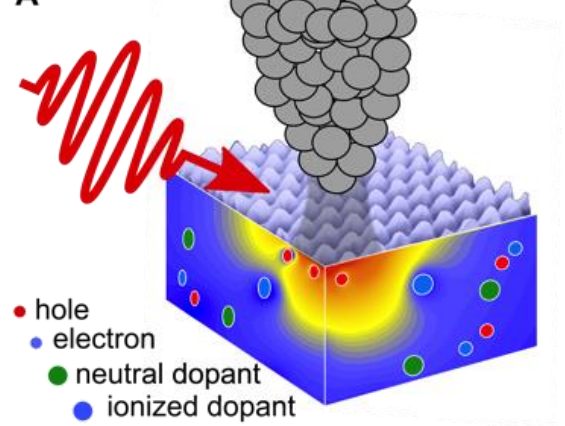

- ionized dopant

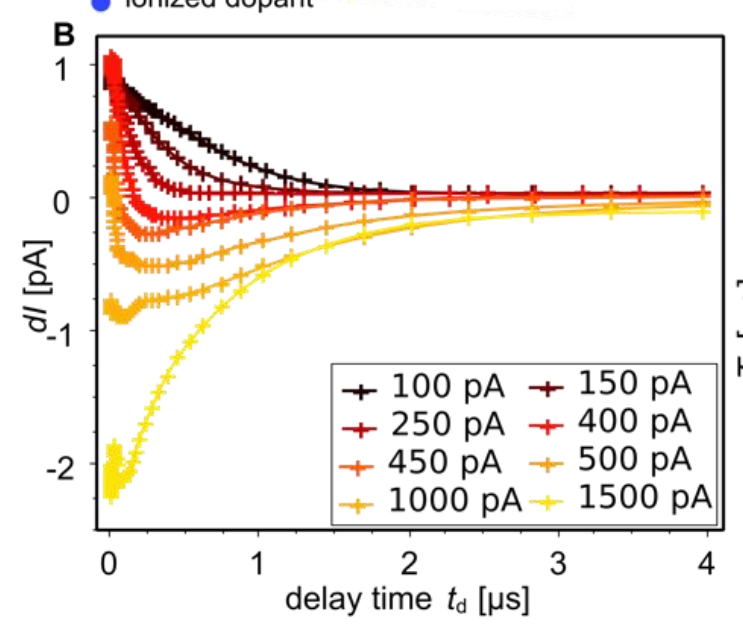

C

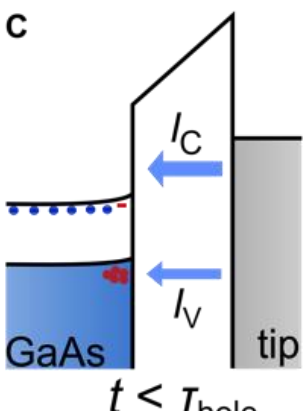

D
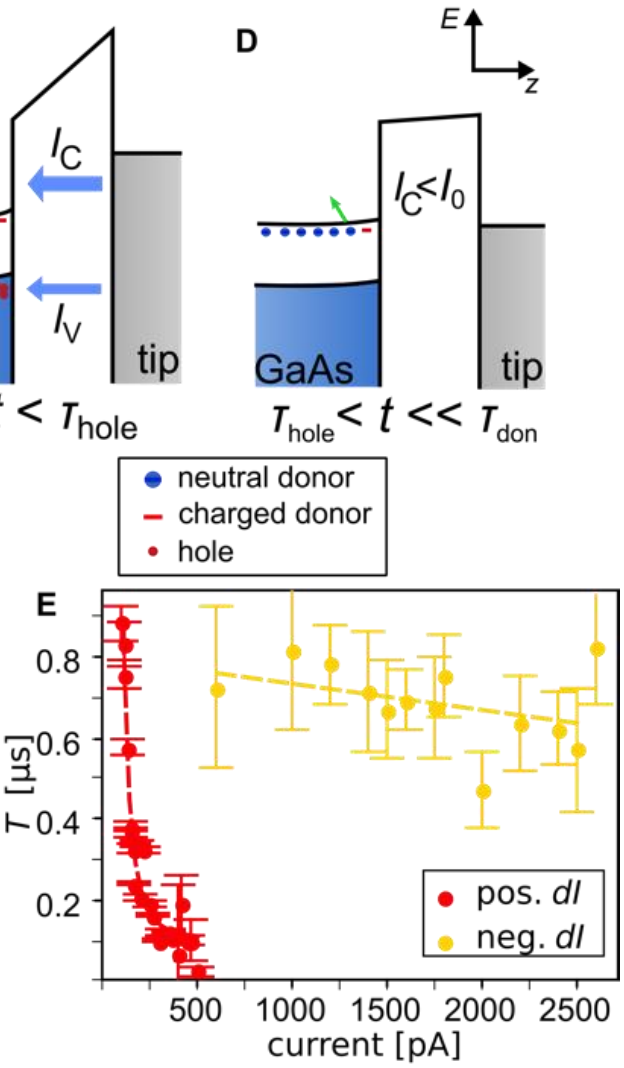

Fig. 4.7 Charge dynamics at the GaAs(110) surface. (a) The STM tip induces a space charge region below the $\mathrm{GaAs}(110)$ surface, leading to the separation of photo-generated electron-hole pairs. The presence of holes beneath the tip modifies the charge configuration at the surface. (b) The optically induced current $d l$ as function of the delay time $t_{\mathrm{d}}$ reveals an altering sign from positive to negative values going from low to high tunnel currents (bias voltage $1.0 \mathrm{~V}$, exc. parameters: $8 \mu \mathrm{s}$ repetition cycle, $40 \mathrm{~ns}$ pulse width, $10 \mu \mathrm{W}$ average power). (c) Band tunneling scheme for $t<T_{\text {hole. }}$. At low tunnel currents, $d /\left(t_{\mathrm{d}}\right)$ is dominated by the hole annihilation process at the surface via $I_{v}$. (d) Band tunneling scheme for $T_{\text {hole }}<t<T_{\text {don. }}$. For high currents, holes at the surface are annihilated fast, while surface-near donors still have to emit their electrons into the conduction band in order to ionize (green arrow). Nearly no net charge at the surface is present leading to a vanishing $I_{c}$. Please note that in (c) and (d) the same bias voltage is applied. (e) Decay constant $\tau$ plotted against current $I_{0}$ in case of positive and negative $d l$. The dashed lines are intended as a guide to the eye.

Here, we demonstrate the necessity for this atomically scaled analysis of locally very inhomogeneous charging dynamics of individual donors, which are up to now, usually described by a single decay constant. We have used an optical pump-probe technique combined with Scanning Tunneling Microscopy (STM) (Fig. 4.7a and Fig. 4.10), by applying the shaken-pulse-pair excitation (SPPX) $)^{24,25,64}$ procedure. The time evolution is measured by a periodic modulation of the optically induced tunnel current $d I$ as function of the delay time $t_{\mathrm{d}}$ between pump and probe pulse. Our sample system, sketched in Fig. 4.7a, is the STM tip-induced electrostatic potential (colorcoded) ${ }^{107}$ in n-doped (silicon doping) GaAs, including the influence of optically excited electronhole pairs,. Comparable to the situation inside a Schottky contact and leading to a SPV, the electric field separates free charge resulting, at positive bias voltages, in an accumulation layer of holes at 
the surface. In return, initially ionized donors capture electrons and become neutral. The steady state of the charge configuration is defined by the relaxation mechanisms of the donors and the electron-hole pairs plus their mutual interaction ${ }^{4,108}$. Additionally, preliminary studies have shown ${ }^{18}$, that tunneling electrons can directly annihilate the photo-generated and accumulated holes at the surface. This allows us to use the tunnel current $I_{0}$ as an additional control parameter to drive the system into different non-equilibrium conditions (see also chapter 3.3).

\section{Results}

As a unique feature and in contrast to previous studies ${ }^{25,64}$, we apply low laser intensities $P_{\mathrm{L}}$. Fig. 4.7b shows a multitude of $d I\left(t_{\mathrm{d}}\right)$ spectra for increasing $I_{0}{ }^{25}$. At $I_{0}$ smaller than $250 \mathrm{pA}$, we observe an exponential decay of $d I\left(t_{\mathrm{d}}\right)=I_{0} \cdot \exp \left[-(1 / \tau) \cdot t_{\mathrm{d}}\right]$ with a decay constant $\tau$ decreasing with increasing $I_{0}$ (Fig. 4.7e, red dots). We attribute this $I_{0}$ dependency of $\tau$ to the tunnel process into photo-generated holes (see $I_{\mathrm{V}}$ in Fig. 4.7c) inside the valence band ${ }^{17,18}$. Please note, that the size of the SCR changes when decreasing the tip-sample distance for increasing $I_{0}$. However for a tip movement of 1-2 $\AA$ (current range in Fig. 4.7b for a usual tunnel barrier height of $4 \mathrm{eV}$ ) this effect is negligible (see Fig. 4.13). Consequently, in this current regime the decay of $d I\left(t_{\mathrm{d}}\right)$ gives access to the annihilation process of the accumulated holes at the surface. By adjusting $I_{0}$, we are able to actively control this decay, in the following termed as $\tau_{\text {hole }}$.

At $I_{0}=400 \mathrm{pA}$ the line shape of $d I\left(t_{\mathrm{d}}\right)$ changes notably. Although $d I$ remains positive for small $t_{\mathrm{d}}$ and decays fast, it alters its sign for larger $t_{\mathrm{d}}$. This effect gets even more pronounced for higher currents $(450 \mathrm{pA}, 500 \mathrm{pA})$ until a completely negative and exponentially decaying $d I\left(t_{\mathrm{d}}\right)$ is observed $(1000 \mathrm{pA}, 1500 \mathrm{pA})$. We explain the line shape of $d I\left(t_{\mathrm{d}}\right)$ at higher currents with a crossover between two relaxation processes. The hole annihilation dominates at low and medium $I_{0}$ (Fig. 4.7c). The negative $d I$ can be identified as the signature of donor ionization by electron emission from the donor level into the conduction band (green arrow in Fig. 4.7d). During the relaxation to the ground state, $d I\left(t_{\mathrm{d}}\right)<0$ reflects a special non-equilibrium condition. For high $I_{0}$, holes are annihilated rapidly whereas donors remain neutral. Consequently, nearly no net charge is found at the surface, the potential difference between tip and sample at the vacuum barrier vanishes and the tunnel current drops almost to zero (Fig. 4.7d). Most important, the relaxation of the system (at $\tau_{\text {hole }}<t<\tau_{\text {don }}$ ) is solely determined by the ionization of the donors (Fig. 4.14). As expected for this mechanism, the related decay constant $\tau_{\mathrm{don}}$ is significantly less dependent on $I_{0}$ in comparison to $\tau_{\text {hole }}$ (Fig. 4.7e yellow dots, exemplary fitting results can be found in Fig. 4.15 and Fig. 4.16).

Expecting a great influence of different atomically scaled donor configurations at the GaAs surface, we have studied $\tau_{\text {don }}$ locally resolved. The constant current topography in Fig. 4.8a shows the signatures of three donors, positioned at different depths beneath the surface. In order to suppress the contribution of the hole dynamics, we use high tunnel currents $\left(I_{0}=1 \mathrm{nA}\right)$ while recording $d I\left(t_{\mathrm{d}}\right)$ spectra at each raster-scan point of the topography in Fig. 4.8a. Additionally, we have chosen a 
sample voltage $\left(U_{\mathrm{B}}=1.3 \mathrm{~V}\right)$ where lateral interaction between donors can be neglected. Single, time-resolved spectra, taken directly at the marked dots, clearly show significantly different decay constants for each donor (Fig. 4.8b, logarithmic scale analysis: Fig. 4.17). Although it was already shown that local defects have an impact on the relaxation process ${ }^{25,64}$, a considerable variation in the dynamics of individual defects have not been resolved yet. To explore this, we plot $\tau_{\text {don }}$ spatially resolved in Fig. 4.8c (a movie showing the spatiotemporally resolved decay of $d I\left(t_{\mathrm{d}}\right)$ can be found in the supplementary material). Clearly visible is a local enhancement of $\tau_{\mathrm{don}}$ at the donor position. Moreover, $\tau_{\mathrm{don}}$ changes from donor to donor with the highest $\tau_{\mathrm{don}}$ for donors closest to the surface. Compared to the free surface (i.e. far away from a visible donor) showing a $\tau_{\text {don }} \sim 100 \mathrm{~ns}$, a donor in layer 1 below the surface exhibits a $\tau_{\text {don }}$ of $530 \mathrm{~ns}$.
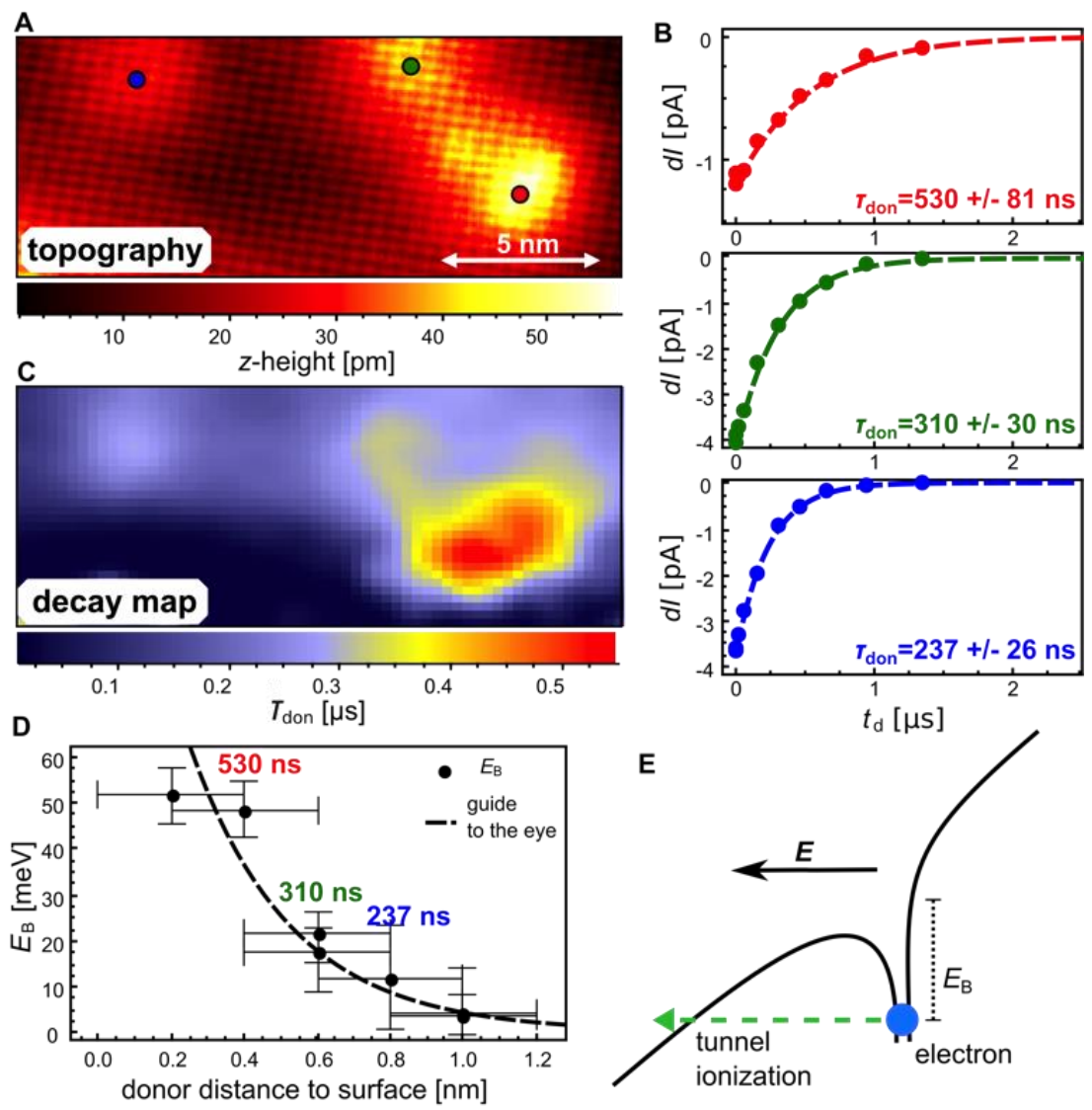

$E$

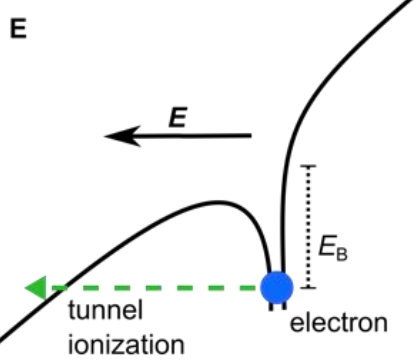

Fig. 4.8 Spatially resolved charging dynamics of donors at different depths below the GaAs surface. (a) Topography of the GaAs(110) surface with three donors (marked as red, green and blue dots) positioned at different depths below the surface (bias voltage $1.3 \mathrm{~V}$, tunnel current $1 \mathrm{nA}$ ). (b) Locally resolved time spectra (bias voltage $1.3 \mathrm{~V}$, tunnel current $1 \mathrm{nA}$, exc. parameters: $8 \mu$ s repetition cycle, $40 \mathrm{~ns}$ pulse width, $16 \mu \mathrm{W}$ average power) recorded at the positions marked in (A). (c) Spatially resolved decay time $T_{\text {don. }}$ (d) Donor binding energies $E_{\mathrm{B}}$ plotted versus depths. Adapted from ${ }^{109}$. $T_{\mathrm{don}}$ of the corresponding donors in (b) is assigned respectively. (e) Model of field ionization. In this case, electrons tunnel from the donor level into the unoccupied conduction band states.

Obviously, the surrounding of the defects plays a crucial role, e.g. the presence of the surface and/or the influence of other donors. In this sense, the absolute values of $\tau_{\text {don }}$ will depend on the local dopant configuration. To elucidate the variation in $\tau_{\text {don }}$ in $\mathbf{F i g . ~} \mathbf{4 . 8}$, we analyze the emission mechanism of the ionization process in detail. By performing a temperature-dependent study, 
thermal emission can be ruled out (see Fig. 4.19). Previous results ${ }^{109}$ have shown, that $E_{\mathrm{B}}$ monotonically increases with decreasing depth of the donor (Fig. 4.8d and see Fig. 4.18). Based on this, we propose a field-driven tunneling process of electrons from the donor level to the conduction band (Fig. 4.8e) to explain the correlation between $E_{\mathrm{B}}$ and $\tau_{\mathrm{don}}(\mathbf{F i g}$. 4.8d).

The electron emission rate for this tunneling process is determined by the binding energy $E_{\mathrm{B}}$ as well as the local field $\boldsymbol{E}$ at the donor position. To cross-check this $E_{\mathrm{B}}$ dependency, we compare the experimental $\tau_{\text {don }}$ quantitatively with the ionization times $\tau_{\text {hydro }}$ calculated for hydrogen atoms in an electric field ${ }^{110}$ with adapted parameters for silicon donors in bulk GaAs (Fig. 4.9a, see also Fig. 4.20). For comparison, the found pairs of $E_{\mathrm{B}}$ and $\tau_{\text {don }}$ for the three different dopant depths below the surface in Fig. 4.8a are included in Fig. 4.9a. The relaxation from the non-equilibrium conditions at high currents $I_{0}$ is characterized by the presence of nearly no charge and hence a weak electric field at the surface (Fig. 4.7d). Charge fluctuations of donors and thereby field fluctuations take place ${ }^{19,111}$, triggering further field-driven ionizations. We model an electrostatic potential in a cross-section at the GaAs surface (Fig. 4.9b, c), including random donor positions in charged (blue dots) or neutral (green dots) state, according to the doping density of our sample $\left(3 \cdot 10^{18} \mathrm{~cm}^{-3}, 7 \mathrm{~nm}\right.$ average distance between two donors). In order to estimate the probability of charging events, we calculated exemplary Coulomb potentials of ionized donors in this half space geometry.

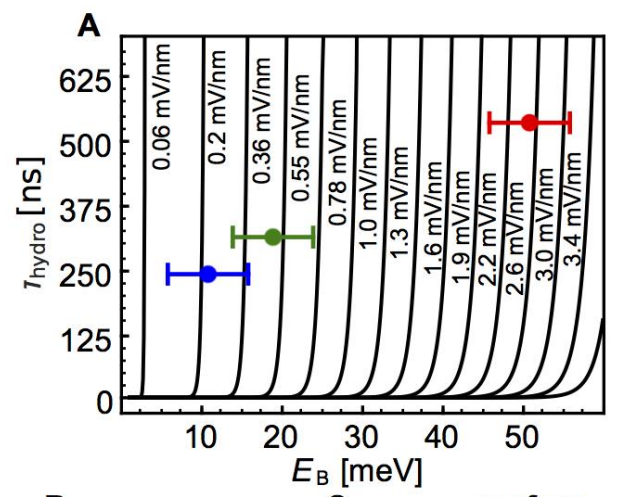

Fig. 4.9 Comparison of donor dynamics: theory versus experiment. (a) Calculated field-driven ionization time $T_{\text {hydro }}$ for a hydrogen model with adapted parameters, plotted against the binding energy $E_{\mathrm{B}}$ and compared with experimental pairs of $E_{\mathrm{B}}$ and $\tau_{\text {don }}$. $(b, c)$ Calculated snapshots of the electric field $|E|$ induced by an exemplary ionized donor in crosssectional geometry at the GaAs surface, based on a random distribution of donors, charged (blue) and noncharged (green). The color scale is cut at $5 \mathrm{mV} / \mathrm{nm}$. The volume, marked by the white contour lines, gives the

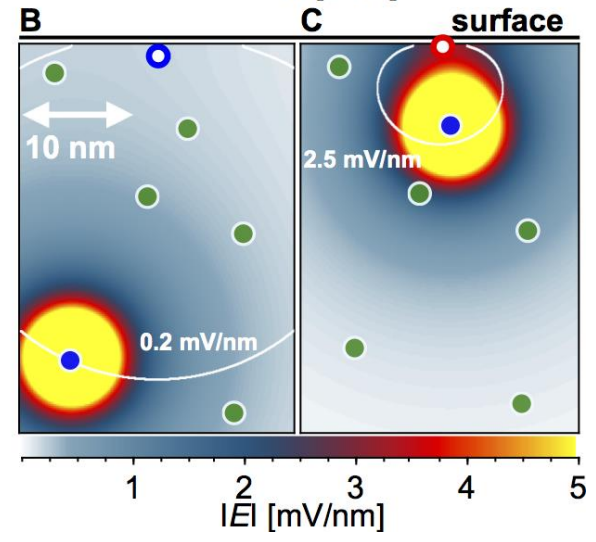
maximum distance for a charged donor in which the donor is able to trigger further ionization of surfacedonors with $E_{\mathrm{B}}=10 \mathrm{meV} / T_{\text {don }}=237 \mathrm{~ns}(\mathrm{~B}$, blue/white dot) or $E_{\mathrm{B}}=45 \mathrm{meV} / T_{\text {don }}=530 \mathrm{~ns}(\mathrm{C}, \mathrm{red} /$ white dot).

Regarding the ionization process of donors with a binding energy $E_{\mathrm{B}}$ of $10 \mathrm{meV}$ and a $\tau_{\mathrm{don}}$ of $237 \mathrm{~ns}$ (blue/white dot, Fig. 4.9b), the required field $\boldsymbol{E}$ of $0.2 \mathrm{mV} / \mathrm{nm}$ will already be given, if any of the donors in a spherical volume marked by the white contour line in Fig. 4.9b, ionizes. In contrast, in order to charge a donor with a binding energy $E_{\mathrm{B}}$ of $45 \mathrm{meV}$ and a $\tau_{\mathrm{don}}$ of $530 \mathrm{~ns}$ (red/white dot, 
Fig. 4.9c) a field $E$ of $2.5 \mathrm{mV} / \mathrm{nm}$ is needed. The radius of the correspondent volume reduces to $8 \mathrm{~nm}$ (white contour line, Fig. 4.9c) and consequently, the number of possible donor-candidates decreases, making the ionization process unlikely. We deduce, that the variation in $E_{\mathrm{B}}$ cannot be the only reason for the variation of $\tau_{\text {don }}$. In fact, we expect a considerable increase of the ionization probability when more than one donor is involved. From this, we propose a relaxation to the ground state as stepwise build-up of the electric field, starting with the ionization of donors positioned in the bulk material and continuing to donors near the surface. Hence, the donor dynamics cannot be treated as single charging and discharging processes, connected to a dedicated decay constant $\tau_{\text {don }}$. Instead they have to be regarded as a stochastically distributed ensemble of linked subsequent charging events triggered by random charge fluctuations. Thus, the actual relaxation dynamics will vary from one local donor configuration to another.

\section{Discussion}

We are convinced, that this coupled charging dynamics of individual donors including the influence of surfaces and interfaces can have massive impact on the functionality of semiconductor devices. Bi-stabilities in the charge state of coupled donors have already been observed in the millisecond time regime ${ }^{19}$. The map for the relaxation time of individual donors in Fig. 4.8c shows non-isotropic local enhancements of $\tau_{\mathrm{don}}$, indicating the interaction among themselves. Continuous charging and discharging events are made responsible for shot noise in semiconductor devices ${ }^{112}$, but are not yet experimentally resolved on the local scale. Even for quantum computer approaches, single donors as isolated qubits are discussed ${ }^{113-115}$. Ultimately, in all these subjects a detailed understanding can only be gained by atomic scale characterization. In this context pulsed optical excitation combined with STM opens an exciting new research field with a myriad of applications.

\section{Supplemental material}

\section{S4.1: Experimental setup, methods and sample system}

All data were recorded in a home-built low-temperature, ultra high vacuum Scanning Tunneling Microscope (STM) at a base pressure of $10^{-10} \mathrm{mbar}$ and at a base temperature of $77 \mathrm{~K}$ and $6 \mathrm{~K}$. The bias voltage is defined as a sample voltage. The silicon n-doped GaAs $\left(3 \cdot 10^{18} / \mathrm{cm}^{3}\right)$ crystals were chemically thinned down to $100 \mu \mathrm{m}$ and cleaved inside the vacuum chamber exposing a (110)surface. The optical setup implemented in the STM is sketched in Fig. 4.10. The laser beam of a continuous-wave laser diode (Toptica, $785 \mathrm{~nm}$ ) is processed into pulsed shape by a fiber-coupled electro-optic modulator (EOSpace). We use the shaken-pulse-pair excitation (SPPX), conceived in the group of Hidemi Shigekawa at the University of Tsukuba. A high frequency arbitrary waveform generator (AWG, Keysight 81160A) produces the SPPX in an all-electronic method. The shortness of the pulses is restricted by the bandwidth of the AWG to $\sim 1 \mathrm{~ns}$. The pulsed laser 
beam is focused into the tunnel junction with a focus diameter of less than $20 \mu \mathrm{m}$. The periodically modulated, laser-induced tunnel signal $d I\left(t_{\mathrm{d}}\right)$ is measured by lock-in amplification.

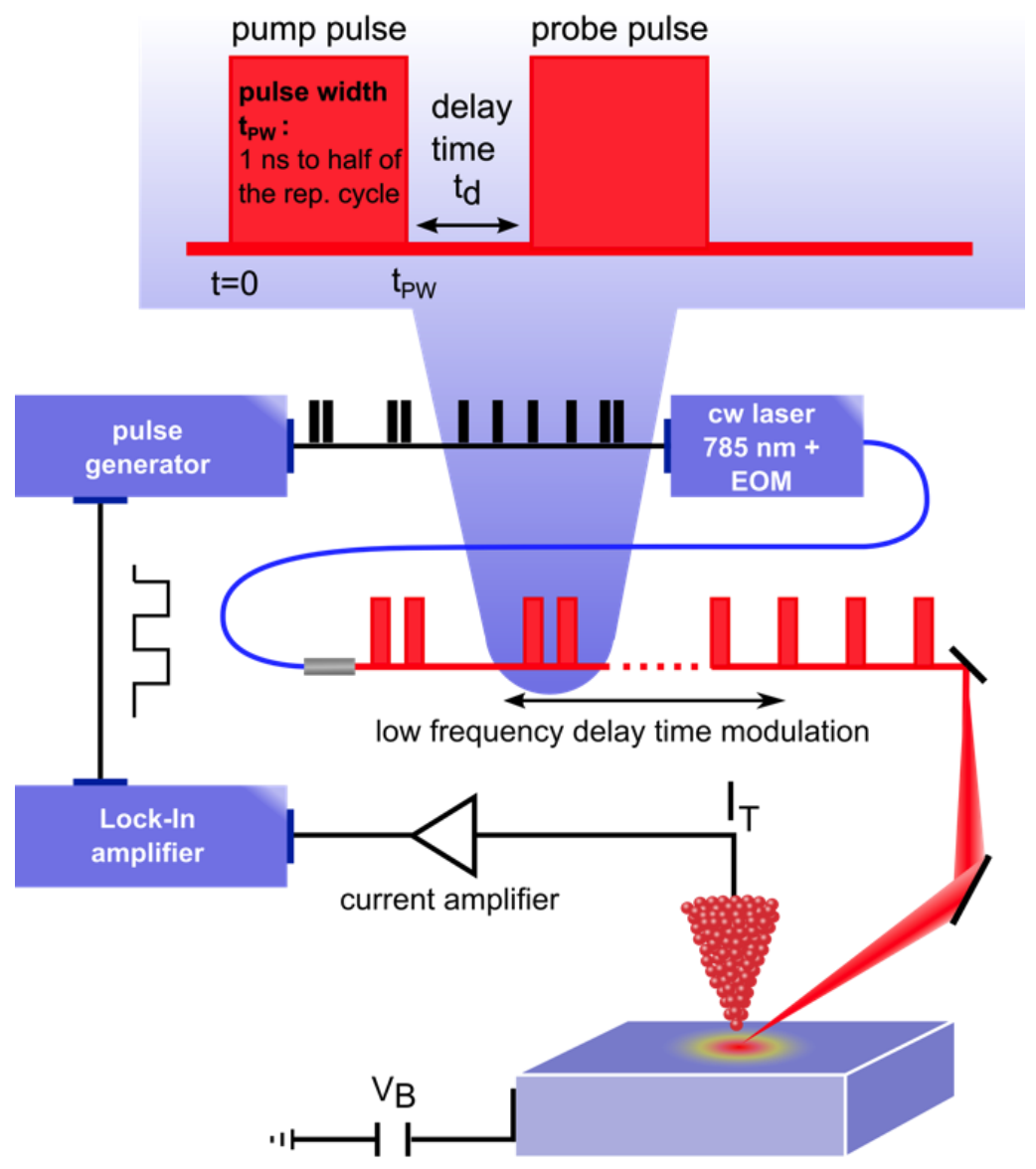

Fig. 4.10 Optical pulse generation for STM operation. The laser beam of a low-noise diode laser (100 mW, continuous wave, $785 \mathrm{~nm}$ ) is processed into pulsed shape with the help of an electro-optic modulator (EOM), controlled by a high frequency pulse generator. After focusing the pulses into the tunnel junction, the optically induced signal is extracted via lock-in amplification.

\section{S4.2: Excitation state dependent sign of the optically induced current $d I\left(t_{\mathrm{d}}\right)$}

The quantity $d I\left(t_{\mathrm{d}}\right)$ is the difference in the tunnel current induced by a sequence of two shortly separated pulses compared to the current induced by a train of two well-separated pulses. In comparison to other pump-probe experiments the pump as well as the probe pulse have the identical intensity and both trigger the same mechanism: a change in the tunnel current.

In contrast to our studies, $d I$ in previous optical pump-probe SPV measurements had a negative sign. We can show, that the sign of $d I$ is determined by different excitation states after the pump pulse $\left(t=t_{\mathrm{PW}}\right)$. Fig. 4.11a pictures schematically two shortly separated optical pulses (green), discussing a saturated (Fig. 4.11b, blue) and a non-saturated excitation case (Fig. 4.11c, red) and compares this with the excitation induced by two well separated double pulses (Fig. 4.11d). 

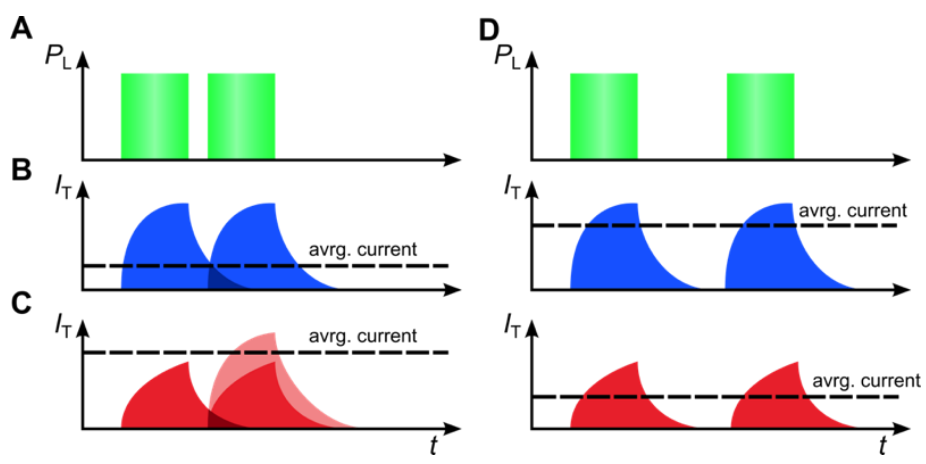

Fig. 4.11 Sign reversal in $d$, dependent on the excitation state of the system after the pump pulse. (a) Two shortly separated optical pulses. (b) Response in the tunnel current when the system has reached its local equilibrium after the pump pulse. (c) Response in the tunnel current when local equilibrium is not reached. (d) System response in case of well-separated pulses. The dashed lines mark the average current for each pulse configuration.

If the pump pulse has induced the maximum change in the tunnel current $I_{\mathrm{T}}$ (Fig. 4.11b), the effect of the probe pulse on the tunnel current will be at maximum of the same order. Due to the overlap in the decay (deep blue shading), the average current is less in comparison to the average current (black dashed lines) induced by two well-separated pulses (Fig. 4.11d). Per definition this leads to a negative $d I$.

If the first pulse does not induce the maximum effect, the shape of the system response, triggered by the second pulse, can change considerably. Fig. 4.11c illustrates the configuration, for which the excitation by the probe pulse leads to more tunnel current (light red shading) in comparison to one single, isolated pulse. In the differential measuring method of the averaged signal (black dashed line) this may lead to positive $d I$.

A

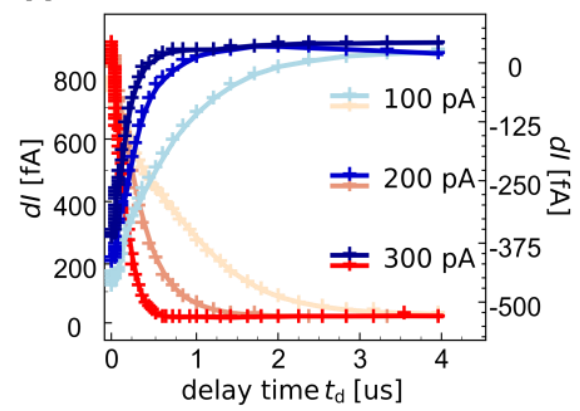

B

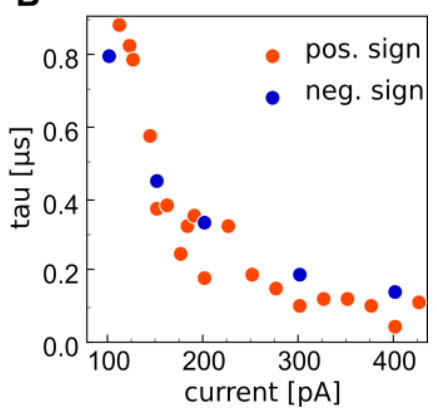

Fig. 4.12 $d I\left(t_{\mathrm{d}}\right)$ spectra for low and high optical excitation intensity. (a) At low optical excitation intensity $P_{\mathrm{L}}$, saturation of the SPV during the pump pulse is not obtained resulting in a positive $d l$ (red curves). At high $P_{\mathrm{L}}$, saturation of the SPV is obtained after the pump pulse (blue curves). Consequently the $d l$ becomes negative.

(b) The current dependency of $T_{\text {hole }}$ is in both cases identical.

Experimentally, saturation (Fig. 4.11b) or non-saturation (Fig. 4.11c) in the excitation state can be investigated by applying different laser intensities $P_{\mathrm{L}}$ (Fig. 4.12a, red curves: low excitation intensity, blue curves: high excitation intensity). The time to obtain saturation for the charge configuration at the surface is connected to the time it takes for a donor to capture one of the photo- 
generated electrons and to neutralize subsequently. At higher $P_{\mathrm{L}}$ the electron density at the surface is denser and consequently the Surface Photovoltage (SPV) builds-up faster during the pump pulse.

The current dependency of the decay constants $\tau_{\text {hole }}$ extracted from $d I\left(t_{\mathrm{d}}\right)$ spectra are identical In (Fig. 4.12b). This implies that in each case, the same relaxation mechanism is probed, namely the annihilation of the holes via tunneling. The only difference in both cases is the excitation state of the charge configuration at the surface after the pump pulse, resulting in the sign alteration of $d I$.

\section{S4.3: Evolution of the Space Charge Region when varying the z-height of the STM tip}

In Fig. 4.7b in the main text, the change of $d I\left(t_{\mathrm{d}}\right)$ spectra is discussed for increasing tunnel currents. For a current change from 150 to $2500 \mathrm{pA}$, the STM tip approaches 1-2 $\AA$ towards the sample surface (assuming a usual tunnel barrier height of $4 \mathrm{eV}$ ). With this variation in the tip height, also a variation in the tip-induced potential can be expected. Fig. 4.13a-c shows the evolution of the SCR when the tip height above the surface is changed from 8 to $6 \AA$ (doping density $n_{\mathrm{D}}=3 \cdot 10^{18} / \mathrm{cm}^{3}$, sample voltage $1.0 \mathrm{~V}$, tip radius $30 \mathrm{~nm}$ ). For this analysis, we used a commercial Poisson solver (COMSOL). Qualitatively, the size of the SCR varies only little.
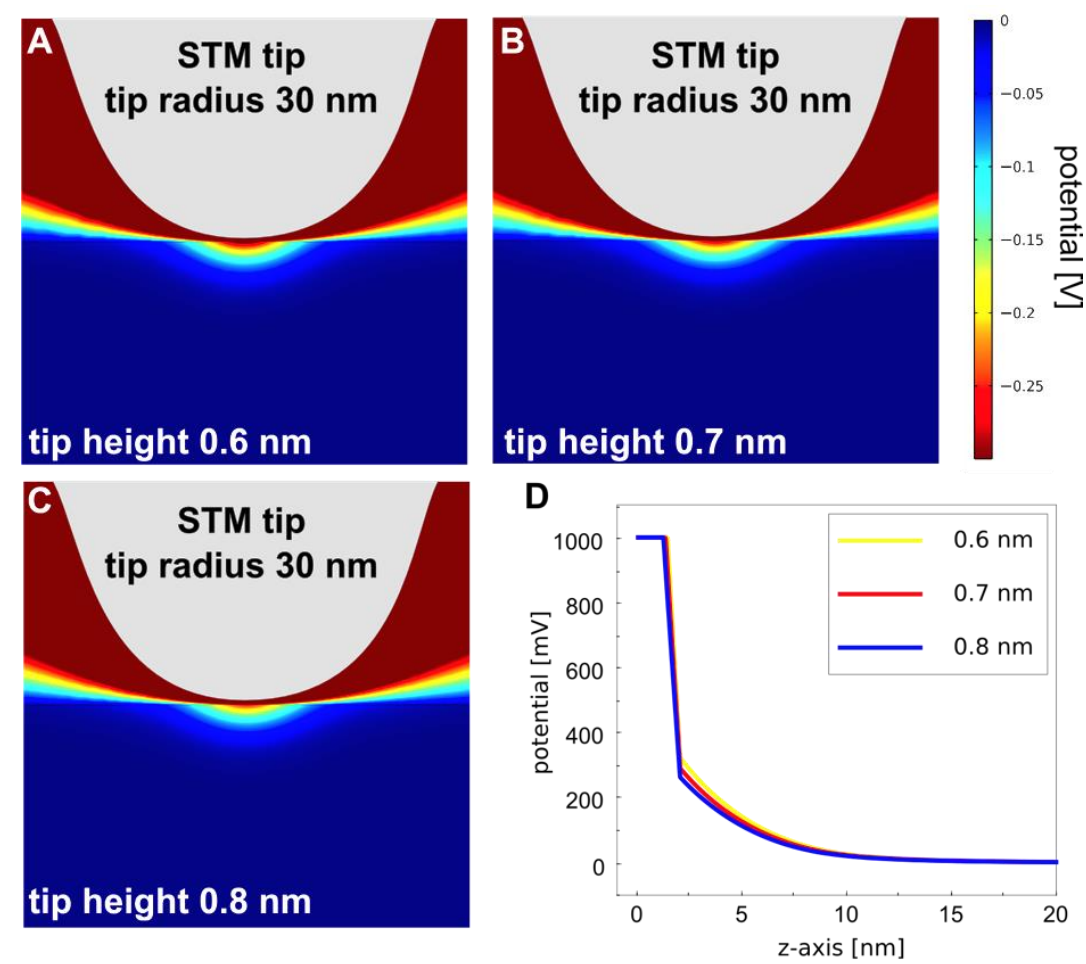

Fig. 4.13 Calculation (Poisson solver) of the tip-induced potential for varying tip heights above the GaAs surface. (a-c) Spatial resolved potential (plotted color coded) for tip height of $0.6,0.7$ and $0.8 \mathrm{~nm}$ (doping density $n_{\mathrm{D}}=3 \cdot 10^{18} / \mathrm{cm}^{3}$, sample voltage $1.0 \mathrm{~V}$, tip radius $30 \mathrm{~nm}$ ). The color scale is cut at $-0.3 \mathrm{~V}$ for a better visibility. (d) Cross-sectional plot of the potential along the $z$-axis and in the middle of the STM tip.

For a more quantitative comparison, Fig. 4.13d demonstrates the change of the SCR in a crosssectional line profile of the induced potential along the $z$-axis starting at the center of the STM tip. The spatial extension of the SCR is for all heights roughly $10 \mathrm{~nm}$. The absolute tip-induced 
potential at the surface varies, when going from 6 to $8 \AA$, only by $60 \mathrm{mV}$. Concluding, it can be stated, that the $z$-movement of the STM tip for the data in Fig. 4.7b is negligible.

\section{S4.4: Detecting the donor ionization process}

In case of high currents $I_{0}$, photo-generated holes at the surface decay fast, leading to a small decay constant $\tau_{\text {hole }}$. If the relaxation time of the donors is longer, the imbalance between $\tau_{\text {hole }}$ and $\tau_{\text {don }}$ will lead to interesting charge configurations. In the following we discuss the time evolution of the hole and ionized donor concentration upon and after pulsed excitation combined with the corresponding tunnel currents $I_{\mathrm{V}}$ and $I_{\mathrm{C}}$ (Fig. 4.14a) and screening conditions sketched in tunneling band schemes (Fig. 4.14b-e).
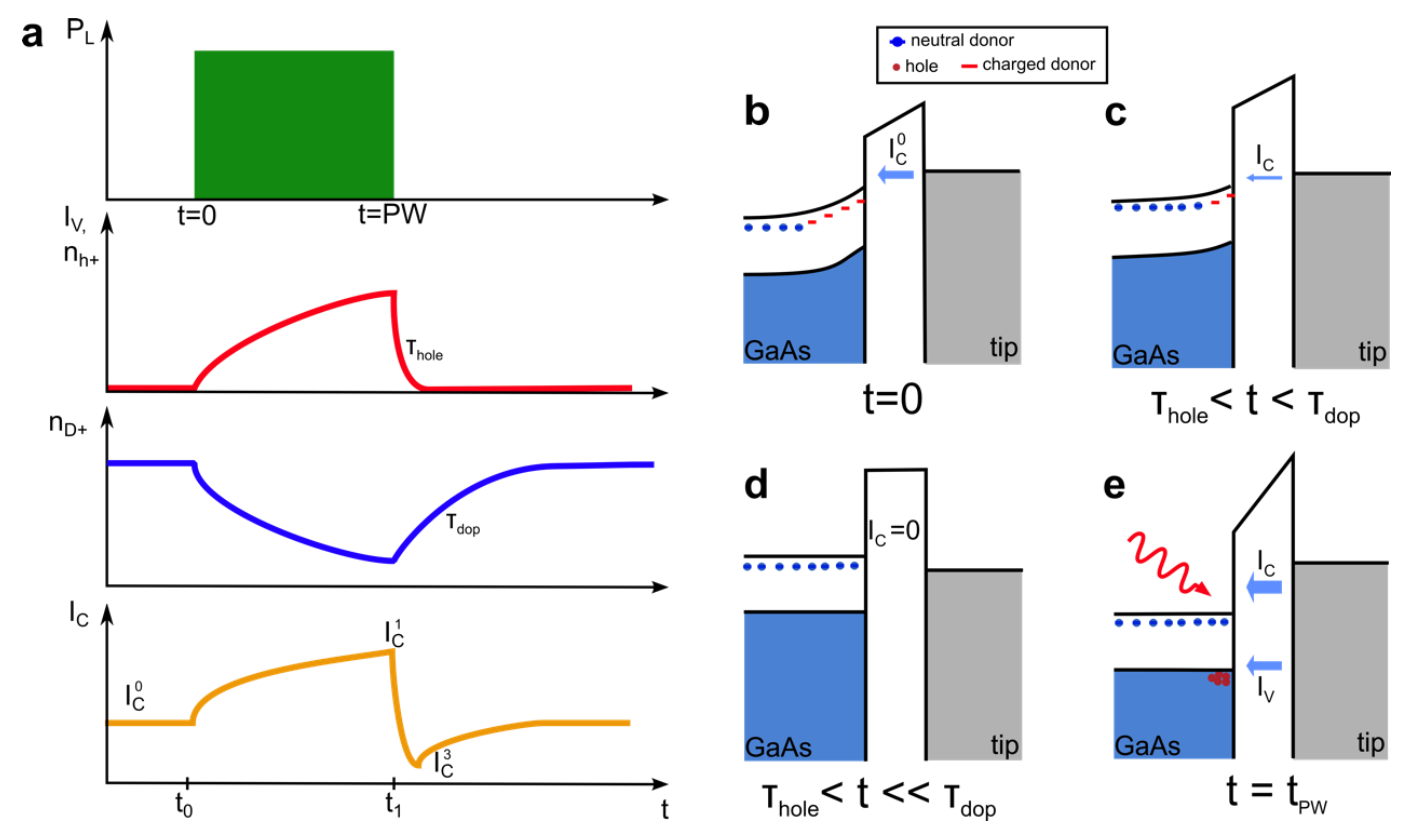

Fig. 4.14 Real time evolutions of important experimental parameters and the corresponding screening configurations sketched in band schemes. (a) Laser intensity $P_{\mathrm{L}}$, hole concentration (valence band tunneling) $n_{h+}, I_{V}$, concentration of ionized donors at the surface $n_{D_{+}}$and conduction band tunneling $I_{C}$ plotted in real time in case of fast hole annihilation. (b-e) Screening configurations at characteristic time points sketched in tunneling band schemes.

Under dark conditions (Fig. 4.14b, $t=0$ ) tip-induced band bending with the charge provided by ionized dopants is found. The conduction band tunneling $I_{\mathrm{C}}{ }^{0}$ is given by the set point. Upon excitation $\left(0<t<t_{\mathrm{PW}}\right)$ holes accumulate at the surface increasing the hole concentration $n_{\mathrm{h}+}$ and leading to valence band tunneling $I_{\mathrm{V}}($ Fig. 4.14c). Simultaneously, the concentration of ionized dopants $n_{\mathrm{D}+}$ decreases by electron capturing and the more efficient screening of the tip potential leads to an increase of $I_{\mathrm{C}}{ }^{1}$ (Fig. 4.14c). At $t>t_{\mathrm{PW}}$ for high currents, $I_{\mathrm{V}}$ and also $n_{\mathrm{h}+}$ decay fast. Under extreme conditions, all holes at the surface have disappeared but yet no dopant has ionized (Fig. 4.14d). Consequently charge is missing at the surface and during these temporary nonequilibrium conditions the voltage drop over the vacuum barrier gets small and likewise the conduction band tunneling vanishes $\left(I_{\mathrm{C}}{ }^{3}=0\right)$ resulting in a negative $d I$. Eventually the remaining 
neutral donors at the surface ionize and the system relaxes back to its ground state (Fig. 4.14e). The exponential decay for negative $d I$ gives us access to the time characteristics of these donor charging events.

\section{S4.5: Exemplary fitting results of $d I\left(t_{\mathrm{d}}\right)$ curves of Fig. $4.7 \mathrm{e}$}

In order to extract the current-dependency of the $d I\left(t_{\mathrm{d}}\right)$ spectra, shown in Fig. 4.7e, we conducted exponential fitting routines. Two exemplary results for low and high tunnel currents are shown in

Fig. 4.15.
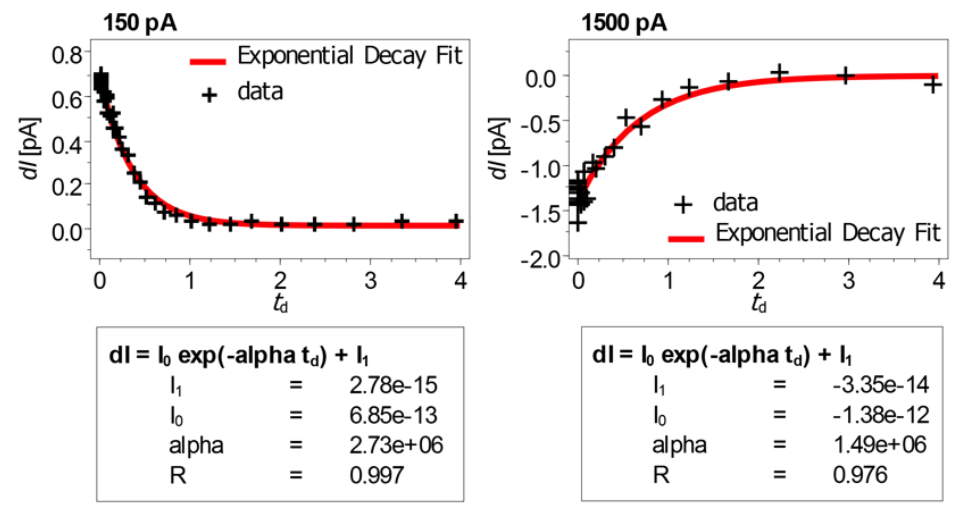

Fig. 4.15 Two exemplary fitting results of the $d l\left(t_{\mathrm{d}}\right)$ spectra of Fig. 4.7e. The parameter $R$ stands for the nonlinear regression coefficient. The other parameters are self-descriptive.

Please note, that in these two spectra one isolated decaying process is visible. In the transition region (450-550 pA) we applied fittings of single exponential decaying function. In this case, we restricted the fitting region to correspondent early and late decay time points $t_{\mathrm{d}}$ of the processes (Fig. 4.16).
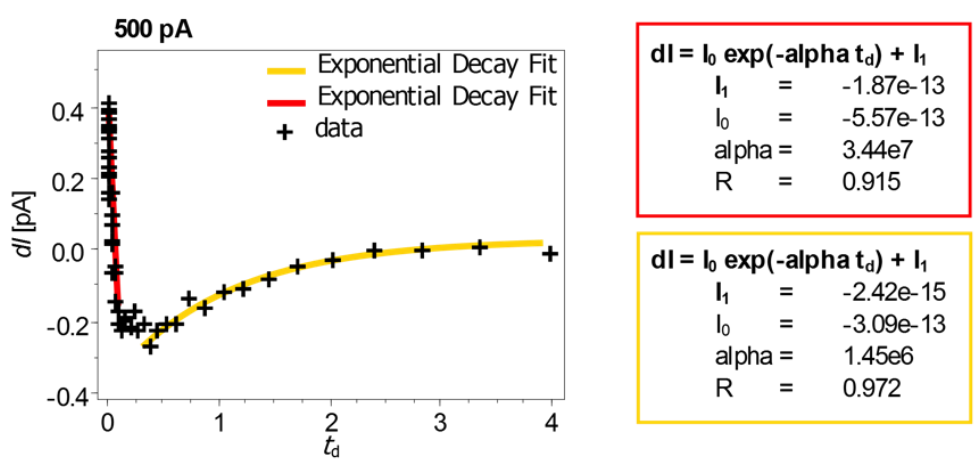

Fig. 4.16 Exemplary fitting results of the $d I\left(t_{\mathrm{d}}\right)$ spectra of Fig. 4.7e in the transition region. In this case the fitting is restricted to early (red) and late (yellow) delay times. The parameter $R$ stands for the nonlinear regression coefficient. The other parameters are self-descriptive. 


\section{S4.6: Logarithmic analysis of the decay spectra of Fig. 4.8b}

In order to ensure the exponential dependency in the spectra in Fig. $\mathbf{4 . 8 b}$, the data is additionally plotted on a logarithmic scale (Fig. 4.17).

For the sake of clarity, the data is plotted another time on normal scale (Fig. 4.17a). Please note that the color assignment is identical to the assignment in the main manuscript. Fig. 4.17b shows the same spectra, plotted on a logarithmic scale. In a first approximation, the assumption of an exponential dependency is sensible.
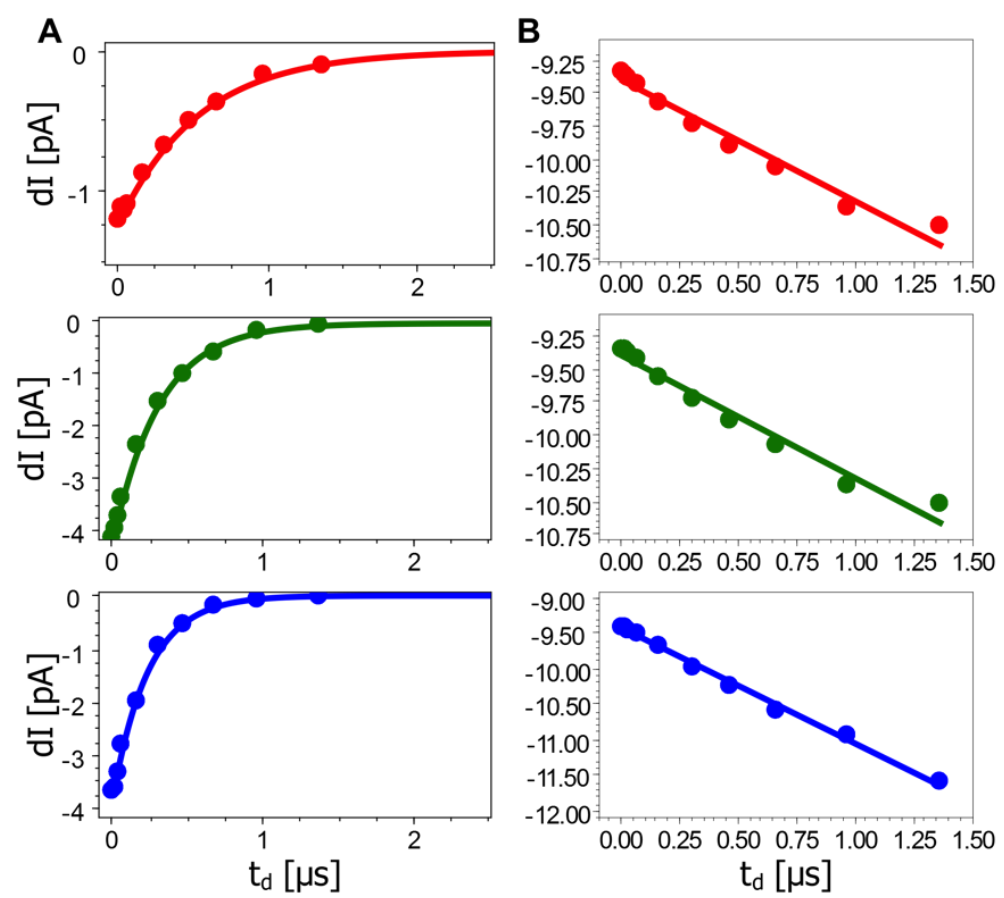

Fig. 4.17 Decay spectra of Fig. 4.8b plotted on normal (a) and logarithmic (b) scale. The color assignment is identical to the main manuscript.

\section{S4.7: Determining the donor depth}

In order to attribute $\tau_{\text {don }}$ to a binding energy in Fig. $\mathbf{4 . 8 d}$, it is necessary to determine the exact depth of the donor. In a first step we order the donors correspondingly to their signature height in the topography (Fig. 4.18a). The surface nearest dopant induces the highest change in the tip height (red dot) whereas the deeper ones show a modulation in tip height, which monotonically decreases (green and then the blue dot) with the depth. For a more exact determination of the donor position we use the stacking sequence of the GaAs atomic layers at the (110) surface. We extract the point of maximum modulation in the topography for each dopant signature and assume that this point is positioned centered above the buried doping atom.

Having an $\mathrm{ABAB}$ stacking sequence at the $\mathrm{GaAs}(110)$ surface this point is either positioned on a corrugation maximum (even) or next to it (odd), i.e. in between two maxima (Fig. 4.18b). With this information we can assign to each donor signature in the topography its position in an even or odd 
layer beneath the surface (Fig. 4.18c). With this information and with the help of comparable, previous studies, performed in our workgroup, we are able to determine the exact depth of the dopant.

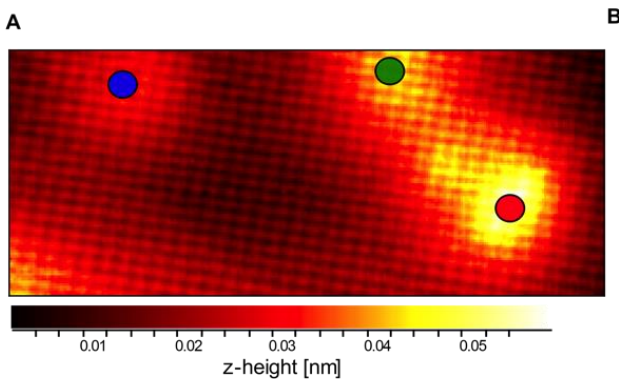

C

surface ০০০০০০০০০০০

layer $10 \bullet 0 \bullet 0 \bullet 000 \bullet 0 \bullet ० \bullet$ Gallium

layer $2 \bullet 0 \bullet 0 \bullet 0 \bigcirc 0 \bullet 0 \bullet 0 \bullet 0$ Arsen

layer $3 \circ \bullet \circ \bullet ० \bullet ० ० ० \bullet ० \bullet ० \bullet$

layer $4 \bullet 0 \bullet 0 \bullet ० ० 0 \bullet ० \bullet ० \bullet$

layer $50 \bullet 0 \bullet 0 \bullet 00000$

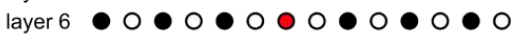

O dopant
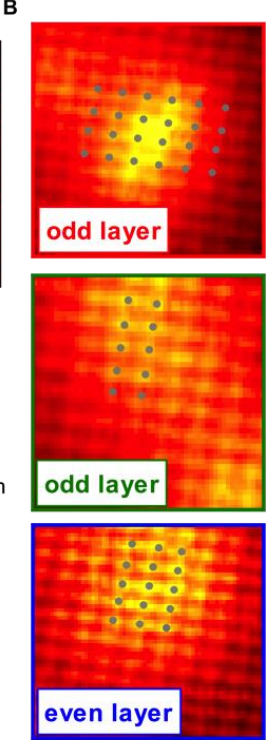

Fig. 4.18 Extracting the dopant depth from STM topographies. (a) STM topography showing the signature of three buried dopants. Depending on the depth, the $z$ height modulation above the dopant decreases. (b) More detailed pictures of each single dopant labeled in red, green and blue in $A$. Grey points trace the atomic corrugation. (c) $A B A B$ stacking at the $\operatorname{GaAs}(110)$ surface. Red dot: layer 1, Green dot: layer 3, Blue dot: layer 4.

\section{S4.8: Temperature dependent analysis of the dopant charging}

Two possible mechanisms for the dopant ionization have been considered to describe the spatial variation of decay time for the electron release from the donor level: thermal emission and fielddriven tunneling of electrons into the conduction band. In order to probe for thermal emission we conducted time spectra at $6 \mathrm{~K}$ (blue line) and $77 \mathrm{~K}$ (red line). Plotted (Fig. 4.19) is the normalized SPPX induced current $d I$ against the delay time $t_{\mathrm{d}}$.

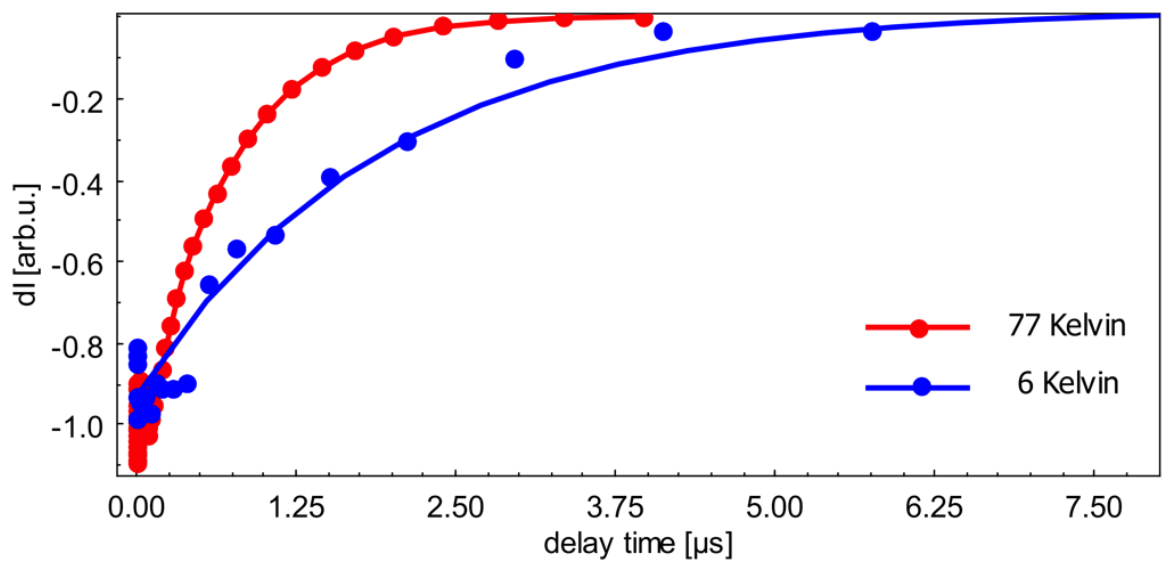

Fig. 4.19 Dopant relaxations, given in $d l$ plotted against the delay time $t_{d}$, for different temperatures. Red line: Decay at $77 \mathrm{~K}$ gives $T_{\text {don }}=711 \mathrm{~ns}(1 \mathrm{~V}$ bias voltage, $1500 \mathrm{pA}$ set point current, exc. parameters: $40 \mathrm{~ns}$ pulse width, $8 \mu \mathrm{s} \mathrm{rep}$. cycle, $8 \mu \mathrm{W}$ average power). Blue line: Decay at $6 \mathrm{~K}$ gives $\tau_{\text {don }}=2 \mu \mathrm{s}(2 \mathrm{~V}$ bias voltage, $400 \mathrm{pA}$ set point current, exc. parameters: $400 \mathrm{~ns}$ pulse width, $32 \mu \mathrm{s}$ rep. cycle, $20 \mu \mathrm{W}$ average power). 
At $77 \mathrm{~K}$ (red line) the dopant decay time $\tau_{\text {don }}(77 \mathrm{~K})$ is given by $711 \mathrm{~ns}$ whereas at $6 \mathrm{~K}$ it takes $\tau_{\text {don }}(6 \mathrm{~K})=2 \mu$ s to relax to the ground state. Theoretically the ratio $R=\tau_{\text {don }}^{T_{1}} / \tau_{\text {don }}^{T_{2}}$ at temperatures $T_{1}$ and $T_{2}$ in case of thermal emission can be estimated by

$$
R=\frac{\tau_{\mathrm{don}}^{T_{1}}}{\tau_{\mathrm{don}}^{T_{2}}}=\frac{\exp \left(-\frac{E_{\mathrm{B}}}{\mathrm{k}_{\mathrm{B}} T_{1}}\right)}{\exp \left(-\frac{E_{\mathrm{B}}}{\mathrm{k}_{\mathrm{B}} T_{2}}\right)}
$$

With a binding energy of $45 \mathrm{meV}$, the ratio $R$ for $T_{1}=77 \mathrm{~K}$ and $T_{2}=6 \mathrm{~K}$ would be large $\left(R \sim 10^{35}\right)$. This contradicts our experimental finding and we conclude that thermal emission is not a relevant process in our case. The difference in the relaxation time $\tau_{\text {dop }}$ in Fig. 4.19 may originate from the different set point or excitation parameters. A further understanding demands for spatially resolved $6 \mathrm{~K}$ measurements.

\section{S4.9: Field-driven tunnel ionization from the donor level into the conduction band}

We propose field-driven tunnel ionization as the relaxation mechanism of an excited, neutral dopants. Fig. 4.20 sketches the situation schematically. The tip-induced potential $\Delta \phi$ at the GaAs surface pulls the conduction band edge below the donor level enabling this process. Two parameters are crucial for the tunneling probability: the electric field $\boldsymbol{E}$ at the donor position and the binding energy $E_{\mathrm{B}}$ of the electron in the donor level.

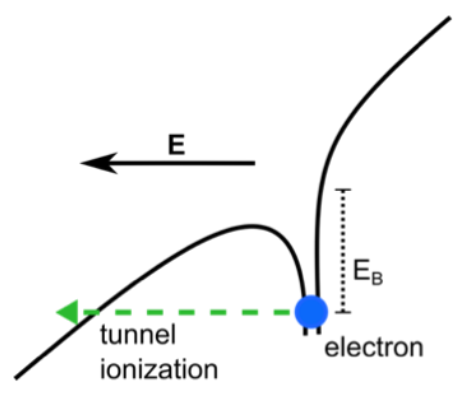

Fig. 4.20 Schematic for field-driven tunnel ionization. The tunneling rate from the donor level into the conduction band is defined by the tip-induced electric field $\boldsymbol{E}$ at the dopant position and the binding energy $E_{\mathrm{B}}$.

As a quantitative model for the charging rate $\omega=\frac{1}{\tau_{\text {hydro }}}$ we use the analytical expression for the ionization process of a hydrogen atom in an electric field $\boldsymbol{E}(25)$ :

$$
\omega=4 \omega_{\mathrm{A}} \frac{E_{\mathrm{A}}}{|\boldsymbol{E}|} \mathrm{e}^{-\frac{2}{3} \frac{E_{\mathrm{A}}}{|E|}}
$$

Adapting this expression to the hydrogen-like donor level of silicon in bulk GaAs the parameters $\omega_{\mathrm{A}}$ and $E_{\mathrm{A}}$ become

$$
\omega_{\mathrm{A}}=\frac{m_{\mathrm{e}} m^{*} e^{4}}{\left(4 \pi \varepsilon_{0} \varepsilon_{\mathrm{r}}\right)^{2} \hbar^{3}}, E_{\mathrm{A}}=\frac{2 m_{\mathrm{e}} m^{*} e^{2} E_{\mathrm{B}}}{4 \pi \varepsilon_{0} \varepsilon_{\mathrm{r}} \hbar^{2}}
$$

with $m^{*}=0.06$ and $\varepsilon_{r}=12.8$. The other notations are standard values. 


\subsection{General discussion}

In a current dependent analysis of time-resolved spectra of the photo-induced signal $d I$ the superposition of two relaxation processes can be determined. One of the processes shows a prominent dependency on the tunneling rate and is therefore attributed to the hole annihilation mechanism via the tunneling into the photo-generated holes. Like already mentioned in chapter 3.3, the recombination of holes via the tunnel current has been widely neglected in former studies. However, in the results of Shigekawa et al. in Fig. 4.3b also a slight correlation between tunnel rate and decay time is observed. According to their model, this is due to tunneling electrons into the conduction band, which recombine with the optically excited holes across the band gap. Though this assumption has its eligibility, there is one major aspect contradicting this prediction. Recent results ${ }^{116}$ have shown very high, ambipolar drift and diffusion rates for charge carriers in GaAs. Consequently it is expected, that electrons, tunneling from the STM tip into the conduction band, will leave the tip-induced space charge region within picoseconds. During this very short time, recombination with holes (characteristic time for this process is in the nanosecond regime) at the surface becomes unlikely. The current dependency of the relaxation rate is more likely to be connected to direct tunneling into the photo-generated charge.

Another prominent feature of our results is the appearance of a second decaying signal at high tunnel currents and especially the sign reversal of $d I$ connected to this. The positive $d I$, detected at low tunneling rates, is due to very low excitation densities (see subsection S4.2), which were never given in the studies of Shigekawa et al.

Nevertheless, two decaying processes were observed ( $\tau_{\mathrm{s}}$ and $\tau_{\mathrm{b}}$ in Fig. 4.3b) as well. Very comparable to our studies, one of the two processes $\left(\tau_{\mathrm{b}}\right)$ shows no current dependency. Shigekawa et al. differentiate between both processes as a surface-site decay $\left(\tau_{\mathrm{s}} \approx 100-200 \mathrm{~ns}\right.$ ) and a bulk-site decay $\left(\tau_{\mathrm{b}} \approx 5 \mathrm{~ns}\right)$ of the photo-excited charge. We have never observed these short decay times. But one has to be aware that in our case, the experiments were conducted at $77 \mathrm{~K}$ or $6 \mathrm{~K}$ whereas all preliminary work was carried out at room temperature. The results in subsection S4.8 indicate a clear decrease in the ionization time of dopants by going from 6 to $77 \mathrm{~K}$. If this trend continues, it will be conceivable that at room temperature the ionization time of the dopants will be as short as the interpreted bulk-side decay in the studies of Shigekawa et al. Eventually, this would imply that their experimental results and ours are not that different but in the end interpreted in completely different manners.

Accessing the ionization process of individual donors reveals a delicate dependency of the decay time on the local atomic configuration. As thermal emission of electrons is ruled out, a field-driven ionization process of the dopants is assumed. Moreover, instead of isolated dopants, they have to be regarded as one stochastically distributed network of defects, where charging events of single network members are coupled electrostatically among each other. 
Shigekawa et al. conducted comparable measurements on deposited Mn atoms at the GaAs surface (Fig. 4.6). But as only single spectra directly above the adatom were taken, information on the local inhomogeneity are missing. Our results demonstrate, for the first time, the dynamics of photogenerated charge on the atomic scale. The requirement of this analysis is nicely confirmed by the varying dynamic behavior of the buried dopants in Fig. 4.8. 



\section{Outlook: The GaAs surface and its perspective}

The outlook discusses the promising future of this powerful technique. The successful implementation of pulsed optical excitation during STM operation has been shown. Nevertheless, many other fascinating subjects are waiting to be treated with this new tool.

Up to here, this thesis treats the charge dynamics inside the tip-induced space charge region (SCR) at the GaAs(110) surface. However, the presented method will have also a significant relevance in more applied systems, e.g., local fields play a crucial role in every semiconductor heterostructure. In the following, two possible examples for future studies will be presented, which were already extensively investigated in our research group.

In the PhD thesis of Karen Teichmann, layered nanostructures were investigated ${ }^{27}$. This includes the GaAs/AlAs/GaAs interface, which is widely used in diode lasers ${ }^{117,118}$. Taken from the $\mathrm{PhD}$ thesis of Karen Teichmann, Fig. 5.1 shows a high-resolution STM topography of this heterostructure ${ }^{27}$.

Fig. 5.1 STM topography across the GaAs/AIAs/GaAs heterostructure. Apart from the bright contrast, induced by the AlAs layer, at both sides of the interface a gradient in the tip height is visible, indicating a space charge region. The bright dots inside the AlAs layer is incorporated InAs. Taken from ${ }^{27}$.

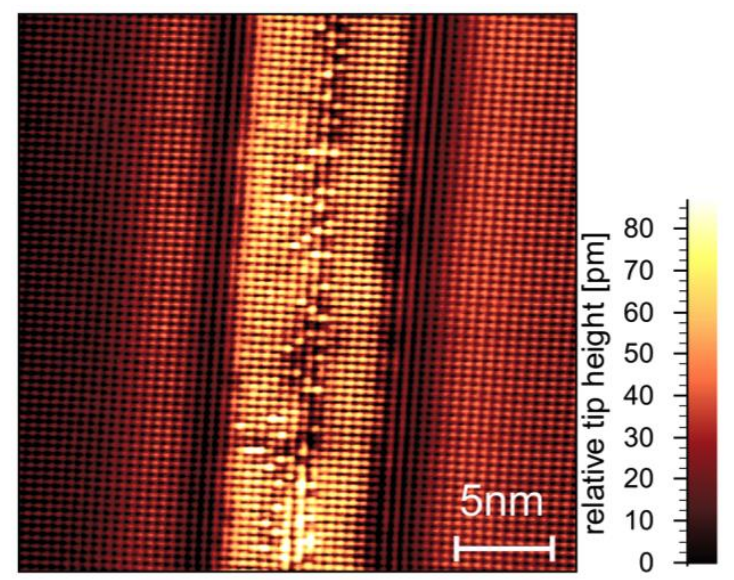

Besides the main feature, given by the $10 \mathrm{~nm}$ thick AlAs interlayer, the contrast is additionally modulated on a longer length scale. This modulation is a direct consequence of a SCR, induced by the interface between GaAs and AlAs. The spatial behavior of this SCR can be better visualized in a spectroscopic section across the layered structure: $I(V)$ curves are laterally resolved (the current is plotted color-coded) with and without optical excitation (Fig. 5.2a, b). 

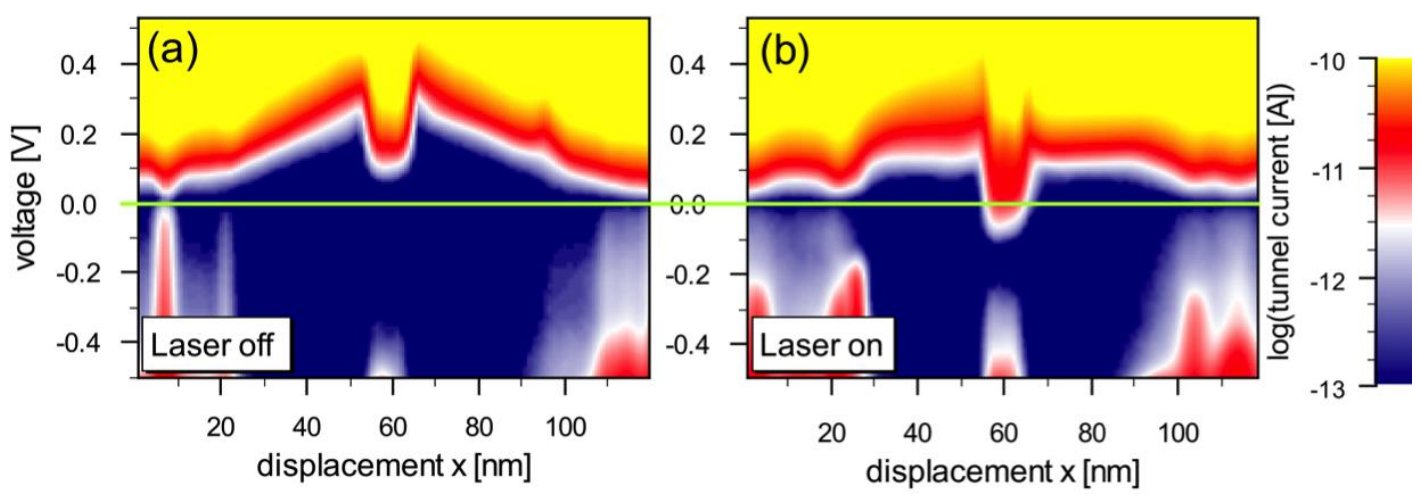

Fig. 5.2 I(V-sections across the GaAs/AIAs/GaAs structure with and without optical excitation. (a) Laser off: The influence of the Al layer is visible as an abrupt decrease in voltage of the conduction band edge in the middle of the section. At both sides of this feature one observes a depleting conduction band edge, showing the spatial extents of the space charge region (SCR). (b) Laser on: By the generation of optically excited, free charge carriers, which are separated in the SCR, force a flattening of the bands. The feature of the AIAs layer moves down in voltage, giving a tunnel current at zero bias voltage. Taken from ${ }^{27}$.

Here, the AlAs layer can be identified as an abrupt decrease of the conduction band edge in the middle of the section. The SCR becomes only visible under dark conditions (Fig. 5.2a). At both sides of the Al layer, the conduction band depletes on a length scale of several tenths of nanometers. Under optical excitation, this changes considerably (Fig. 5.2b). The conduction band flattens. Microscopically, the reason for this behavior is analogue to the SPV for the tip-induced potential: charge carriers are separated inside the SCR and partly accumulate at the GaAs/AlAs junction.

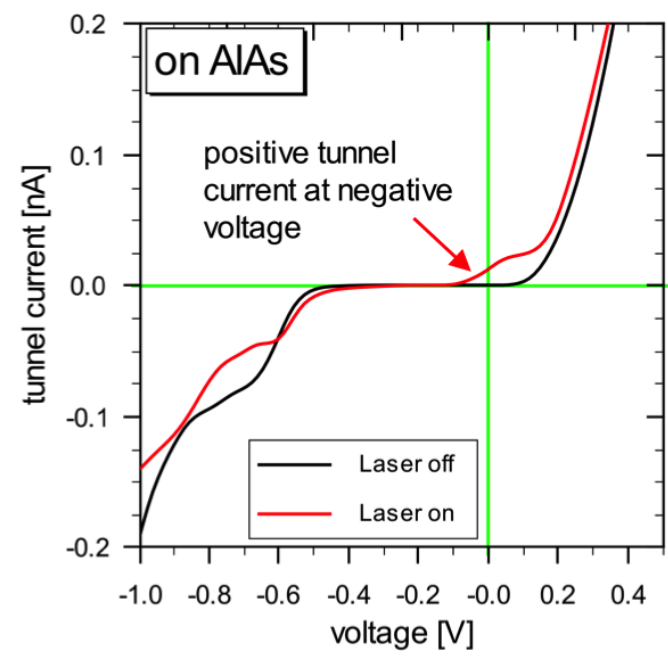

Fig. $5.3 I(V)$ spectra above the AIAs layer with and without optical excitation. In case of optical excitation a zero-bias-voltage tunnel current is obtained. Taken from $^{27}$.

Interestingly, for the AlAs-induced signature in the $I(V)$ spectra (Fig. 5.2b), a tunnel current at zero bias voltage is identified - only present under optical excitation (see also single spectra in Fig. 5.3). In the thesis of Karen Teichmann, this signature is explained with a charge accumulation at the interface in combination with $\Gamma$-X tunneling from the GaAs into the $\mathrm{AlAs}^{27}$. Up to now, a detailed analysis of this interesting phenomenon, widely used in so-called resonant tunneling diodes (RTD), is missing. The optical setup, presented in this thesis, allows, in comparison to the work of Karen Teichmann, the application of very precise laser intensities. By varying the tunneling rate above the 
AlAs layer, one may saturate the $\Gamma-X$ tunneling process and thereby extract its rate. Moreover, with pulsed excitation even the time scales of the charge transport along the RTD might be accessible.

Another possible application is the study of charge dynamics in the SCR of a Schottky contact, which plays a crucial role in the subject of Spintronics. In our research group and in the thesis of Tim Iffländer ${ }^{29,38}$, a Schottky-contact in form of a thin iron (Fe) film on GaAs was prepared and characterized. A double cleavage process inside the UHV gives access to the cross-section along the Fe-GaAs junction (Fig. 5.4).

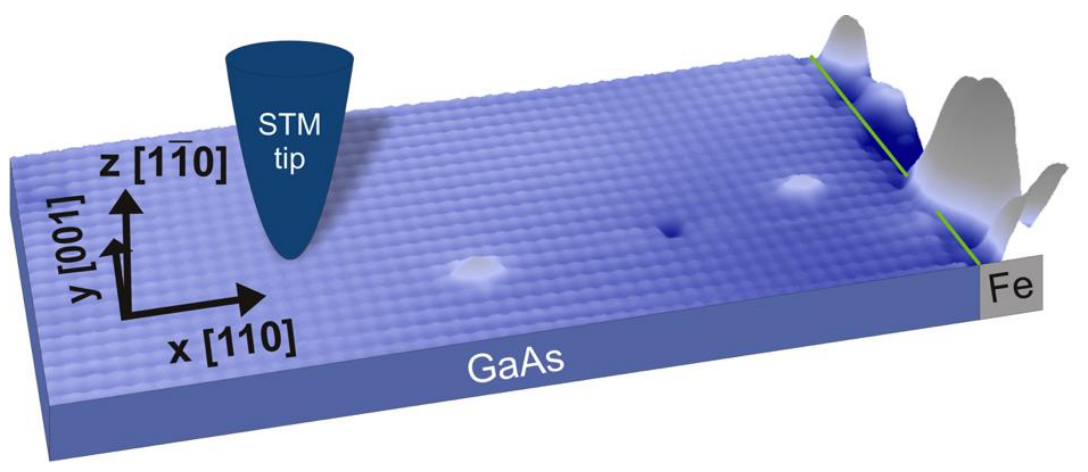

Fig. 5.4 STM topography of the GaAs-Fe interface in the cross-sectional geometry. The green line marks the position of the GaAs surface and thereby the position where the iron film begins. The depletion of the topographic contrast near the junction of both materials indicates yet again a space charge region. Taken from ${ }^{29}$.

The local potential landscape at the Fe-GaAs junction can be directly seen in a spectroscopic crosssection (Fig. 5.5). For this Schottky-contact between Fe and GaAs, charge separates and for pdoped GaAs, the resulting potential will be screened by ionized acceptors. The planar SCR at the side of the GaAs reaches up to $15 \mathrm{~nm}$ into the sample.

Again, the application of laser light at the GaAs surface, results in the generation of electron-hole pairs. Due to the potential landscape at the Schottky-contact, electrons will accumulate in a small region at the junction. Analogue to the GaAs/AlAs interface, the resulting screening configuration between GaAs and Fe will be more efficient and the bands flatten. The concentration of the optically excited and accumulated electrons at the interface and with this the flattening of the bands is defined by a steady state between photo-generation and recombination of charge (chapter 1.2 and Fig. 1.7). The latter one will be mainly given via charge carrier relaxation inside the interface region.

Besides studying the charge dynamics inside this SCR, analogue to chapter 3.3 and 4.4, another possible application may be resolving spin-dependent relaxation mechanism via circularly polarized light. This was already used at the GaAs/AlAs interface for determining spin population asymmetries with an $\mathrm{STM}^{53}$. 


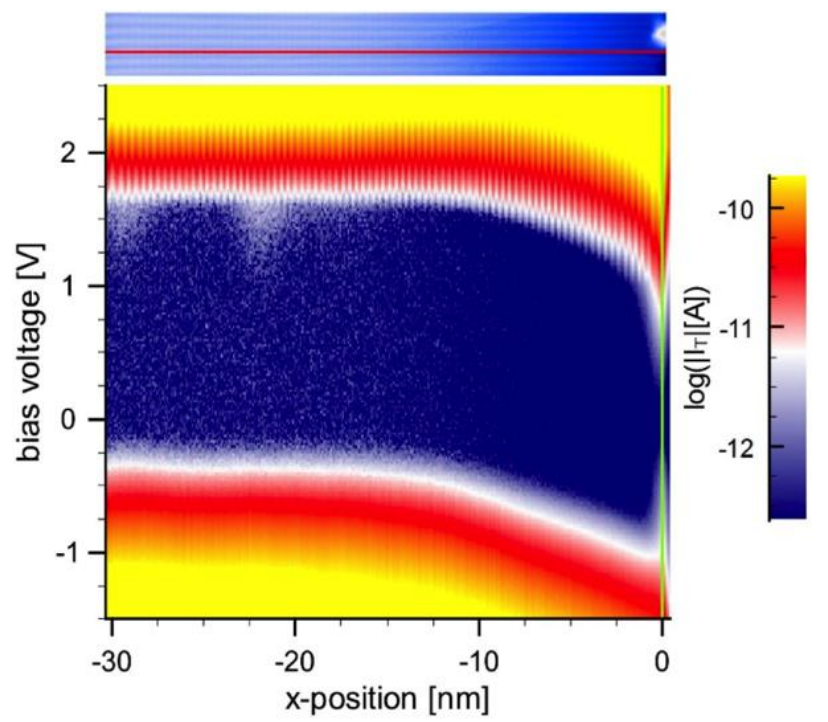

Fig. 5.5 Spectroscopic cross-sections across the Fe-GaAs junction. Tunnel current, plotted color-coded, against the distance to the Fe-GaAs (p-type) junction (green line) and bias voltage. The green line marks the position of $\mathrm{Fe}$ film prepared at the GaAs surface. The planar symmetric space charge region of the Schottky contact extends over $15 \mathrm{~nm}$ into the GaAs surface. Taken from ${ }^{29}$.

In general, circular polarized light will excite spin-polarized electron-hole pairs, which will be separated (completely analogous to the previous paragraph) inside the SCR. This will eventually lead to changing screening configurations at the interface. However, in this case, one has to differentiate between "spin-down" and "spin-up" electrons in order to determine the absolute screening efficiency. Theoretical calculations indicate, that the interface region of the Fe-GaAs junction is spin-polarized ${ }^{29,38}$. Consequently, it can be expected that the rates of the corresponding relaxation channels for the optical-generated charge, accumulated at the interface region, are as well spin-dependent.

In chapter 3.3, the tunnel current is used to modify and control the concentration of photogenerated charge at the surface and thereby used to extract values such as the diffusive or fielddriven capture rate of charge carriers at smooth and defect-free GaAs surfaces. For the magnetic Fe-GaAs Schottky-junction, the application of circularly polarized laser light results in the generation of spin-polarized electron-hole pairs. A similar approach via tuning the tunnel current will allow for determining intrinsic recombination rates with additional spin-resolution at, e.g., interface states (for details on the method see Fig. 3.19). Moreover, by applying pulsed excitation, the dynamical build-up and relaxation of the SCR will become visible. Analog to chapter 4.4, this will provide access to the dynamics of charge recombination processes inside the planar SCR, this time with additional spin resolution.

These two examples demonstrate the promising prospects of this innovative experimental method for further applications. The implementation of time resolution in "nano-sensitive" tools like the STM is at the heartbeat of modern solid-state physics. The access to dynamic processes on the atomic scale opens new pathways for characterizing and improving microscopic structures in a very controlled manner. With regards to our long-time expertise in atomic-scale physics at semiconductor surfaces, this sophisticated technique will take our possibilities and understanding of such systems to the next level. 


\section{References}

${ }^{1}$ P. Peercy, Nature 406, 1023 (2000).

${ }^{2}$ S. Roy and A. Asenov, Science 309, 388 (2005).

${ }^{3}$ C.G.B. Garett and W.H. Brattain, Phys. Rev. 99, (1955).

${ }^{4}$ L. Kronik and Y. Shapira, Surf. Sci. Rep. 37, 1 (1999).

${ }^{5}$ G. Haase, Int. Rev. Phys. Chem. 19, 37 (2000).

${ }^{6}$ G. Binnig, H. Rohrer, C. Gerber, and E. Weibel, Phys. Rev. Lett. 50, 120 (1983).

${ }^{7}$ K. Teichmann, M. Wenderoth, H. Prüser, K. Pierz, H.W. Schumacher, and R.G. Ulbrich, Nano Lett. 13, 3571 (2013).

${ }^{8}$ S. Loth, S. Baumann, C.P. Lutz, D.M. Eigler, and A.J. Heinrich, Science (80-. ). 196, 335 (2012).

${ }^{9}$ H. Pruser, M. Wenderoth, P.P.E. Dargel, A. Weismann, R. Peters, T. Pruschke, R.G. Ulbrich, H. Prüser, M. Wenderoth, and P.P.E. Dargel, Nat Phys 7, 203 (2011).

${ }^{10}$ D.G. Cahill and R.J. Hamers, Phys. Rev. B 44, 1387 (1991).

${ }^{11}$ S. Aloni, I. Nevo, and G. Haase, Phys. Rev. B 60, R2165 (1999).

${ }^{12}$ O. Takeuchi, S. Yoshida, and H. Shigekawa, Appl. Phys. Lett. 84, (2004).

${ }^{13}$ C. Sommerhalter, T.W. Matthes, J. Boneberg, and P. Leiderer, J. Vac. Sci. Technol. B 15, 1876 (1997).

${ }^{14}$ S. Aloni, I. Nevo, and G. Haase, J. Chem. Phys. 115, 1875 (2001).

${ }^{15}$ S. Yoshida, Y. Kanitani, O. Takeuchi, and H. Shigekawa, Appl. Phys. Lett. 92, 102105 (2008).

${ }^{16}$ S. Yoshida, Y. Kanitani, R. Oshima, Y. Okada, O. Takeuchi, and H. Shigekawa, Phys. Rev. Lett. 98, 26802 (2007).

${ }^{17}$ M. Schnedler, V. Portz, P.H. Weidlich, R.E. Dunin-Borkowski, and P. Ebert, Phys. Rev. B 91, 1 (2015).

${ }^{18}$ P. Kloth, K. Kaiser, and M. Wenderoth, Nat. Commun. 7, 10108 (2016).

${ }^{19}$ K. Teichmann, M. Wenderoth, S. Loth, J.K. Garleff, A.P. Wijnheijmer, P.M. Koenraad, and R.G. Ulbrich, Nano Lett. 11, 3538 (2011).

${ }^{20}$ E.P. Smakman, P.L.J. Helgers, J. Verheyen, P.M. Koenraad, and R. Möller, Phys. Rev. B 90, 41410 (2014).

${ }^{21}$ S. Weiss, D.F. Ogletree, D. Botkin, M. Salmeron, and D.S. Chemla, Appl. Phys. Lett. 63, 2567 (1993).

22 S. Grafstrom and S. Grafström, J. Appl. Phys. 91, 1717 (2002). 
${ }^{23}$ R.J. Hamers and D.G. Cahill, Appl. Phys. Lett. 57, 2031 (1990).

${ }^{24}$ O. Takeuchi, M. Aoyama, R. Oshima, Y. Okada, H. Oigawa, N. Sano, H. Shigekawa, R. Morita, and M. Yamashita, Appl. Phys. Lett. 85, 3268 (2004).

${ }^{25}$ Y. Terada, S. Yoshida, O. Takeuchi, and H. Shigekawa, Nat Phot. 4, 869 (2010).

${ }^{26}$ S. Loth, Atomic Scale Images of Acceptors in III-V Semiconductors: Band Bending, Tunneling Paths and Wave Functions, Georg-August-Universität Göttingen, 2007.

${ }^{27}$ K. Teichmann, Scanning Tunneling Spectroscopy of Space Charge Regions in Semiconductors : From Single Donor to Heterostructure Systems Karen Teichmann, Georg-August-Universität Göttingen, 2012.

${ }^{28}$ H. Prüser, Scanning Tunneling Spectroscopy of Magnetic Bulk Impurities, Springer International Publishing, 2015.

${ }^{29}$ T. Iffländer, Electronic and Magnetic Properties of the Fe / GaAs ( 110 ) Interface, GeorgAugust-Universität Göttingen, 2015.

${ }^{30}$ G. Münnich, Cross-Sectional Scanning Probe Microscopy on GaAs : Tip-Induced Band Bending , Buried Acceptors and Adsorbed Molecules Dissertation, University of Regengsburg, 2014.

${ }^{31}$ S.Y. Tong, A.R. Lubinsky, B.J. Mrstik, and M.A. Van Hove, Phys. Rev. B 17, 3303 (1978).

${ }^{32}$ K.P. O’Donnell and X. Chen, Appl. Phys. Lett. 58, 2924 (1991).

${ }^{33}$ P. Ebert, B. Engels, P. Richard, K. Schroeder, S. Blügel, C. Domke, M. Heinrich, and K. Urban, Phys. Rev. Lett. 77, 2997 (1996).

${ }^{34}$ J.J.R. Chelikowsky and M.M.L. Cohen, Phys. Rev. B 20, 4150 (1979).

${ }^{35}$ R.M. Feenstra, J. A., J. Tersoff, and A.P. Fein, Phys. Rev. Lett. 58, (1987).

${ }^{36}$ M.A. Rosentreter, M. Wenderoth, N.H. Theuerkrauf, A.J. Heinrich, M.A. Schneider, and R.G. Ulbrich, Phys. Rev. B 56, 10538 (1997).

${ }^{37}$ K. Sauthoff, M. Wenderoth, A.J. Heinrich, M.A. Rosentreter, K.J. Engel, T.C.G. Reusch, and R.G. Ulbrich, Phys. Rev. B 60, 4789 (1999).

38 T. Iffländer, S. Rolf-Pissarczyk, L. Winking, R.G. Ulbrich, A. Al-Zubi, S. Blügel, and M. Wenderoth, Phys. Rev. Lett. 114, 146804 (2015).

${ }^{39}$ R.M. Feenstra, J. Vac. Sci. Technol. B Microelectron. Nanom. Struct. 5, 923 (1987).

${ }^{40}$ R.M. Feenstra, G. Meyer, F. Moresco, and K.H. Rieder, Phys. Rev. B 66, 165204 (2002).

${ }^{41}$ R. Dombrowski, C. Steinebach, C. Wittneven, M. Morgenstern, and R. Wiesendanger, Phys. Rev. B 59, 8043 (1999).

${ }^{42}$ C. Garrett and W. Brattain, Phys. Rev. 99, 376 (1955).

${ }^{43}$ W.H. Brattain and C.G.B. Garrett, Bell Syst. Tech. J. 35, 1019 (1956).

${ }^{44}$ A.M. Goodman, J. Appl. Phys. 32, 2550 (1961).

${ }^{45}$ R.M. Feenstra, J. Vac. Sci. Technol. B Microelectron. Nanom. Struct. 5, 923 (1987). 
${ }^{46}$ M. Hecht, Phys. Rev. B 41, 7918 (1990).

${ }^{47}$ D. Schroder, Meas. Sci. Technol. 16, (2001).

${ }^{48}$ G. Nunes and M.R. Freeman, Science 262, 1029 (1993).

${ }^{49}$ D. Botkin, J. Glass, D.S. Chemla, D.F. Ogletree, M. Salmeron, and S. Weiss, Appl. Phys. Lett. 69, 1321 (1996).

${ }^{50}$ R. Hamers and D. Cahill, Appl. Phys. Lett. 2031, 1 (1990).

${ }^{51}$ S. Grafström, P. Schuller, J. Kowalski, and R. Neumann, J. Appl. Phys. 83, 3453 (1998).

${ }^{52}$ Y. Terada, S. Yoshida, O. Takeuchi, and H. Shigekawa, Nat. Photonics 4, 869 (2010).

${ }^{53}$ S. Yoshida, Y. Aizawa, Z. Wang, R. Oshima, Y. Mera, E. Matsuyama, H. Oigawa, O. Takeuchi, and H. Shigekawa, Nat. Nanotechnol. 9, 588 (2014).

${ }^{54}$ S. Yoshida and Y. Terada, Appl. Phys. ... 6, 1 (2013).

${ }^{55}$ S. Loth, M. Etzkorn, C.P. Lutz, D.M. Eigler, and A.J. Heinrich, Science (80-. ). 329, 1628 (2010).

${ }^{56}$ C. Saunus, J. Raphael Bindel, M. Pratzer, and M. Morgenstern, Appl. Phys. Lett. 102, (2013).

${ }^{57}$ C. Grosse, M. Etzkorn, K. Kuhnke, S. Loth, and K. Kern, Appl. Phys. Lett. 103, (2013).

${ }^{58}$ T. Cocker, V. Jelic, and M. Gupta, Nat. Photonics 7, 620 (2013).

${ }^{59}$ T.L. Cocker, D. Peller, P. Yu, J. Repp, and R. Huber, Nature 539, 263 (2016).

${ }^{60}$ R.J. Hamers and K. Markert, Phys. Rev. Lett. 64, 1051 (1990).

${ }^{61}$ C.C. Williams, W.P. Hough, and S.A. Rishton, Appl. Phys. Lett. 55, 203 (1989).

${ }^{62}$ V. Gerstner, A. Knoll, W. Pfeiffer, A. Thon, and G. Gerber, J. Appl. Phys. 88, (2000).

${ }^{63}$ S. Yoshida, Y. Terada, R. Oshima, O. Takeuchi, and H. Shigekawa, Nanoscale 4, 757 (2012).

${ }^{64}$ S. Yoshida, M. Yokota, O. Takeuchi, H. Oigawa, Y. Mera, and H. Shigekawa, Appl. Phys. Express 6, 32401 (2013).

${ }^{65}$ A. Dolocan, D.P. Acharya, P. Zahl, P. Sutter, and N. Camillone, J. Phys. Chem. C 115, 10033 (2011).

${ }^{66}$ L.Q. Qian and B.W. Wessels, Appl. Phys. Lett. 1295, 23 (1991).

${ }^{67}$ S. Akari, M.C. Lux- Steiner, M. Vögt, M. Stachel, and K. Dransfeld, J. Vac. Sci. \&amp; Technol. B 9, 561 (1991).

${ }^{68}$ C. Sommerhalter and T. Matthes, J. Vac. ... 1876, (1997).

${ }^{69}$ R.J. Hamers and D.G. Cahill, J. Vac. Sci. Technol. B 9, (1990).

${ }^{70}$ N.N. Khusnatdinov, T.J. Nagle, and J. G. Nunes, Appl. Phys. Lett. 77, (2000).

${ }^{71}$ S.W. Wu and W. Ho, Phys. Rev. B 82, 85444 (2010). 
${ }^{72}$ H. Shigekawa, O. Takeuchi, and M. Aoyama, Sci. Technol. Adv. Mater. 6, 582 (2005).

${ }^{73}$ N. Xie, H. Gong, S. Yan, J. Zhao, X. Shan, Y. Guo, Q. Sun, and X. Lu, Appl. Phys. Lett. 213104, (2012).

${ }^{74}$ J. Snoddy, Y. Li, F. Ravet, and X. Bao, Appl. Opt. 46, 1482 (2007).

${ }^{75}$ B. Wessels and L. Qian, J. Vac. Sci. Technol. ... 1803, 1 (1992).

${ }^{76}$ T. Takahashi and M. Yoshita, Appl. Phys. Lett. 68, 3479 (1996).

${ }^{77}$ T. Takahashi and M. Masahiro, Appl. Phys. Lett. 70, 2162 (1997).

${ }^{78}$ S. Weiss, D. Botkin, D.F. Ogletree, M. Salmerón, and D.S. Chemla, Phys. Status Solidi B 188, (1995).

${ }^{79}$ D.G. Cahill and R.M. Feenstra, J. Vac. Sci. Technol. A Vacuum, Surfaces, Film. 11, 792 (1993).

${ }^{80}$ D.G. Cahill and R.J. Hamers, J. Vac. Sci. \&amp; Technol. B 9, 564 (1991).

${ }^{81}$ M. McEllistrem, G. Haase, D. Chen, and R.J. Hamers, Phys. Rev. Lett. 70, 2471 (1993).

${ }^{82}$ K. Sell, I. Barke, S. Polei, C. Schumann, V. von Oeynhausen, and K.-H. Meiwes-Broer, Phys. Status Solidi n/a (2010).

${ }^{83}$ F.R.F. Fan and A.J. Bard, J. Phys. Chem. 97, 1431 (1993).

${ }^{84}$ K.S. Ralls, W.J. Skocpol, L.D. Jackel, R.E. Howard, L.A. Fetter, R.W. Epworth, and D.M. Tennant, Phys. Rev. Lett. 52, 228 (1984).

${ }^{85}$ D.H. Lee and J.A. Gupta, Science (80-. ). 330, 1807 (2010).

${ }^{86}$ P.M. Koenraad and M.E. Flatté, Nat. Mater. 10, 91 (2011).

${ }^{87}$ K. Teichmann, M. Wenderoth, S. Loth, R.G. Ulbrich, J.K. Garleff, A.P. Wijnheijmer, and P.M. Koenraad, Phys. Rev. Lett. 101, 76103 (2008).

${ }^{88}$ R.M. Feenstra and J.A. Stroscio, J. Vac. Sci. Technol. B 923, (5AD).

${ }^{89}$ H. Zheng, A. Weismann, and R. Berndt, Nat. Commun. 5, 1 (2014).

${ }^{90}$ I. Fernández-Torrente, D. Kreikemeyer-Lorenzo, a. Stróżecka, K.J. Franke, and J.I. Pascual, Phys. Rev. Lett. 108, 36801 (2012).

${ }^{91}$ E.P. Smakman, P.L.J. Helgers, J. Verheyen, P.M. Koenraad, and R. Möller, Phys. Rev. B 90, 41410 (2014).

${ }^{92}$ B.W. Heinrich, L. Braun, J.I. Pascual, and K.J. Franke, Nat Phys 9, 765 (2013).

${ }^{93}$ T. Chen, S. Fung, and C. Beling, Solid State Commun. 89, 779 (1994).

${ }^{94}$ G. Binnig and H. Rohrer, Surf. Sci. 126, 236 (1983).

${ }^{95}$ M.D. Sturge, Phys. Rev. 127, 768 (1962).

${ }^{96}$ E.P. Smakman, J. van Bree, and P.M. Koenraad, Phys. Rev. B 87, 85414 (2013). 
${ }^{97}$ N.L. Schneider, G. Schull, and R. Berndt, Phys. Rev. Lett. 105, 26601 (2010).

${ }^{98}$ F. Pulizzi, A.J. Kent, A. Patanè, L. Eaves, and M. Henini, Appl. Phys. Lett. 84, 3046 (2004).

${ }^{99}$ L. Kronik and Y. Shapira, Surf. Interface Anal. 31, 954 (2001).

${ }^{100}$ N. Puhlmann, G. Oelgart, V. Gottschalch, and R. Nemitz, Semicond. Sci. Technol. 6, 181 (1991).

${ }^{101}$ A.P. Wijnheijmer, K. Teichmann, M. Wenderoth, S. Loth, R.G. Ulbrich, P.A. Maksym, M. Roy, and P.M. Koenraad, Phys. Rev. Lett. 102, 166101 (2009).

102 P.T. Landsberg, Recombination in Semiconductors (Cambridge Universitiy Press, Cambridge, 1991).

103 J. Shah, Ultrafast Spectroscopy of Semiconductors and Semiconductor Nanostructures (Springer, New York, 1996).

${ }^{104}$ Y. Yang, J. Gu, J.L. Young, E.M. Miller, J.A. Turner, N.R. Neale, and M.C. Beard, Science (80. ). 350, 1061 (2015).

${ }^{105}$ G.P. Lansbergen, Nat. Nanotechnol. 7, 209 (2012).

${ }^{106}$ M.M. Waldrop, Nature 530, 144 (2016).

${ }^{107}$ R.M. Feenstra, Phys. Rev. B 50, 4561 (1994).

${ }^{108}$ O.B. Aphek, L. Kronik, M. Leibovitch, and Y. Shapira, Surf. Sci. 409, 485 (1998).

109 A.P. Wijnheijmer, J.K. Garleff, K. Teichmann, M. Wenderoth, S. Loth, R.G. Ulbrich, P.A. Maksym, M. Roy, and P.M. Koenraad, Phys. Rev. Lett. 102, 166101 (2009).

${ }^{110}$ E.L. L. Landau, in Quantum Mech. (Pergamon, New York, 1965).

${ }^{111}$ J. K. Garleff, A. P. Wijnheijmer, C. N. v. d.Enden, and P. M. Koenraad, Phys. Rev. B 84, 75459 (2011).

${ }^{112}$ Y.M. Blanter and M. Büttiker, Phys. Rep. 336, 1 (2000).

${ }^{113}$ T.H. Oosterkamp, T. Fujisawa, W.G. van der Wiel, K. Ishibashi, R. V. Hijman, S. Tarucha, and L.P. Kouwenhoven, Nature 395, 873 (1998).

${ }^{114}$ L.C.L. Hollenberg, a. S. Dzurak, C. Wellard, a. R. Hamilton, D.J. Reilly, G.J. Milburn, and R.G. Clark, Phys. Rev. B 69, 5 (2003).

115 J. Salfi, J.A. Mol, R. Rahman, G. Klimeck, M.Y. Simmons, L.C.L. Hollenberg, and S. Rogge, Nat. Mater. 13, 605 (2014).

${ }^{116}$ B.A. Ruzicka, L.K. Werake, H. Samassekou, and H. Zhao, Appl. Phys. Lett. 97, 262119 (2010).

${ }^{117}$ M.L. Dotor, M. Recio, D. Golmayo, and F. Briones, J. Appl. Phys. 72, 5861 (1992).

${ }^{118}$ L. Schrottke, X. L??, G. Rozas, K. Biermann, and H.T. Grahn, Appl. Phys. Lett. 108, (2016).

119 O. Bunjes, Thermische Effekte Bei Optischer Anregung Im Rastertunnelmikroskop Thermal Processes due to Optical Excitation in a Scanning Tunnelling Microscope, Georg-AugustUniversity Göttingen, 2014. 